\title{
GLOBAL ANALYSIS OF QUASILINEAR WAVE EQUATIONS ON ASYMPTOTICALLY KERR-DE SITTER SPACES
}

\author{
PETER HINTZ AND ANDRAS VASY
}

\begin{abstract}
We consider quasilinear wave equations on manifolds for which infinity has a structure generalizing that of Kerr-de Sitter space; in particular the trapped geodesics form a normally hyperbolic invariant manifold. We prove the global existence and decay, to constants for the actual wave equation, of solutions. The key new ingredient compared to earlier work by the authors in the semilinear case [33] and by the first author in the non-trapping quasilinear case [30] is the use of the Nash-Moser iteration in our framework.
\end{abstract}

\section{INTRODUCTION}

We consider quasilinear wave equations on manifolds for which infinity has a structure generalizing that of Kerr-de Sitter space. An important feature is that, as in perturbations of Kerr-de Sitter space, the trapped geodesics form a normally hyperbolic invariant manifold. We prove the global existence and decay of solutions; this means decay to constants for the actual wave equation. This result is part of a new framework for solving quasilinear wave equations with normally hyperbolic trapping, which extends the semilinear framework developed by the two authors [33] and the non-trapping quasilinear theory developed by the first author [30]. The main new tool introduced here is a Nash-Moser iteration necessitated by the loss of derivatives in the linear estimates at the normally hyperbolic trapping. To our knowledge, this is the first global result for the forward problem for a quasilinear wave equation on either a Kerr or a Kerr-de Sitter background. We remark, however, that Dafermos, Holzegel and Rodnianski [9] have constructed backward solutions for Einstein's equations on the Kerr background; for backward constructions the trapping does not cause difficulties. For concreteness, we state our results first in the special case of Kerr-de Sitter space, but it is important to keep in mind that the setting is more general.

The region of Kerr-de Sitter space we are interested in is a (non-compact) 4dimensional manifold

$$
M^{\circ}=\mathbb{R}_{t_{*}} \times\left(r_{-}-2 \delta, r_{+}+2 \delta\right)_{r} \times \mathbb{S}^{2},
$$

which extends past the event horizon (at $r=r_{-}$) and past the cosmological horizon (at $r=r_{+}$). The manifold $M^{\circ}$ is equipped with a stationary Lorentzian metric

Date: April 4, 2014. Final revision: July 12, 2015.

1991 Mathematics Subject Classification. 35L72, 35L05, 35P25.

Key words and phrases. Quasilinear waves, Kerr-de Sitter space, b-pseudodifferential operators, Nash-Moser iteration, resonances, asymptotic expansion.

The authors were supported in part by A.V.'s National Science Foundation grants DMS0801226 and DMS-1068742 and P.H. was supported in part by a Gerhard Casper Stanford Graduate Fellowship. 
$g_{0}$ (i.e. $\partial_{t_{*}}$ is a Killing vector field) which depends on three parameters, $\Lambda>0$ (the cosmological constant), $M_{\bullet}>0$ (the black hole mass) and $a$ (the angular momentum), though we usually drop this in the notation. We will always assume that $\Lambda, M_{\bullet}$ and $a$ are such that the non-degeneracy condition [51, (6.2)] holds, which in particular ensures that the cosmological horizon lies outside the black hole event horizon, i.e. $r_{+}>r_{-}$. Concretely, in Boyer-Lindquist coordinates $(t, r, \theta, \phi)$ on $\mathbb{R}_{t} \times\left(r_{-}, r_{+}\right)_{r} \times \mathbb{S}^{2}$, the Kerr-de Sitter metric, see e.g. [51, Equation (6.1)], ${ }^{1}$ does not extend to $r=r_{ \pm}$due to a coordinate singularity, but introducing $t_{*}=t+h(r)$, $\phi_{*}=\phi+P(r)$ with suitable functions $h, P$ as in [51, Equation (6.5)], the metric does extend smoothly to $r=r_{ \pm}$and beyond.

In order to set up our problem, see Figure 1 for an illustration, we consider the domain

$$
\Omega^{\circ}=[0, \infty)_{t_{*}} \times\left[r_{-}-\delta, r_{+}+\delta\right]_{r} \times \mathbb{S}^{2} \subset M^{\circ},
$$

which is a submanifold with corners with two boundary hypersurfaces, which are the intersections of

$$
H_{1}=\left\{t_{*}=0\right\}, \quad H_{2}=\left\{r=r_{-}-\delta\right\} \cup\left\{r=r_{+}+\delta\right\}
$$

with $\Omega^{\circ}$. Thus, $H_{1}$ is a Cauchy hypersurface, and $H_{2}$ has two connected components, both spacelike hypersurfaces. We are interested in solving the forward problem for wave-like equations in $\Omega^{\circ}$, i.e. imposing vanishing Cauchy data at $H_{1}$; initial value problems with general Cauchy data can always be converted into an equation of this type.

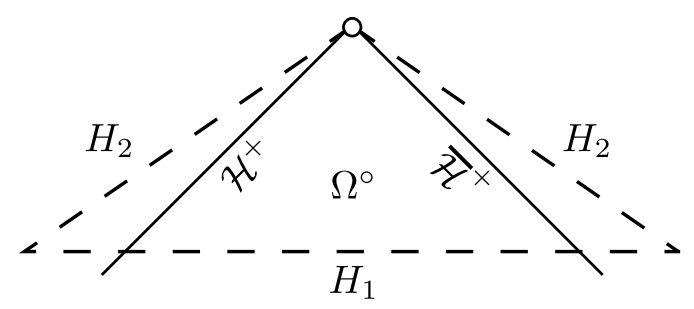

Figure 1. Penrose diagram of the domain $\Omega^{\circ}$, bounded by the dashed lines, on which we solve quasilinear wave equations. $\mathcal{H}^{+}$ is the event horizon, $\overline{\mathcal{H}}^{+}$the cosmological horizon, further $H_{1}$ is a Cauchy hypersurface, where we impose vanishing Cauchy data, and $H_{2}$ has two spacelike connected components.

The simplest (albeit not the most natural from a geometric perspective, see below) wave equations we consider are of the form

$$
\square_{g(u, d u)} u=f+q(u, d u),
$$

$u$ real-valued, where $g(0,0)=g_{0}$ is the Kerr-de Sitter metric, and at each $p=$ $\left(t_{*}, r, \omega\right) \in M^{\circ}$, the metric $g(u, d u)$ is $g_{p}(u(p), d u(p))$, where

$$
g_{p}: \mathbb{R} \oplus T_{p}^{*} M \rightarrow S^{2} T_{p}^{*} M
$$

\footnotetext{
${ }^{1}$ Our $t, \phi, t_{*}, \phi_{*}$ are denoted $\widetilde{t}, \widetilde{\phi}, t, \phi$ in [51].
} 
depends smoothly on $p$, and in fact only depends on $(r, \omega)$, but not on $t_{*}$; further

$$
q(u, d u)=\sum_{j=1}^{N^{\prime}} a_{j} u^{e_{j}} \prod_{k=1}^{N_{j}} X_{j k} u, \quad e_{j}, N_{j} \in \mathbb{N}_{0}, N_{j}+e_{j} \geq 2,
$$

with $a_{j} \in \mathcal{C}^{\infty}\left(M^{\circ}\right)$ and $X_{j k} \in \mathcal{V}\left(M^{\circ}\right)$ real and independent of $t_{*}$. (Here $a_{j}$ is only relevant if $N_{j}=0$, since it can otherwise be absorbed into $X_{j 1}$.)

Using the stationary nature of the spacetime further, we will use the Sobolev spaces $H^{s}$ on $M^{\circ}=\mathbb{R}_{t_{*}} \times X$, with $X=\left(r_{-}-2 \delta, r_{+}+2 \delta\right)_{r} \times \mathbb{S}^{2}$ considered as an open subset of $\mathbb{R}_{y}^{3}$; thus, the norm of $u \in H^{s}$ is defined by

$$
\|u\|_{H^{s}}^{2}=\sum_{j+|\beta| \leq s}\left\|\partial_{t_{*}}^{j} \partial_{y}^{\beta} u\right\|_{L^{2}\left(M^{\circ},\left|d g_{0}\right|\right)}^{2}
$$

Our central result in the form which is easiest to state is:

Theorem 1. For $g(0,0)=g_{0}$ a Kerr-de Sitter metric with angular momentum $|a| \ll M_{\bullet}$ as above, and for $\alpha>0$ sufficiently small and $f \in \mathcal{C}_{\mathrm{c}}^{\infty}\left(M^{\circ}\right)$ real-valued with sufficiently small $H^{12}$-norm, the wave equation $\square_{g(u, d u)} u=f+q(u, d u)$ in $\Omega^{\circ}$, with vanishing Cauchy data, and with $q$ as above with $N_{j} \geq 1$ for all $j$, has a unique real smooth (in $\Omega^{\circ}$ ) global forward solution of the form $u=u_{0}+\widetilde{u}, e^{\alpha t_{*}} \widetilde{u}$ bounded, $u_{0}=c \chi, c \in \mathbb{R}$, where $\chi=\chi\left(t_{*}\right)$ is identically 1 for large $t_{*}$. More precisely, for any fixed $s \in \mathbb{R}$, we will have $e^{\alpha t_{*}} \widetilde{u} \in H^{s}$ if $f$ is sufficiently small (depending on $s)$.

Further, the analogous conclusion holds for the Klein-Gordon operator $\square-m^{2}$ with $m>0$ sufficiently small, without the presence of the $u_{0}$ term, i.e. for $\alpha>$ $0, m>0$ sufficiently small, if $f \in \mathcal{C}_{\mathrm{c}}^{\infty}\left(\Omega^{\circ}\right)$ has sufficiently small $H^{12}$-norm, $\left(\square_{g(u, d u)}-m^{2}\right) u=f+q(u, d u)$ has a unique smooth global forward solution $u \in e^{-\alpha t_{*}} L^{\infty}\left(\Omega^{\circ}\right)$. In fact, for Klein-Gordon equations one can also obtain a leading term, analogously to $u_{0}$, which now has the form $c e^{-i \sigma_{1} t_{*}} \chi, \sigma_{1}$ the resonance of $\square_{g(0)}-m^{2}$ with the largest imaginary part; thus $\operatorname{Im} \sigma_{1}<0$, so this is a decaying solution.

The only reason the assumption $|a| \ll M_{\bullet}$ is made is due to the possible presence (to the extent that we do not disprove it here) of resonances in $\operatorname{Im} \sigma \geq 0$, apart from the 0 -resonance with constants as the resonant state, for larger $a$. Below, in $\S 2$, we give a general result in a form that makes it clear that this is the only remaining item to check - indeed, this even holds in natural vector bundle settings.

We proceed to describe the natural geometric setup in which our analysis of quasilinear wave equations will take place. To begin with, one can conveniently encode the uniform structure of $\left(M^{\circ}, g_{0}\right)$ as $t_{*} \rightarrow \infty$ by adding an 'ideal boundary' at infinity, that is, by introducing

$$
x:=e^{-t_{*}}
$$

and adding $x=0$ to the spacetime, obtaining the 4 -dimensional manifold

$$
M=[0, \infty)_{x} \times\left(r_{-}-2 \delta, r_{+}+2 \delta\right)_{r} \times \mathbb{S}^{2}
$$

with boundary $X=\{x=0\}$. The Lorentzian metric $g_{0}$ then has a specific structure at $\partial M$, i.e. 'infinity', called a totally characteristic, or b-, structure, which we recall below. The closure of $\Omega^{\circ}$ in $M$ is the compact domain $\Omega=[0,1]_{x} \times\left[r_{-}-\delta, r_{+}+\right.$ $\delta]_{r} \times \mathbb{S}^{2}$, which is a submanifold with corners with three boundary hypersurfaces which are the intersections of $H_{1}, H_{2}$ (defined above) and $\partial M$ (the boundary at 
future infinity) with $\Omega$; see Figure 2. We are interested in solving forward problems for wave-like equations in $\Omega$ with vanishing Cauchy data at $H_{1}$ as above.

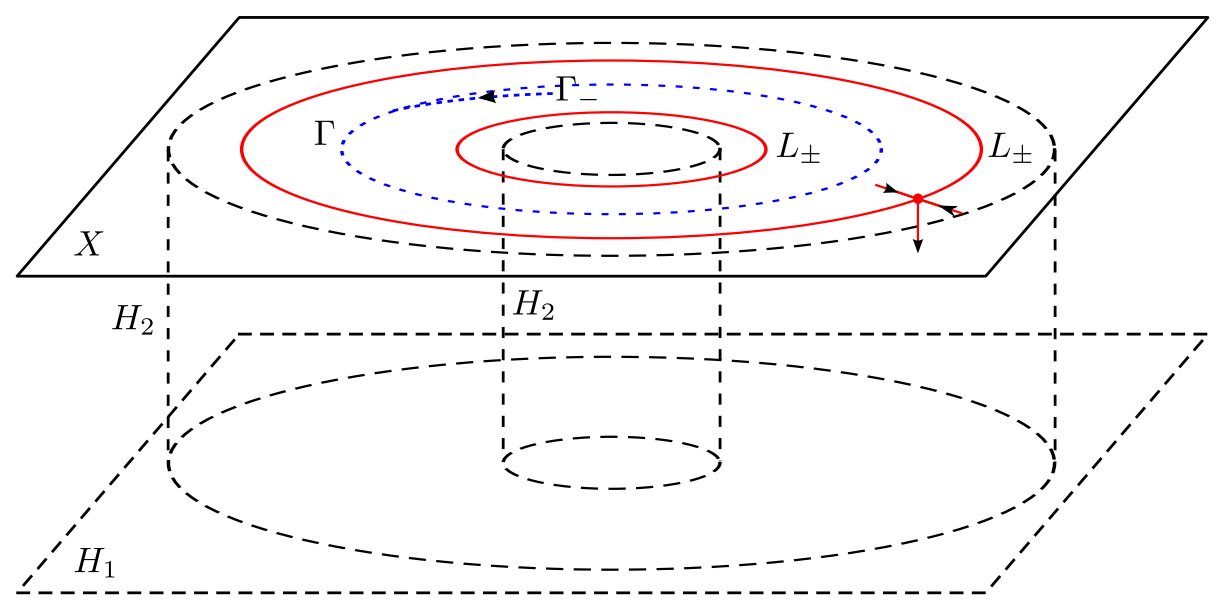

Figure 2. Setup for the discussion of the forward problem on Kerr-de Sitter space from a compactified perspective. Indicated are the ideal boundary $X$, the Cauchy hypersurface $H_{1}$ and the hypersurface $H_{2}$, which has two connected components which lie beyond the cosmological horizon and beyond the black hole event horizon, respectively. The domain $\Omega$ is bounded by $H_{1}$ in the past and by $X$ and $H_{2}$ in the future. The horizons at $X$ themselves are the projections to the base of the (generalized) radial sets $L_{ \pm}$, discussed below, each of which has two components, corresponding to the two horizons. The projection to the base of the bicharacteristic flow is indicated near a point on $L_{+}$; near $L_{-}$, the directions of the flowlines are reversed. Lastly, $\Gamma$ is the trapped set, and the projection of a trapped trajectory approaching $\Gamma$ within $\Gamma_{-}=\Gamma_{-}^{+} \cup \Gamma_{-}^{-}$, discussed below, is indicated.

Now, recall that on any $n$-dimensional manifold with boundary $M$, the Lie algebra of smooth vector fields tangent to the boundary is denoted by $\mathcal{V}_{\mathrm{b}}(M)$; in local coordinates $\left(x, y_{1}, \ldots, y_{n-1}\right)$, with $x$ a boundary defining function, these are linear combinations of $x \partial_{x}$ and $\partial_{y_{j}}$ with $\mathcal{C}^{\infty}(M)$ coefficients. Just as a dual metric is a linear combination of symmetric tensor products of coordinate vector fields, a dual metric in this totally characteristic setting, also called a dual b-metric, is a linear combination of

$$
x \partial_{x} \otimes x \partial_{x}, \frac{1}{2}\left(x \partial_{x} \otimes \partial_{y_{j}}+\partial_{y_{j}} \otimes x \partial_{x}\right), \frac{1}{2}\left(\partial_{y_{i}} \otimes \partial_{y_{j}}+\partial_{y_{j}} \otimes \partial_{y_{i}}\right) .
$$

One can think of this as a symmetric bilinear form; then a Lorentzian dual b-metric is a non-degenerate bilinear form of signature $(1, n-1)$. The actual metric is then a linear combination of

$$
\frac{d x}{x} \otimes \frac{d x}{x}, \frac{1}{2}\left(\frac{d x}{x} \otimes d y_{j}+d y_{j} \otimes \frac{d x}{x}\right), \frac{1}{2}\left(d y_{i} \otimes d y_{j}+d y_{j} \otimes d y_{i}\right) .
$$


The corresponding wave operator is thus a totally characteristic, or b-, operator, $\square \in \operatorname{Diff}_{\mathrm{b}}^{2}(M)$, i.e. is the sum of products of up to two factors of elements of $\mathcal{V}_{\mathrm{b}}(M)$, with $\mathcal{C}^{\infty}(M)$ coefficients.

Invariantly, $\mathcal{V}_{\mathrm{b}}(M)$ is the space of all $\mathcal{C}^{\infty}$ sections of a vector bundle, ${ }^{\mathrm{b}} T M$, with local basis $x \partial_{x}, \partial_{y_{j}}$. The dual bundle of ${ }^{\mathrm{b}} T M$ is denoted by ${ }^{\mathrm{b}} T^{*} M$; it has a local basis of $\frac{d x}{x}, d y_{j}$. Thus, the tensors in (1.2) span (over $\mathcal{C}^{\infty}(M)$ ) the space of sections of the symmetric tensor power $S^{2 \mathrm{~b}} T^{*} M$ in a local coordinate chart. A bmetric then is a non-degenerate smooth section of $S^{2 \mathrm{~b}} T^{*} M$, i.e. a non-degenerate symmetric bilinear form on the fibers of ${ }^{\mathrm{b}} T M$ smoothly depending on the base point; a Lorentzian b-metric is one of signature $(1, n-1)$.

In the concrete example of the compactification of Kerr-de Sitter space described above, we simply have $x \partial_{x}=-\partial_{t_{*}}$ and $\frac{d x}{x}=-d t_{*}$, hence ${ }^{\mathrm{b}} T M$ and ${ }^{\mathrm{b}} T^{*} M$ can be thought of as 'uniform versions' of $T M^{\circ}$ and $T^{*} M^{\circ}$ up to the ideal boundary $x=0$. Further, a special class of elements of $\mathcal{C}^{\infty}(M)$ is given by the $t_{*}$-independent smooth functions on $X$, and in general, elements of $\mathcal{C}^{\infty}(M)$, by virtue of their smooth dependence on $x=e^{-t_{*}}$, differ from a $t_{*}$-independent function by a function bounded by $C e^{-t_{*}}$. (Since the choice $x=e^{-t_{*}}$ of boundary defining function of future infinity is arbitrary - any $x=e^{-\gamma t_{*}}, \gamma \in \mathcal{C}^{\infty}(X)$, would work as well the space $\mathcal{C}^{\infty}(M)$ is not quite natural, hence we will later amend it by adding to it the space $H_{\mathrm{b}}^{\infty}$ of conormal functions, see below, in order to obtain a space which $i s$ independent of the choice of b-compactification.) Thus, in this example, smooth b-metrics are asymptotically stationary, i.e. differ from a stationary metric by a symmetric 2-tensor which is exponentially decaying (measuring its size using a stationary Riemannian metric, or in fact any smooth Riemannian b-metric) in $t_{*}$.

In order to compress notation for elements of $\mathcal{V}_{\mathrm{b}}(M)$ applied to a function $u$, it is convenient to introduce the notation

$$
{ }^{\mathrm{b}} d u=\left(x \partial_{x} u\right) \frac{d x}{x}+\sum_{j}\left(\partial_{y_{j}} u\right) d y_{j}
$$

in terms of local coordinates. This is simply the differential $d u$ of $u$, rewritten in terms of the 1 -forms $\frac{d x}{x}$ and $d y_{j}$ dual to the vector fields $x \partial_{x}$ and $\partial_{y_{j}}$, thus it is in fact invariantly defined. Note that when one writes e.g. $a\left(u,{ }^{\mathrm{b}} d u\right)$, one could instead, at least locally, write

$$
a\left(u, x \partial_{x} u, \partial_{y_{1}} u, \ldots, \partial_{y_{n-1}} u\right)
$$

the ${ }^{\mathrm{b}} d u$ notation is more concise and invariant. We note that ${ }^{\mathrm{b}} d$ preserves reality.

We now discuss Sobolev spaces from the point of view of b-analysis: We measure regularity with respect to $\mathcal{V}_{\mathrm{b}}(M)$, and for non-negative integer $s$, one lets $H_{\mathrm{b}}^{s}(M)$ be the set of (complex-valued) $u \in L_{\mathrm{b}}^{2}(M)$ such that $V_{1} \ldots V_{j} u \in L_{\mathrm{b}}^{2}(M)$ for $j \leq s$ and $V_{1}, \ldots, V_{j} \in \mathcal{V}_{\mathrm{b}}(M)$. Here, $L_{\mathrm{b}}^{2}$ is the $L^{2}$-space with respect to any b-metric (such as the Kerr-de Sitter metric) which in local coordinates is thus given by a b-density, which is a positive smooth multiple of $x^{-1}\left|d x d y_{1} \ldots d y_{n-1}\right|$. Further, one introduces the weighted Sobolev spaces $H_{\mathrm{b}}^{s, \alpha}(M)=x^{\alpha} H_{\mathrm{b}}^{s}(M)$; we will often also work with the spaces $H_{\mathrm{b}}^{s, \alpha}(M ; \mathbb{R})$ of real-valued elements without making this explicit in the notation. $H_{\mathrm{b}}^{s, \alpha}(M)$-sections of vector bundles are defined by local trivializations. In our Kerr-de Sitter setup, the Sobolev spaces $H_{\mathrm{b}}^{s, \alpha}$ on $\Omega$ are defined by restriction, with the norm of $u \in H_{\mathrm{b}}^{s, \alpha}$ given by

$$
\|u\|_{H_{\mathrm{b}}^{s, \alpha}}^{2}=\sum_{j \leq s, 1 \leq i_{1} \leq \ldots \leq i_{j} \leq N}\left\|V_{i_{1}} \ldots V_{i_{j}} u\right\|_{L_{\mathrm{b}}^{2}(\Omega)}^{2},
$$


where $\left\{V_{1}, \ldots, V_{N}\right\}$ spans $\mathcal{V}_{\mathrm{b}}(M)$ over $\mathcal{C}^{\infty}(M)$. We point out that the b-differential ${ }^{\mathrm{b}} d$, defined locally by

$$
{ }^{\mathrm{b}} d u=\left(x \partial_{x} u\right) \frac{d x}{x}+\sum_{j}\left(\partial_{y_{j}} u\right) d y_{j}
$$

maps $H_{\mathrm{b}}^{s, \alpha}(M)$ to $H_{\mathrm{b}}^{s-1, \alpha}\left(M ;{ }^{\mathrm{b}} T^{*} M\right)$.

By the compactness of $\Omega$, norms on $H_{\mathrm{b}}^{s, \alpha}$ corresponding to different choices of spanning set and b-density are equivalent. We stress that this statement is a purely topological one, i.e. does not rely on any metric; only the choice of compactification $M$ (and thus $\Omega$ ) was motivated by the stationary nature of the Kerr-de Sitter metric, but even then, different choices of boundary defining function $e^{-\gamma t_{*}}, \gamma \in \mathcal{C}^{\infty}(X)$, as above, while changing the smooth structure of the compactification, yield the same b-Sobolev spaces. Likewise, norms of $H_{\mathrm{b}}^{s, \alpha}$-sections of a vector bundle $E$ over $\Omega$ can be defined using any positive definite metric on $E$ - again, by compactness of $\Omega$, all such choices give equivalent norms.

From the stationary point of view, the Sobolev space $H_{\mathrm{b}}^{s, \alpha}$ on $\Omega$ is the weighted Sobolev space $e^{-\alpha t_{*}} H^{s}$, with $H^{s}$ defined in (1.1); for sections of vector bundles $E$ over $\Omega$, one first identifies $E$ with the pullback of $\left.E\right|_{X}$ ( $X$ a spatial slice as before, intersected with $\Omega$ ), equipping $\left.E\right|_{X}$ with any positive definite fiber metric, by the map $\mathbb{R} \times X \ni\left(t_{*}, y\right) \mapsto y$, in order to obtain a $t_{*}$-independent bundle and metric on it, and one can then define norms of $E$-valued $H_{\mathrm{b}}^{s, \alpha}$-sections using local trivializations of $\left.E\right|_{X}$. In summary, adopting the 'b' point of view in the present context is a natural and geometric replacement for the use of the stationary nature of the spacetime, and a very useful one for the analysis, as we will see in the sequel.

Rephrasing and generalizing the setup of Theorem 1, the wave equations we study include those of the form

$$
\square_{g(u, \mathrm{~b} d u)} u=f+q\left(u,{ }^{\mathrm{b}} d u\right),
$$

where $g(0,0)=g_{0}$, and for each $p \in M, g_{p}\left(v_{0}, v\right): \mathbb{R} \oplus{ }^{\mathrm{b}} T_{p}^{*} M \rightarrow \mathrm{S}^{2} \mathrm{~b} T_{p}^{*} M$, depending smoothly on $p$, and

$$
q\left(u,{ }^{\mathrm{b}} d u\right)=\sum_{j=1}^{N^{\prime}} a_{j} u^{e_{j}} \prod_{k=1}^{N_{j}} X_{j k} u, \quad e_{j}, N_{j} \in \mathbb{N}_{0}, N_{j}+e_{j} \geq 2,
$$

with

$$
a_{j} \in \mathcal{C}^{\infty}(M), X_{j k} \in \mathcal{V}_{\mathrm{b}}(M)
$$

real; in fact, since $\mathcal{C}^{\infty}(M)$ is not a natural space as discussed above, we relax (1.3) to

$$
a_{j} \in \mathcal{C}^{\infty}(M)+H_{\mathrm{b}}^{\infty}(M), X_{j k} \in\left(\mathcal{C}^{\infty}+H_{\mathrm{b}}^{\infty}\right) \mathcal{V}_{\mathrm{b}}(M) ;
$$

we note that the space $\mathcal{C}^{\infty}(M)+H_{\mathrm{b}}^{\infty}(M)$ is independent of the choice of b-compactification. Generalizing the forcing as well, the more natural version of Theorem 1 is, with further generalization given in Theorems 3 and 4 :

Theorem 2. Let $s \in \mathbb{R}$. For $g(0,0)=g_{0}$ a Kerr-de Sitter metric with angular momentum $|a| \ll M_{\bullet}$ as above, and for $\alpha>0$ sufficiently small and $f \in H_{\mathrm{b}}^{\infty, \alpha}$ real-valued with sufficiently small $H_{\mathrm{b}}^{12, \alpha}$-norm (depending on $s$ ), the wave equation $\square_{g\left(u,{ }^{\mathrm{b}} d u\right)} u=f+q\left(u,{ }^{\mathrm{b}} d u\right)$ in $\Omega^{\circ}$, with vanishing Cauchy data, and with $q$ as above with $N_{j} \geq 1$ for all $j$, has a unique real smooth (in $\Omega^{\circ}$ ) global forward solution of 
the form $u=u_{0}+\widetilde{u}, \widetilde{u} \in H_{\mathrm{b}}^{s, \alpha}, u_{0}=c \chi, c \in \mathbb{R}$, with $\chi \in \mathcal{C}^{\infty}(M)$ identically 1 near $\partial M$.

Further, the analogous conclusion, in fact for $s=\infty$, holds for the Klein-Gordon equation $\square-m^{2}$ with $m>0$ sufficiently small, without the presence of the $u_{0}$ term, i.e. for $\alpha>0, m>0$ sufficiently small, if $f \in H_{\mathrm{b}}^{\infty, \alpha}(\Omega)$ has sufficiently small $H_{\mathrm{b}}^{12, \alpha}$-norm, $\left(\square_{g\left(u,{ }^{\mathrm{b}} d u\right)}-m^{2}\right) u=f+q\left(u,{ }^{\mathrm{b}} d u\right)$ in $\Omega^{\circ}$ has a unique, smooth in $\Omega^{\circ}$, global forward solution $u \in H_{\mathrm{b}}^{\infty, \alpha}$.

One can work over the complex numbers as well, the only change being that one needs to assume $g_{p}\left(v_{0}, v\right): \mathbb{C} \oplus{ }^{\mathbb{C b}} T_{p}^{*} M \rightarrow \mathrm{S}^{2} \mathrm{~b} T_{p}^{*} M$ now, i.e. taking complexvalued arguments, but still producing a real symmetric 2 -tensor. We will in fact consider the complex-valued setting in the rest of the paper, since the real-valued case follows as a special case.

The natural notion of asymptotically Kerr-de Sitter spaces in the context of the present paper is suggested by this result, namely, one would reasonably thus call $\left(M, g\left(u,{ }^{\mathrm{b}} d u\right)\right)$ for a function $u$ as in the above Theorem; hence, for us, an asymptotically Kerr-de Sitter space is a metric perturbation of a Kerr-de Sitter space within the class of sections of $S^{2 \mathrm{~b}} T^{*} M$ with regularity $\mathcal{C}^{\infty}+H_{\mathrm{b}}^{s, \alpha}, \alpha>0$, for $s$ large or $s=\infty$.

For the proof of Theorem 2, we refer to Corollaries 5.13 and 5.17, which are special cases of Theorems 5.10 and 5.16. For any fixed amount of regularity of the solution, our arguments only require a finite number of derivatives: Indeed, for sufficiently large $s_{0}, C \in \mathbb{R}$ and for $s \geq s_{0}$, it is sufficient to assume $f \in H_{\mathrm{b}}^{C s, \alpha}$, with small $H_{\mathrm{b}}^{12, \alpha}$-norm, to ensure the existence of a unique global forward solution $u$ with $H_{\mathrm{b}}^{s, \alpha}$-regularity, i.e. with $\widetilde{u} \in H_{\mathrm{b}}^{s, \alpha}$ in the case of wave equations, $u \in H_{\mathrm{b}}^{s, \alpha}$ in the case of Klein-Gordon equations; see Remark 5.12 for details.

We now discuss previous results on Kerr-de Sitter space and its perturbations. There seems to be little work on non-linear equations in Kerr-de Sitter type settings; indeed the only paper the authors are aware of is the earlier paper [33] of the authors in which the semilinear Klein-Gordon equation was studied (with small data wellposedness shown) with non-linearity depending on $u$ only, so that the losses due to the trapping could still be handled by a contraction mapping argument. In addition, the same paper also analyzed non-linearities depending on ${ }^{\mathrm{b}} d u$ provided these had a special structure at the trapped set. There is more work on the linear equation on perturbations of de Sitter-Schwarzschild and Kerr-de Sitter spaces: a rather complete analysis of the asymptotic behavior of solutions of the linear wave equation was given in [51], upon which the linear analysis of the present paper is ultimately based. Previously in exact Kerr-de Sitter space and for small angular momentum, Dyatlov $[21,20]$ has shown exponential decay to constants, even across the event horizon; see also the more recent work of Dyatlov [19]. Further, in de SitterSchwarzschild space (non-rotating black holes) Bachelot [3] set up the functional analytic scattering theory in the early 1990s, while later Sá Barreto and Zworski [45] and Bony and Häfner [6] studied resonances and decay away from the event horizon, Dafermos and Rodnianski in [14] showed polynomial decay to constants in this setting, and Melrose, Sá Barreto and Vasy [42] improved this result to exponential decay to constants. There is also physics literature on the subject, starting with Carter's discovery of this space-time [8, 7], either using explicit solutions in special cases, or numerical calculations, see in particular [54], and references therein. We 
also refer to the paper of Dyatlov and Zworski [24] connecting recent mathematical advances with the physics literature.

While it received more attention, the linear, and thus the non-linear, equation on Kerr space (which has vanishing cosmological constant) does not fit directly into our setting; see the introduction of [51] for an explanation and for further references and [15] for more background and additional references. Some of the key works in this area include the polynomial decay on Kerr space which was shown recently by Tataru and Tohaneanu [48, 47] and Dafermos, Rodnianski and ShlapentokhRothman [10, 11, 16], after pioneering work of Kay and Wald in [37] and [52] in the Schwarzschild setting. Andersson and Blue [1] proved a decay result for the Maxwell system on slowly rotating Kerr spaces; see also the earlier work of Bachelot [2] in the Schwarzschild setting. The crucial normal hyperbolicity of the trapping, corresponding to null-geodesics that do not escape through the event horizons, in Kerr space was realized and proved by Wunsch and Zworski [53]; later Dyatlov extended and refined the result $[22,23]$. Note that a stronger version of normal hyperbolicity, called $r$-normal hyperbolicity and proved for Kerr in [53] as well, is a notion that is stable under perturbations.

On the non-linear side, Luk [38] established global existence for forward problems for semilinear wave equations on Kerr space under a null condition, and Dafermos, Holzegel and Rodnianski [9] constructed backward solutions for Einstein's equations on Kerr space. (There was also recent work by Marzuola, Metcalfe, Tataru and Tohaneanu [39] and Tohaneanu [50] on Strichartz estimates, which are applied to the study of semilinear wave equations with power non-linearities, and by Donninger, Schlag and Soffer [18] on $L^{\infty}$ estimates on Schwarzschild black holes, following $L^{\infty}$ estimates of Dafermos and Rodnianski [13, 12], of Blue and Soffer [5] on nonrotating charged black holes giving $L^{6}$ estimates, and of Finster, Kamran, Smoller and Yau $[25,26]$ on Dirac waves on Kerr.)

In the next section, $\S 2$, we explain the ingredients of the proof of Theorem 2, and we also state natural generalizations. At the end of that section we provide a detailed roadmap through this paper.

The authors are very grateful to Semyon Dyatlov for providing a preliminary version of his manuscript [23] and for discussions about it, as well as for pointing out the reference [35]. They are also very grateful to Maciej Zworski and three anonymous referees for comments that greatly improved the exposition. They are also thankful to Gunther Uhlmann, Richard Melrose and Rafe Mazzeo for comments and their interest in this project.

\section{Overview OF THE PROOF AND THE MORE GENERAL RESUlTS}

Having stated the result, we now explain why it holds. It is best to begin with the underlying linear equation; after all, the non-linearity is 'just' a rather serious perturbation! In general, the analysis of b-differential operators (locally finite sums of finite products of elements of $\left.\mathcal{V}_{\mathrm{b}}(M)\right)$, such as $\square_{g} \in \operatorname{Diff}_{\mathrm{b}}^{2}(M)$, has two ingredients, corresponding to the two orders, smoothness and decay, of the Sobolev spaces:

(1) b-regularity analysis. This provides the framework for understanding PDE at high b-frequencies, which in non-degenerate situations involves the bprincipal symbol and perhaps a subprincipal term. This is sufficient in order to control solutions $u$ in $H_{\mathrm{b}}^{s, r}$ in terms of their $H_{\mathrm{b}}^{s^{\prime}, r}$-norm, $s^{\prime}<s$, i.e. 
in terms of the norm of $u$ in a space with lower regularity, but no additional decay. Since for the inclusion $H_{\mathrm{b}}^{s, r} \rightarrow H_{\mathrm{b}}^{s^{\prime}, r^{\prime}}$ to be compact one needs both $s>s^{\prime}$ and $r>r^{\prime}$, this does not control the problem modulo relatively compact errors.

(2) Normal operator analysis. This provides a framework for understanding the decay properties of solutions of the PDE. The normal operator is obtained by freezing coefficients of the differential operator $L$ at $\partial M$ to obtain a dilation-invariant b-operator $N(L)$. One then Mellin transforms the normal operator in the normal variable to obtain a family of operators $\widehat{L}(\sigma)$, depending on the Mellin-dual variable $\sigma$. The b-regularity analysis, in non-degenerate situations, gives control of this family $\widehat{L}(\sigma)$ in a Fredholm sense, uniformly as $|\sigma| \rightarrow \infty$ with $\operatorname{Im} \sigma$ bounded. However, in any such strip, $\widehat{L}(\sigma)^{-1}$ will still typically have finitely many poles $\sigma_{j}$; these poles, called resonances, dictate the asymptotic behavior of solutions of the PDE.

In order to have a Fredholm operator $L$, one needs to work in spaces such as $H_{\mathrm{b}}^{s, r}$, where $r$ is such that there are no resonances $\sigma_{j}$ with $\operatorname{Im} \sigma_{j}=-r$. One can also work in slightly more general spaces, such as $\mathcal{X}^{s, r}:=\mathbb{C} \oplus H_{\mathrm{b}}^{s, r}, r>0$, identified with a space of distributions via $u=u_{0}+\widetilde{u}, \widetilde{u} \in H_{\mathrm{b}}^{\infty, \alpha}, u_{0}=c \chi$, corresponding to $(c, \widetilde{u}) \in \mathbb{C} \oplus H_{\mathrm{b}}^{s, r}$.

The global analysis of linear (and semilinear) wave-type equations on asymptotically Kerr-de Sitter spacetimes in this framework, which we will recall momentarily, was carried out in $[51,33]$, and the analysis in the present paper follows these earlier works closely.

Now, the b-regularity analysis for our non-elliptic equation involves the (null)bicharacteristic flow. In view of the version of Hörmander's theorem on propagation of singularities in this setting, and in view of the a priori control on Cauchy data at $H_{1}$, what one would like is that all bicharacteristics tend to $T_{H_{1}}^{*} M$ in one direction. Moreover, for the purposes of the adjoint problem, which effectively imposes Cauchy data at $H_{2}$, one would like that the bicharacteristics tend to ${ }^{\mathrm{b}} T_{\mathrm{H}_{2}}^{*} \mathrm{M}$ in the other direction.

Unfortunately, bicharacteristics within ${ }^{\mathrm{b}} T_{X}^{*} M$ can never leave this space, and thus will not tend to $T_{H_{1}}^{*} M$. This is mostly resolved, however, by the conormal bundle of the horizons at $X$, which give rise to a bundle of saddle points for the bicharacteristic flow. Since the flow is homogeneous, it is convenient to consider it in ${ }^{\mathrm{b}} S^{*} M=\left({ }^{\mathrm{b}} T^{*} M \backslash o\right) / \mathbb{R}^{+}$. The characteristic set $\Sigma \subset{ }^{\mathrm{b}} S^{*} M$ has two components $\Sigma_{ \pm}$, with $\Sigma_{-}$forward-oriented (i.e. future oriented time functions increase along null-bicharacteristics in $\left.\Sigma_{-}\right), \Sigma_{+}$backward oriented. Then the images of the conormal bundles of the horizons in the cosphere bundle are submanifolds $L_{ \pm} \subset \Sigma_{ \pm}$of ${ }^{\mathrm{b}} S_{X}^{*} M$, with one-dimensional stable $(-) /$ unstable $(+)$ manifold $\mathcal{L}_{ \pm}$transversal to ${ }^{\mathrm{b}} S_{X}^{*} M$. (The flow within $L_{ \pm}$need not be trivial; if it is, one has radial points, as in the $a=0$ de Sitter-Schwarzschild space. However, for simplicity we refer to the $L_{ \pm}$ estimates as radial point estimates in general.) The realistic ideal situation, called a non-trapping one, then is if all (null-)bicharacteristics in ${ }^{\mathrm{b}} S_{\Omega}^{*} M \cap\left(\Sigma_{+} \backslash L_{+}\right)$tend to b $S_{H_{2}}^{*} M \cup L_{+}$in the backward direction, and ${ }^{\mathrm{b}} S_{H_{1}}^{*} M \cup L_{+}$in the forward direction, with a similar statement for $\Sigma_{-}$, with backward and forward interchanged. Notice that due to the assumption on the one-dimensional stable/unstable manifold being transversal to ${ }^{\mathrm{b}} S_{X}^{*} M$, there cannot be non-trivial bicharacteristics in ${ }^{\mathrm{b}} S^{*} M$ tending to $L_{+}$in both the forward and the backward direction, since a bicharacteristic is 
either completely in ${ }^{\mathrm{b}} S_{X}^{*} M$, or completely outside it. In this non-trapping setting the only subtlety is that the propagation estimates through $L_{ \pm}$require that the differentiability order $s$ and the decay order $r$ be related by $s>\frac{1}{2}+\beta r$ for a suitable $\beta>0$ (dictated by the Hamilton dynamics at $L_{ \pm}$), i.e. the more decay one wants, the higher the regularity needs to be.

This is still not the case in Kerr-de Sitter space, though it is true for neighborhoods of the static patch in de Sitter space, and its perturbations. The additional ingredient for Kerr-de Sitter space is normally hyperbolic trapping, introduced in this context by Wunsch and Zworski [53], given by smooth submanifolds $\Gamma^{ \pm} \subset \Sigma_{ \pm}$. Here $\Gamma^{ \pm}$are invariant submanifolds for the Hamilton flow, given by the transversal intersection of locally defined smooth, Hamilton flow invariant, $\Gamma^{ \pm}=\Gamma_{+}^{ \pm} \cap \Gamma_{-}^{ \pm}$, with $\Gamma_{-}^{ \pm} \subset \Sigma$ transversal to ${ }^{\mathrm{b}} S_{X}^{*} M \cap \Sigma$, and $\Gamma_{+}^{ \pm} \subset{ }^{\mathrm{b}} S_{X}^{*} M \cap \Sigma$. Combining results of $[22,23]$ (which would work directly in a dilation invariant setting) and [32] we show that for $r>0$ sufficiently small, one can propagate $H_{\mathrm{b}}^{s, r}$ estimates through $\Gamma^{ \pm}$. This suffices to complete the b-regularity setup if the non-trapping requirement is replaced by: All (null-)bicharacteristics in ${ }^{\mathrm{b}} S_{\Omega}^{*} M \cap\left(\Sigma_{+} \backslash\left(L_{+} \cup \Gamma^{+}\right)\right)$tend to either ${ }^{\mathrm{b}} S_{H_{2}}^{*} M \cup L_{+} \cup \Gamma^{+}$in the backward direction, and ${ }^{\mathrm{b}} S_{H_{1}}^{*} M \cup L_{+} \cup \Gamma^{+}$in the forward direction, with the tending to $\Gamma^{+}$allowed in only one of the forward and backward directions, with a similar statement for $\Sigma_{-}$, with backward and forward interchanged. Finally, this is satisfied in Kerr-de Sitter space, and also in its b-perturbations (the whole setup is perturbation stable).

Next, one needs to know about the resonances of the operator. For the wave operator, the only resonance with non-negative imaginary part is 0 , with the kernel of $\widehat{L}(\sigma)$ one dimensional, consisting of constants. Since strips can only have finitely many resonances, there is $r>0$ such that in $\operatorname{Im} \sigma \geq-r$ the only resonance is 0 ; then $H_{\mathrm{b}}^{s, r} \oplus \mathbb{C}$ works for our Fredholm setup. For the Klein-Gordon equation with $m>0$ small, the $m=0$ resonance at 0 moves to $\sigma_{1}=\sigma_{1}(m)$ inside $\operatorname{Im} \sigma<0$, see $[21,33]$. Thus, one can either work with $H_{\mathrm{b}}^{s, r^{\prime}}$ where $r^{\prime}$ is sufficiently small (depending on $m$ ), or with $H_{\mathrm{b}}^{s, r} \oplus \mathbb{C}$, though with $\mathbb{C}$ now identified with $c x^{i \sigma_{1}} \chi$.

We now discuss the non-linear terms. Here the basic point is that $H_{\mathrm{b}}^{s, 0}$ is an algebra if $s>n / 2$, and thus for such $s$, products of elements of $H_{\mathrm{b}}^{s, r}$ possess even more decay if $r>0$, but they become more growing if $r<0$. Thus, one is forced to work with $r \geq 0$.

First, with the simplest semilinear equation, with no derivatives in the nonlinearity $q$ (so $N_{j} \geq 2$ is replaced by $N_{j}=0$ ), the regularity losses due to the normally hyperbolic trapping are in principle sufficiently small to allow for a contraction mapping principle (Picard iteration) based argument. However, for the actual wave equation on Kerr-de Sitter space, the 0-resonance prohibits this, as the iteration maps outside the space $H_{\mathrm{b}}^{s, r} \oplus \mathbb{C}$. Thus, it is the semilinear KleinGordon equation that is well-behaved from this perspective, and this was solved by the authors in [33]. On the other hand, if derivatives are allowed, with an at least quadratic behavior in ${ }^{\mathrm{b}} d u$, then the non-linearity annihilates the 0-resonance. Unfortunately, since the normally hyperbolic estimate loses $1+\epsilon$ derivatives, as opposed to the usual real principal type/radial point loss of one derivative, the solution operator for $\square_{g}$ will not map $q\left(u,{ }^{\mathrm{b}} d u\right)$ back into the desired Sobolev space, preventing a non-linear analysis based on the contraction mapping principle.

Fortunately, the Nash-Moser iteration is designed to deal with just such a situation. In this paper we adapt the iteration to our requirements, and in particular 
show that semilinear equations of the kind just described are in fact solvable. In particular, we prove that all the estimates used in the linear problems are tame. Here we remark that Klainerman's early work on global solvability involved the Nash-Moser scheme [35], though this was later removed by Klainerman and Ponce [36]. In the present situation the loss of derivatives seems much more serious, however, due to the trapping, so it seems unlikely that the solution scheme can be made more 'classical'.

However, we are also interested in quasilinear equations. Quasilinear versions of the above non-trapping scenario were studied by the first author [30], who showed the solvability of quasilinear wave equations on perturbations of de Sitter space. The key ingredient in dealing with quasilinear equations is to allow operators with coefficients with regularity the same kind as what one is proving for the solutions, in this case $H_{\mathrm{b}}^{s, r}$-regularity; the technical tools for dealing with such operators were developed in [30], following Beals and Reed [4]. All of the smooth linear ingredients (microlocal elliptic regularity, propagation of singularities, radial points) have their analogue for $H_{\mathrm{b}}^{s, r}$ coefficients if $s$ is sufficiently large. Thus, in [30] a Picardtype iteration, $u_{k+1}=\square_{g\left(u_{k}\right)}^{-1}\left(f+q\left(u_{k}, \mathrm{~b} d u_{k}\right)\right)$ was used to solve the quasilinear wave equations on de Sitter space. Notice that $\square_{g\left(u_{k}\right)}$ has non-smooth coefficients; indeed, these lie in a weighted b-Sobolev space.

In our Kerr-de Sitter situation there is normally hyperbolic trapping. However, notice that as we work in decaying Sobolev spaces modulo constants, $\square_{g(u)}$ differs from a Kerr-de Sitter operator with smooth coefficients, $\square_{g(c)}$, by one with exponentially (in $t_{*}$ ) decaying coefficients. This means that one can combine the smooth coefficient normally hyperbolic theory, as in the work of Dyatlov [22], with a tame estimate in $H_{\mathrm{b}}^{s, r}$ with $r<0$; the sign of $r$ here is a crucial gain since for $r<0$ the propagation estimates through normally hyperbolic trapped sets behave in exactly the same way as real principal type estimates. In combination this provides the required tame estimates for Kerr-de Sitter wave equations: Using the notation of Theorem 2 and its setup (thus, allowing $g$ to depend on derivatives of $u$ as well), and with $u=c \chi+\widetilde{u}, c \in \mathbb{R}, \widetilde{u} \in H_{\mathrm{b}}^{s+4, \alpha}$, the tame estimate takes the form

$$
\|w\|_{\mathcal{X}^{s, \alpha}} \leq C\left(s,\|u\|_{\mathcal{X}^{s_{0}, \alpha}}\right)\left(\|f\|_{H_{\mathrm{b}}^{s+3, \alpha}}+\|f\|_{H_{\mathrm{b}}^{s_{0}, \alpha}}\|u\|_{\mathcal{X}^{s+4, \alpha}}\right)
$$

for $w=b \chi+\widetilde{w}, b \in \mathbb{R}, \widetilde{w} \in H_{\mathrm{b}}^{s, \alpha}$, solving $\square_{g\left(u,{ }^{\mathrm{b}} d u\right)} w=f$, where we use the norm $\|w\|_{\mathcal{X}^{s, \alpha}}:=|b|+\|\widetilde{w}\|_{H_{\mathrm{b}}^{s, \alpha}}$, likewise for $u=a \chi+\widetilde{u}$, and $s_{0}$ is a suitable fixed number, while $s$ can be chosen arbitrarily large. The point of a tame estimate like (2.1) for $w$ is that one loses a fixed number of derivatives, independent of $s$, relative to the forcing $f$, and moreover the estimate is linear in the high regularity norms of the forcing $f$ and the coefficients, coming from $u$, of the operator $\square_{g(u, b d u)}$. The idea of the proof of the estimate (2.1) is to rewrite $\left.\square_{g(u, b} d u\right) w=f$ as $\square_{g(c, 0)} w=$ $f-\left(\square_{g(u, \mathrm{~b} d u)}-\square_{g(c, 0)}\right) w$ and treating the second term on the right as an error term in the sense that it has better a priori decay than $w$, even though it has less regularity; then the normal operator analysis allows one to deduce further partial asymptotics for $w$ from the rewritten equation. Tame regularity estimates for the equation $\square_{g(u, \mathrm{~b} d u)} w=f$ itself, using the dynamical structure of Kerr-de Sitter space as described above, then allow one to regain the loss of regularity to a large extent. The tame-ness comes about by a careful analysis of these microlocal estimates, the fundamental ingredient being a simple Moser-type estimate for products of Sobolev functions, which on $\mathbb{R}^{n}$ is of the form $\|u v\|_{H^{s}} \lesssim\|u\|_{H^{s}}\|v\|_{H^{s_{0}}}+\|u\|_{H^{s_{0}}}\|v\|_{H^{s}}$ 
for $s \geq s_{0}, s_{0}$ sufficiently large but fixed. - Given (2.1), one can then use a simple version of the Nash-Moser iteration scheme given by Saint-Raymond [46] to complete the proof of the main theorem.

We emphasize that our treatment of quasilinear wave-type equations is systematic and general; indeed, our discussion of quasilinear waves in $\S 5$ takes place in a general geometric setting (but we restrict to scalar equations for brevity): To wit, quasilinear equations which at $X=\partial M$ are modelled on a finite dimensional family $L=L\left(v_{0}\right), v_{0} \in \mathbb{C}^{d}$ small corresponding to the zero resonances (thus the family is 0-dimensional without 0-resonances!), of smooth b-differential operators on a vector bundle with scalar principal symbol which has the bicharacteristic dynamics described above (radial sets, normally hyperbolic trapping, etc.) fit into it, provided two conditions hold for the normal operator (i.e. the dilation invariant model associated to $L$ at $\partial M):^{2}$

(1) First, the resonances for the model $L\left(v_{0}\right)$ have negative imaginary part, or if they have 0 imaginary part, the non-linearity annihilates them.

(2) Second, the normally hyperbolic trapping estimates of Dyatlov [22] hold for $\widehat{L}(\sigma)($ as $|\operatorname{Re} \sigma| \rightarrow \infty)$ in $\operatorname{Im} \sigma>-r_{0}$ for some $r_{0}>0$. In the semiclassical rescaling, with $\sigma=h^{-1} z, h=|\sigma|^{-1}$, this is a statement about $\widehat{L}_{h, z}=$ $h^{m} \widehat{L}\left(h^{-1} z\right), \operatorname{Im} z>-r_{0} h$. By Dyatlov's recent result ${ }^{3}[23]$ this indeed is the case if $\widehat{L}_{h, z}$ satisfies that at $\Gamma$ its skew-adjoint part, $\frac{1}{2 i}\left(\widehat{L}_{h, z}-\widehat{L}_{h, z}^{*}\right) \in$ $h \operatorname{Diff}_{\hbar}^{1}(X)$, for $z \in \mathbb{R}$ has semiclassical principal symbol bounded above by $h\left(\nu_{\min }-\epsilon\right) / 2$ for some $\epsilon>0$, where $\nu_{\min }$ is the minimal expansion rate in the normal directions at $\Gamma$; see [23, Theorem 1] and the remark below it (which allows the non-trivial skew-adjoint part, denoted by $Q$ there, microlocally at $\Gamma$ ).

It is important to point out that in view of the decay of the solutions either to 0 if there are no real resonances, or to the space of resonant states corresponding to real resonances, the conditions must be checked for at most a finite dimensional family of elements of the 'smooth' algebra $\Psi_{\mathrm{b}}(M)$, and moreover there is no need to prove tame estimates, deal with rough coefficients, etc., for this point, and one is in a dilation invariant setting, i.e. can simply Mellin transform the problem. Hence, in principle, solving wave-type equations on more complicated bundles is reduced to analyzing these two aspects of the associated linear model operator at infinity.

Concretely, we have the following two theorems on Kerr-de Sitter spaces:

Theorem 3. Let $M$ be a Kerr-de Sitter space with angular momentum $|a|<\frac{\sqrt{3}}{2} M_{\bullet}$ that satisfies [51, (6.13)] (this condition on $\Lambda, M_{\bullet}$ and a ensures non-trapping classical dynamics for the null-bicharacteristic flow for the normal operator family). Let $E$ a vector bundle over $M$ with a positive definite metric $k$ on $E$, and let $L_{g\left(u,{ }^{b} d u\right)} \in \operatorname{Diff}_{\mathrm{b}}^{2}(M ; E)$ have principal symbol $G=g^{-1}\left(u,{ }^{\mathrm{b}} d u\right)$ (times the identity), and suppose that $L_{0}=L_{g(0,0)}$ satisfies that

\footnotetext{
${ }^{2}$ The operator needs to be a second order differential operator, with principal symbol a Lorentzian dual metric, only if Cauchy hypersurfaces are used, as we do in the applications presented in this paper; otherwise, e.g. in situations where one can instead use complex absorption, the order of the operator is irrelevant.

${ }^{3}$ This could presumably also be seen from the work of Nonnenmacher and Zworski [44] by checking that this extension goes through without significant changes in the proof.
} 
(1) the large parameter principal symbol of $\frac{1}{2 i|\sigma|}\left(L_{0}-L_{0}^{*}\right)$, with the adjoint taken relative to $k|d g|$, at the trapped set $\Gamma$ is $<\nu_{\min } / 2$ as an endomorphism of $E$,

(2) $\widehat{L}_{0}(\sigma)$ has no resonances in $\operatorname{Im} \sigma \geq 0$.

Then for $\alpha>0$ sufficiently small, there exists ${ }^{4} d>0$ such that the following holds: If $f \in H_{\mathrm{b}}^{\infty, \alpha}(\Omega)$ has a sufficiently small $H_{\mathrm{b}}^{2 d}$-norm, then the equation $L_{g\left(u,{ }^{\mathrm{b}} d u\right)} u=$ $f+q\left(u,{ }^{\mathrm{b}} d u\right)$ has a unique, smooth in $M^{\circ}$, global forward solution $u \in H_{\mathrm{b}}^{\infty, \alpha}(\Omega)$.

In particular, the conditions at $\Gamma$ for the theorem hold if $|a| \ll M_{\bullet}, E$ a tensor bundle over $M$ of finite rank (e.g. the bundle ${ }^{\mathrm{b}} \Lambda^{*} M$ of differential forms or $S^{k \mathrm{~b}} T^{*} M$ of symmetric $k$-tensors $), L_{g\left(u,{ }^{\mathrm{b}} d u\right)}=\square_{g\left(u,{ }^{\mathrm{b}} d u\right)}$ the tensor d'Alembertian, or indeed if $L_{g\left(u,{ }^{\mathrm{b}} d u\right)}-\square_{g\left(u,{ }^{\mathrm{b}} d u\right)}$ is a 0th order operator, since hyperbolicity is shown in [51] in the full stated range of $a$, while for $a=0$, one can explicitly compute $\frac{1}{2 i}\left(L_{0}-L_{0}^{*}\right)$ at $\Gamma$ and verify the stated bound, where one takes adjoints with respect to a suitable Riemannian fiber metric $k$; in fact, to obtain this bound for all permitted spacetime parameters $M_{\bullet}$ and $\Lambda$, one needs to take $k$ to be a pseudodifferential Riemannian inner product, which one can indeed allow as well in the statement of the Theorem. See [29] for definitions and proofs.

Theorem 4. Let $\left(M, g_{0}\right)$ be a Kerr-de Sitter space with angular momentum $|a|<$ $\frac{\sqrt{3}}{2} M_{\bullet}$ that satisfies [51, (6.13)]. Let $E$ be a vector bundle over $M$ with a positive definite metric $k$ on $E$, and let $L_{g\left(u,{ }^{b} d u\right)} \in \operatorname{Diff}_{\mathrm{b}}^{2}(M ; E)$ have principal symbol $G=g^{-1}\left(u,{ }^{\mathrm{b}} d u\right)$ (times the identity), where $g(0,0)=g_{0}$. Suppose further that $L_{0}=L_{g(0,0)}$ is such that $\widehat{L}_{0}(\sigma)$ has a simple resonance at 0 , with resonant states spanned by $u_{0,1}, \ldots, u_{0, d}$, and no other resonances in $\operatorname{Im} \sigma \geq 0$. Consider the family $\widehat{L}_{g\left(u_{0},{ }^{\mathrm{b}} d u_{0}\right)}(\sigma), u_{0} \in \operatorname{Span}\left\{u_{0,1}, \ldots, u_{0, d}\right\}$ with small enough norm. Suppose that

(1) this family only has resonances at 0 in $\operatorname{Im} \sigma \geq 0$, and these are given by $\operatorname{Span}\left\{u_{0,1}, \ldots, u_{0, d}\right\}$,

(2) the large parameter principal symbol of $\frac{1}{2 i|\sigma|}\left(L_{0}-L_{0}^{*}\right)$, with the adjoint taken relative to $k|d g|$, at the trapped set $\Gamma$ is $<\nu_{\min } / 2$,

(3) $q\left(u_{0},{ }^{\mathrm{b}} d u_{0}\right)=0$ for $u_{0} \in \operatorname{Span}\left\{u_{0,1}, \ldots, u_{0, d}\right\}$.

Let $s \in \mathbb{R}$. Then for $\alpha>0$ sufficiently small (depending on $s$ ), there exists ${ }^{5} d>0$ such that the following holds: If $f \in H_{\mathrm{b}}^{\infty, \alpha}$ has a sufficiently small $H_{\mathrm{b}}^{2 d, \alpha}$-norm, then the equation $L_{g\left(u,{ }^{\mathrm{b}} d u\right)} u=f+q\left(u,{ }^{\mathrm{b}} d u\right)$ has a unique, smooth in $M^{\circ}$, global forward solution of the form $u=u_{0}+\widetilde{u}, \widetilde{u} \in H_{\mathrm{b}}^{s, \alpha}, u_{0}=\chi \sum_{j=1}^{d} c_{j} u_{0, j}, \chi \in \mathcal{C}^{\infty}(M)$ identically 1 near $\partial M$.

If moreover $\Gamma$ is uniformly normally hyperbolic for $\widehat{L}_{g\left(u_{0},{ }^{b} d u_{0}\right)}(\sigma)$, one can take $s=\infty$, i.e. obtain a solution $u$ as above, with $\widetilde{u} \in H_{\mathrm{b}}^{\infty, \alpha}$ now.

Here, 'uniformly normally hyperbolic' means that one has a continuous family $\Gamma=\Gamma_{u_{0}}$ of smooth trapped sets, with a continuous family of stable/unstable manifolds, with uniform bounds (within this family) on the normal expansion rates for the flow, which ensures that the normally hyperbolic estimates are uniform within the family (for small $u_{0}$ ); see the discussion around (4.27) for details.

\footnotetext{
${ }^{4}$ See the proof of this theorem in $\S 5.4$, in particular (5.27), for the value of $d$.

${ }^{5}$ The value of $d$ is given in (5.27) in the course of the proof of this theorem in $\S 5.4$.
} 
We stress again that Theorems 3 and 4 hold under the general assumptions described above; we restrict to quasilinear equations on Kerr-de Sitter spaces merely for concreteness. See also Remark 5.18.

Again, the conditions at $\Gamma$ for the theorem hold if $|a| \ll M_{\bullet}, E$ a tensor bundle, if $L_{g(u, \mathrm{~b} d u)}-\square_{g(u, \mathrm{~b} d u)}$ is a 0th order operator, $\square_{g(u, \mathrm{~b} d u)}$ the tensor d'Alembertian, since the structurally stable $r$-normally hyperbolic statement is shown in [51] (which implies the uniform normal hyperbolicity required in the theorem), while for $a=0$, $\frac{1}{2 i}\left(L_{0}-L_{0}^{*}\right)$ can be computed explicitly at $\Gamma$, as mentioned above, and upper bounds on this are stable under perturbations.

The uniform normal hyperbolicity condition at $\Gamma$ holds if $|a|<\frac{\sqrt{3}}{2} M_{\bullet}, E$ a tensor bundle, $L_{g\left(u,{ }^{\mathrm{b}} d u\right)}=\square_{g(u, \mathrm{~b} d u)}$ the tensor d'Alembertian, with $g\left(u_{0},{ }^{\mathrm{b}} d u_{0}\right)$ being a Kerr-de Sitter metric for $u_{0} \in \operatorname{Span}\left\{u_{0,1}, \ldots, u_{0, d}\right\}$ with small norm, since the hyperbolicity of $\Gamma$ was shown in this generality in [51]. (However, the computation of $\frac{1}{2 i}\left(L_{0}-L_{0}^{*}\right)$ for this range of $a$ is more involved and will not be pursued here.) While the assumption on $g\left(u_{0},{ }^{\mathrm{b}} d u_{0}\right)$ here is somewhat unnatural from the point of view of applications, a closely related assumption is expected to hold in the context of Einstein's field equations; this is subject of ongoing research.

The plan of the rest of this paper is the following. In $\S 3$ we show that the non-smooth pseudodifferential operators of [30] facilitate tame estimates (operator bounds, composition, etc.), with $\S 4$ establishing tame elliptic estimates in $\S 4.1$, tame real principal type and radial point estimates in $\S 4.2$ and tame estimates at normally hyperbolic trapping in $\S 4.3$ for $r<0$. In $\S 4.4$, we adapt Dyatlov's analysis at normally hyperbolic trapping given in [23] to our needs. Finally, in $\S 5$ we solve our quasilinear equations by first showing that the microlocal results of $\S 4$ combine with the high energy estimates for the relevant normal operators following from the discussion of $\S 4.4$ to give tame estimates for the forward propagator in $\S 5.1$, and then showing in $\$ 5.2$ that the Nash-Moser iteration indeed allows for solving our wave equations. $\$ 5.3$ then explains the changes required for quasilinear KleinGordon equations. Finally, in $\S 5.4$ we show how our methods apply in the general settings of Theorems 3 and 4 .

\section{TAME ESTIMATES IN THE NON-SMOOTH OPERATOR CALCULUS}

In this section we prove the basic tame estimates for the $H_{\mathrm{b}}$-coefficient, or simply non-smooth, b-pseudodifferential operators defined in [30].

We work on the half space $\overline{\mathbb{R}_{+}^{n}}$ with coordinates $z=(x, y) \in[0, \infty) \times \mathbb{R}^{n-1}$; the coordinates in the fiber of the b-cotangent bundle are denoted $\zeta=(\lambda, \eta)$, i.e. we write b-covectors as $\lambda \frac{d x}{x}+\eta d y$. We point out that under the change of coordinates $(0, \infty)_{x} \times \mathbb{R}_{y}^{n-1} \mapsto \mathbb{R}^{n},(x, y) \mapsto(-\log x, y)$, the non-smooth b-ps.d.o.s considered here become quantizations of symbols with standard (on $\mathbb{R}^{n}$ ) Sobolev regularity in the coefficients. However, once non-smooth and smooth b-ps.d.o.s interact (as they will in the study of microlocal regularity results at the boundary $x=0$ ), the b-picture, locally working on $\overline{\mathbb{R}_{+}^{n}}$, will be conceptually much cleaner than the Euclidean picture, locally working on $\mathbb{R}^{n}$ with a certain uniformity in one direction; hence we emphasize the ' $b$ ' point of view from the beginning.

3.1. Mapping properties. We start with the tame mapping estimate, Proposition 3.1, which essentially states that for non-smooth pseudodifferential operators $A$, a high regularity norm of $A u$ can be estimated by a high regularity norm of 
$A$ times a low regularity norm of $u$, plus a low regularity norm of $A$ times a high regularity norm of $u$. This is stronger than the a priori continuity estimate one gets from the bilinear map $(A, u) \mapsto A u$, which would require a product of high norms of both. In case $A$ is a multiplication operator, this is essentially a b-version of a (weak) Moser estimate, see Corollary 3.2.

Recall from [30] the symbol class

$$
S^{m ; 0} H_{\mathrm{b}}^{s}:=\left\{a(z, \zeta):\left\|\langle\zeta\rangle^{-m} a(z, \zeta)\right\|_{H_{\mathrm{b}}^{s}} \in L_{\zeta}^{\infty}\right\}
$$

with the norm

$$
\|a\|_{S^{m ; 0} H_{\mathrm{b}}^{s}}=\left\|\frac{\langle\xi\rangle^{s} \widehat{a}(\xi, \zeta)}{\langle\zeta\rangle^{m}}\right\|_{L_{\zeta}^{\infty} L_{\xi}^{2}}
$$

where $\widehat{a}$ denotes the Mellin transform in $x$ (which is the standard Fourier transform in $-\log x)$ and Fourier transform in $y$ of $a$. Left quantizations of such symbols, denoted $\operatorname{Op}(a) \in \Psi^{m ; 0} H_{\mathrm{b}}^{s}$, act on $u \in \dot{\mathcal{C}}_{\mathrm{c}}^{\infty}\left(\overline{\mathbb{R}_{+}^{n}}\right)$ by

$$
\mathrm{Op}(a) u(x, y)=(2 \pi)^{-n} \int x^{i \lambda} e^{i y \eta} a(x, y, \lambda, \eta) \widehat{u}(\lambda, \eta) d \lambda d \eta \text {. }
$$

Also recall

$$
S^{m ; k} H_{\mathrm{b}}^{s}=\left\{a \in S^{m ; 0} H_{\mathrm{b}}^{s}: \partial_{\zeta}^{\alpha} a \in S^{m-|\alpha| ; 0} H_{\mathrm{b}}^{s},|\alpha| \leq k\right\} .
$$

and $\Psi^{m ; k} H_{\mathrm{b}}^{s}=\mathrm{Op} S^{m ; k} H_{\mathrm{b}}^{s}$. For brevity, we will use the following notation for Sobolev, symbol class and operator class norms, with the distinction between symbolic and b-Sobolev norms being clear from the context:

$$
\begin{gathered}
\|u\|_{s}:=\|u\|_{H_{\mathrm{b}}^{s}}, \quad\|u\|_{s, r}:=\|u\|_{H_{\mathrm{b}}^{s, r}} \\
\|a\|_{m, s}:=\|a\|_{S^{m ; 0} H_{\mathrm{b}}^{s}}, \quad\|a\|_{(m ; k), s}:=\|a\|_{S^{m ; k} H_{\mathrm{b}}^{s}}, \\
\|\mathrm{Op}(a)\|_{m, s}:=\|\mathrm{Op}(a)\|_{\Psi^{m ; 0} H_{\mathrm{b}}^{s}} \equiv\|a\|_{S^{m ; 0} H_{\mathrm{b}}^{s}} \\
\|\mathrm{Op}(a)\|_{(m ; k), s}:=\|\mathrm{Op}(a)\|_{\Psi^{m ; k} H_{\mathrm{b}}^{s}} \equiv\|a\|_{S^{m ; k} H_{\mathrm{b}}^{s}}
\end{gathered}
$$

If $A$ is a b-operator acting on an element of a weighted b-Sobolev space with weight $r$ (which will be apparent from the context), then $\|A\|_{m, s}$ is to be understood as $\left\|x^{-r} A x^{r}\right\|_{m, s}$, similarly for $\|A\|_{(m ; k), s}$. Lastly, for $A \in H_{\mathrm{b}}^{s} \Psi_{\mathrm{b}}^{m}$, i.e. $A$ is a finite sum of products $u_{j} A_{j}$, where $u_{j} \in H_{\mathrm{b}}^{s}, A_{j} \in \Psi_{\mathrm{b}}^{m}$, we write $\|A\|_{H_{\mathrm{b}}^{s} \Psi_{\mathrm{b}}^{m}}$, by an abuse of notation, for an unspecified $H_{\mathrm{b}}^{s} \Psi_{\mathrm{b}}^{m}$-seminorm of $A$. Note that $H_{\mathrm{b}}^{s} \Psi_{\mathrm{b}}^{m} \subset \Psi^{m ; \infty} H_{\mathrm{b}}^{s}$ (with strict inclusion).

Recall the notation $x_{+}=\max (x, 0)$ for $x \in \mathbb{R}$.

Proposition 3.1. (Extension of [30, Proposition 3.9].) Let $s \in \mathbb{R}, A=\mathrm{Op}(a) \in$ $\Psi^{m ; 0} H_{\mathrm{b}}^{s}$, and suppose $s^{\prime} \in \mathbb{R}$ is such that $s \geq s^{\prime}-m, s>n / 2+\left(m-s^{\prime}\right)_{+}$. Then A defines a bounded map $H_{\mathrm{b}}^{s^{\prime}} \rightarrow H_{\mathrm{b}}^{s^{\prime}-m}$, and for all $\mu, \nu$ with

$$
\mu>n / 2+\left(m-s^{\prime}\right)_{+}, \quad \nu>n / 2+\left(m-s^{\prime}\right)_{+}+s^{\prime}-s,
$$

there is a constant $C=C\left(s, s^{\prime}, m, \mu, \nu\right)>0$ such that

$$
\|A u\|_{s^{\prime}-m} \leq C\left(\|A\|_{m, \mu}\|u\|_{s^{\prime}}+\|A\|_{m, s}\|u\|_{\nu}\right)
$$

By the assumptions on $s$ and $s^{\prime}$, one may always choose $\mu=s$ and $\nu=s^{\prime}$ here, which recovers the non-tame estimate given in [30]. Estimates of the form (3.1), called 'tame estimates' e.g. in [28, 46], are crucial for applications in a Nash-Moser iteration scheme, as alluded to in $\S 2$. 
Proof of Proposition 3.1. We compute

$$
\begin{aligned}
\|A u\|_{s^{\prime}-m}^{2} & =\int\langle\zeta\rangle^{2\left(s^{\prime}-m\right)}|\widehat{A u}(\zeta)|^{2} d \zeta \\
& \leq \int\langle\zeta\rangle^{2\left(s^{\prime}-m\right)}\left(\int|\widehat{a}(\zeta-\xi, \xi) \widehat{u}(\xi)| d \xi\right)^{2} d \zeta .
\end{aligned}
$$

We split the inner integral into two pieces, corresponding to the domains of integration $|\zeta-\xi| \leq|\xi|$ and $|\xi| \leq|\zeta-\xi|$, which can be thought of as splitting up the action of $A$ on $u$ into a low-high and a high-low frequency interaction. We estimate

$$
\begin{aligned}
& \int\langle\zeta\rangle^{2\left(s^{\prime}-m\right)}\left(\int_{|\zeta-\xi| \leq|\xi|}|\widehat{a}(\zeta-\xi, \xi) \widehat{u}(\xi)| d \xi\right)^{2} d \zeta \\
& \leq \int\left(\int_{|\zeta-\xi| \leq|\xi|} \frac{\langle\zeta\rangle^{2\left(s^{\prime}-m\right)}\langle\xi\rangle^{2 m}}{\langle\zeta-\xi\rangle^{2 \mu}\langle\xi\rangle^{2 s^{\prime}}} d \xi\right) \\
& \times\left(\int \frac{\langle\zeta-\xi\rangle^{2 \mu}|\widehat{a}(\zeta-\xi, \xi)|^{2}}{\langle\xi\rangle^{2 m}}\langle\xi\rangle^{2 s^{\prime}}|\widehat{u}(\xi)|^{2} d \xi\right) d \zeta,
\end{aligned}
$$

and we claim that the integral which is the first factor on the right hand side is uniformly bounded in $\zeta$ : Indeed, if $s^{\prime}-m \geq 0$, then we use $|\zeta| \leq 2|\xi|$ on the domain of integration, thus

$$
\int_{|\zeta-\xi| \leq|\xi|} \frac{\langle\zeta\rangle^{2\left(s^{\prime}-m\right)}}{\langle\zeta-\xi\rangle^{2 \mu}\langle\xi\rangle^{2\left(s^{\prime}-m\right)}} d \xi \lesssim \int \frac{1}{\langle\zeta-\xi\rangle^{2 \mu}} d \xi \in L_{\zeta}^{\infty}
$$

since $\mu>n / 2$; if, on the other hand, $s^{\prime}-m \leq 0$, then $|\xi| \leq|\zeta-\xi|+|\zeta|$ gives

$$
\int_{|\zeta-\xi| \leq|\xi|} \frac{\langle\xi\rangle^{2\left(m-s^{\prime}\right)}}{\langle\zeta-\xi\rangle^{2 \mu}\langle\zeta\rangle^{2\left(m-s^{\prime}\right)}} d \xi \lesssim \int \frac{1}{\langle\zeta-\xi\rangle^{2\left(\mu-\left(m-s^{\prime}\right)\right)}}+\frac{1}{\langle\zeta-\xi\rangle^{2 \mu}} d \xi \in L_{\zeta}^{\infty},
$$

since $\mu>n / 2+\left(m-s^{\prime}\right)$; hence, from (3.2), the $H_{\mathrm{b}}^{s^{\prime}-m}$ norm of the low-high frequency interaction in $A u$ is bounded by $C_{\mu}\|a\|_{m, \mu}\|u\|_{s^{\prime}}$.

We estimate the norm of high-low interaction in a similar way: We have

$$
\begin{aligned}
\int\langle\zeta\rangle^{2\left(s^{\prime}-m\right)}\left(\int_{|\xi| \leq|\zeta-\xi|}|\widehat{a}(\zeta-\xi, \xi) \widehat{u}(\xi)| d \xi\right)^{2} d \zeta \\
\leq \int\left(\int_{|\xi| \leq|\zeta-\xi|} \frac{\langle\zeta\rangle^{2\left(s^{\prime}-m\right)}\langle\xi\rangle^{2 m}}{\langle\zeta-\xi\rangle^{2 s}\langle\xi\rangle^{2 \nu}} d \xi\right) \\
\quad \times\left(\int \frac{\langle\zeta-\xi\rangle^{2 s}|\widehat{a}(\zeta-\xi, \xi)|^{2}}{\langle\xi\rangle^{2 m}}\langle\xi\rangle^{2 \nu}|\widehat{u}(\xi)|^{2} d \xi\right) d \zeta .
\end{aligned}
$$

If $s^{\prime}-m \geq 0$, the first inner integral on the right hand side is bounded by

$$
\int_{|\xi| \leq|\zeta-\xi|} \frac{1}{\langle\zeta-\xi\rangle^{2\left(s-s^{\prime}+m\right)}\langle\xi\rangle^{2(\nu-m)}} d \xi \leq \int \frac{1}{\langle\xi\rangle^{2\left(s-s^{\prime}+\nu\right)}} d \xi
$$

where we use $s \geq s^{\prime}-m$, and this integral is finite in view of $\nu>n / 2+s^{\prime}-s$; if $s^{\prime}-m \leq 0$, then

$$
\int_{|\xi| \leq|\zeta-\xi|} \frac{1}{\langle\zeta\rangle^{2\left(m-s^{\prime}\right)}\langle\zeta-\xi\rangle^{2 s}\langle\xi\rangle^{2(\nu-m)}} d \xi \leq \int \frac{1}{\langle\xi\rangle^{2(\nu-m+s)}} d \xi
$$


which is finite in view of $\nu>n / 2+m-s$. In summary, we need $\nu>n / 2+$ $\max \left(m, s^{\prime}\right)-s=n / 2+\left(m-s^{\prime}\right)_{+}+s^{\prime}-s$ and can then bound the $H_{\mathrm{b}}^{s^{\prime}-m}$ norm of the high-low interaction by $C_{\nu}\|a\|_{m, s}\|u\|_{\nu}$. The proof is complete.

Using $H_{\mathrm{b}}^{s} \subset S^{0 ; 0} H_{\mathrm{b}}^{s}$, we obtain the following weak version of the Moser estimate for the product of two b-Sobolev functions:

Corollary 3.2. Let $s>n / 2,\left|s^{\prime}\right| \leq s$. If $u \in H_{\mathrm{b}}^{s}, v \in H_{\mathrm{b}}^{s^{\prime}}$, then $u v \in H_{\mathrm{b}}^{s^{\prime}}$, and one has an estimate

$$
\|u v\|_{s^{\prime}} \leq C\left(\|u\|_{\mu}\|v\|_{s^{\prime}}+\|u\|_{s}\|v\|_{\nu}\right)
$$

for all $\mu>n / 2+\left(-s^{\prime}\right)_{+}, \nu>n / 2+s_{+}^{\prime}-s$, where $C=C\left(s, s^{\prime}, \mu, \nu\right)$. In particular, for $u, v \in H_{\mathrm{b}}^{s}$,

for $\mu>n / 2$, with $C=C(s, \mu)$.

$$
\|u v\|_{s} \leq C\left(\|u\|_{\mu}\|v\|_{s}+\|u\|_{s}\|v\|_{\mu}\right)
$$

3.2. Operator compositions. We give a tame estimate for the norms of expansion and remainder terms arising in the composition of two non-smooth operators:

Proposition 3.3. Suppose $s, m, m^{\prime} \in \mathbb{R}, k, k^{\prime} \in \mathbb{N}_{0}$ are such that $s>n / 2, s \leq s^{\prime}-k$ and $k \geq m+k^{\prime}$. Suppose $P=p\left(z,{ }^{\mathrm{b}} D\right) \in \Psi^{m ; k} H_{\mathrm{b}}^{s}, Q=q\left(z,{ }^{\mathrm{b}} D\right) \in \Psi^{m^{\prime} ; 0} H_{\mathrm{b}}^{s^{\prime}}$. Put

$$
\begin{gathered}
E_{j}:=\sum_{|\beta|=j} \frac{1}{\beta !}\left(\partial_{\zeta}^{\beta} p^{\mathrm{b}} D_{z}^{\beta} q\right)\left(z,{ }^{\mathrm{b}} D\right), \\
R:=P \circ Q-\sum_{0 \leq j<k} E_{j} .
\end{gathered}
$$

Then $E_{j} \in \Psi^{m+m^{\prime}-j ; 0} H_{\mathrm{b}}^{s}$ and $R \in \Psi^{m^{\prime}-k^{\prime} ; 0} H_{\mathrm{b}}^{s}$, and for $\mu>n / 2$ fixed,

$$
\begin{gathered}
\left\|E_{j}\right\|_{m+m^{\prime}-j, s} \leq C\left(\|P\|_{(m ; j), \mu}\|Q\|_{m, s+j}+\|P\|_{(m ; j), s}\|Q\|_{m^{\prime}, \mu+j}\right), \\
\|R\|_{m^{\prime}-k^{\prime}, s} \leq C\left(\|P\|_{(m ; k), \mu}\|Q\|_{m^{\prime}, s+k}+\|P\|_{(m ; k), s}\|Q\|_{m^{\prime}, \mu+k}\right) .
\end{gathered}
$$

Proof. The statements about the $E_{j}$ follow from Corollary 3.2. For the purpose of proving the estimate for $R$, we define

$$
p_{0}=\partial_{\zeta}^{k} p \in S^{m-k ; 0} H_{\mathrm{b}}^{s}, \quad{ }^{\mathrm{b}} D_{z}^{k} q \in S^{m^{\prime} ; 0} H_{\mathrm{b}}^{s^{\prime}-k},
$$

where we write $\partial_{\zeta}^{k}=\left(\partial_{\zeta}^{\beta}\right)_{|\beta|=k}$, similarly for ${ }^{\mathrm{b}} D_{z}^{k}$. Notice that in particular $p_{0} \in$ $S^{0 ; 0} H_{\mathrm{b}}^{s}$. Then $R=r\left(z,{ }^{\mathrm{b}} D\right)$ with

$$
|\widehat{r}(\eta ; \zeta)| \lesssim \int\left(\int_{0}^{1} p_{0}(\eta-\xi ; \zeta+t \xi) d t\right) q_{0}(\xi ; \zeta) d \xi
$$

by Taylor's formula, hence

$$
\begin{aligned}
& \int \frac{\langle\eta\rangle^{2 s}|\widehat{r}(\eta ; \zeta)|^{2}}{\langle\zeta\rangle^{2 m^{\prime}}} d \eta \\
& \quad \lesssim \int\left(\int_{|\eta-\xi| \leq|\xi|} \frac{\langle\eta\rangle^{2 s}}{\langle\eta-\xi\rangle^{2 \mu}\langle\xi\rangle^{2 s}} d \xi\right) \\
& \quad \times\left(\int\left(\int_{0}^{1}\langle\eta-\xi\rangle^{2 \mu}\left|p_{0}(\eta-\xi, \zeta+t \xi)\right|^{2} d t\right) \frac{\langle\xi\rangle^{2 s}\left|q_{0}(\xi ; \zeta)\right|^{2}}{\langle\zeta\rangle^{2 m^{\prime}}} d \xi\right) d \eta \\
& \quad+\int\left(\int_{|\xi| \leq|\eta-\xi|} \frac{\langle\eta\rangle^{2 s}}{\langle\eta-\xi\rangle^{2 s}\langle\xi\rangle^{2 \mu}} d \xi\right)
\end{aligned}
$$




$$
\times\left(\int\left(\int_{0}^{1}\langle\eta-\xi\rangle^{2 s}\left|p_{0}(\eta-\xi, \zeta+t \xi)\right|^{2} d t\right) \frac{\langle\xi\rangle^{2 \mu}\left|q_{0}(\xi ; \zeta)\right|^{2}}{\langle\zeta\rangle^{2 m^{\prime}}} d \xi\right) d \eta
$$

which implies the claimed estimate for $k^{\prime}=0$. For $k^{\prime}>0$, we use a trick of Beals and Reed [4] as in the proof of Theorem 3.12 in [30] to reduce the statement to the case $k^{\prime}=0$ : Recall that the idea is to split up $q(z, \zeta)$ into a 'trivial' part $q_{0}$ with compact support in $\zeta$ and $n$ parts $q_{i}$, where $q_{i}$ has support in $\left|\zeta_{i}\right| \geq 1$, and then writing

$$
P \circ Q_{i}=\sum_{j=0}^{k^{\prime}} c_{j k^{\prime}} P^{\mathrm{b}} D_{z_{i}}^{k^{\prime}-j} \circ\left({ }^{\mathrm{b}} D_{z_{i}}^{j} q_{i}\right)\left(z,{ }^{\mathrm{b}} D\right)^{\mathrm{b}} D_{z_{i}}^{-k^{\prime}}
$$

for some constants $c_{j k^{\prime}} \in \mathbb{R}$ using the Leibniz rule; then what we have proved above for $k^{\prime}=0$ can be applied to the $j$-th summand on the right hand side, which we expand to order $k-j$, giving the result.

3.3. Reciprocals of and compositions with $H_{\mathrm{b}}^{s}$ functions. We also need sharper bounds for reciprocals and compositions of b-Sobolev functions on a compact $n$-dimensional manifold with boundary. Localizing using a partition of unity, we can simply work on $\overline{\mathbb{R}_{+}^{n}}$.

Proposition 3.4. (Extension of [30, Lemma 4.1].) Let $s>n / 2+1, u, w \in H_{\mathrm{b}}^{s}$, $a \in \mathcal{C}^{\infty}$, and suppose that $|a+u| \geq c_{0}$ near $\operatorname{supp} w$. Then $w /(a+u) \in H_{\mathrm{b}}^{s}$, and one has an estimate

$$
\left\|\frac{w}{a+u}\right\|_{s} \leq C\left(\|u\|_{\mu},\|a\|_{C^{N}}\right) c_{0}^{-1} \max \left(c_{0}^{-\lceil s\rceil}, 1\right)\left(\|w\|_{s}+\|w\|_{\mu}\left(1+\|u\|_{s}\right)\right) .
$$

for any fixed $\mu>n / 2+1$ and some s-dependent $N \in \mathbb{N}$.

Proof. Choose $\psi_{0}, \psi \in \mathcal{C}^{\infty}$ such that $\psi_{0} \equiv 1$ on $\operatorname{supp} w, \psi \equiv 1$ on $\operatorname{supp} \psi_{0}$, and such that moreover $|a+u| \geq c_{0}>0$ on $\operatorname{supp} \psi$. Then we have $\|w /(a+u)\|_{0} \leq c_{0}^{-1}\|w\|_{0}$. We now iteratively prove higher regularity of $w /(a+u)$ and an accompanying 'tame' estimate: Let us assume $w /(a+u) \in H_{\mathrm{b}}^{s^{\prime}-1}$ for some $1 \leq s^{\prime} \leq s$. Let $\Lambda_{s^{\prime}}=\lambda_{s^{\prime}}\left({ }^{\mathrm{b}} D\right) \in \Psi_{\mathrm{b}}^{s^{\prime}}$ be an operator with principal symbol $\langle\zeta\rangle^{s^{\prime}}$. Then

$$
\begin{aligned}
\| \Lambda_{s^{\prime}} & \frac{w}{a+u}\left\|_{0} \leq\right\|(1-\psi) \Lambda_{s^{\prime}} \frac{\psi_{0} w}{a+u}\left\|_{0}+\right\| \psi \Lambda_{s^{\prime}} \frac{\psi_{0} w}{a+u} \|_{0} \\
& \lesssim\left\|\frac{w}{a+u}\right\|_{0}+c_{0}^{-1}\left\|\psi(a+u) \Lambda_{s^{\prime}} \frac{w}{a+u}\right\|_{0} \\
& \leq c_{0}^{-1}\|w\|_{0}+c_{0}^{-1}\left(\left\|\psi \Lambda_{s^{\prime}} w\right\|_{0}+\left\|\psi\left[\Lambda_{s^{\prime}}, a+u\right] \frac{w}{a+u}\right\|_{0}\right) \\
& \lesssim c_{0}^{-1}\left(\|w\|_{s^{\prime}}+\left\|\frac{w}{a+u}\right\|_{s^{\prime}-1}+\left\|\psi\left[\Lambda_{s^{\prime}}, u\right] \frac{w}{a+u}\right\|_{0}\right),
\end{aligned}
$$

where we used that the support assumptions on $\psi_{0}$ and $\psi$ imply $(1-\psi) \Lambda_{s^{\prime}} \psi_{0} \in$ $\Psi_{\mathrm{b}}^{-\infty}$, and $\psi\left[\Lambda_{s^{\prime}}, a\right] \in \Psi_{\mathrm{b}}^{s^{\prime}-1}$. Hence, in order to prove that $w /(a+u) \in H_{\mathrm{b}}^{s^{\prime}}$, it suffices to show that $\left[\Lambda_{s^{\prime}}, u\right]: H_{\mathrm{b}}^{s^{\prime}-1} \rightarrow H_{\mathrm{b}}^{0}$. Let $v \in H_{\mathrm{b}}^{s^{\prime}-1}$. Since

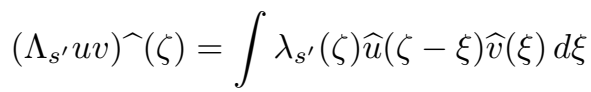

$$
\begin{aligned}
& \left(u \Lambda_{s^{\prime}} v\right)^{\wedge}(\zeta)=\int \widehat{u}(\zeta-\xi) \lambda_{s^{\prime}}(\xi) \widehat{v}(\xi) d \xi,
\end{aligned}
$$


we have, by taking a first order Taylor expansion of $\lambda_{s^{\prime}}(\zeta)=\lambda_{s^{\prime}}(\xi+(\zeta-\xi))$ around $\zeta=\xi$

$$
\left(\left[\Lambda_{s^{\prime}}, u\right] v\right)^{\wedge}(\zeta)=\sum_{|\beta|=1} \int\left(\int_{0}^{1} \partial_{\zeta}^{\beta} \lambda_{s^{\prime}}(\xi+t(\zeta-\xi)) d t\right)\left({ }^{\mathrm{b}} D_{z}^{\beta} u\right)^{\wedge}(\zeta-\xi) \widehat{v}(\xi) d \xi,
$$

thus, writing $u^{\prime}={ }^{\mathrm{b}} D_{z} u \in H_{\mathrm{b}}^{s-1}$,

$$
\left|\left(\left[\Lambda_{s^{\prime}}, u\right] v\right)^{\wedge}(\zeta)\right| \lesssim \int\left(\int_{0}^{1}\langle\xi+t(\zeta-\xi)\rangle^{s^{\prime}-1} d t\right)\left|\widehat{u^{\prime}}(\zeta-\xi)\right||\widehat{v}(\xi)| d \xi .
$$

To obtain a tame estimate for the $L_{\zeta}^{2}$ norm of this expression, we again use the method of decomposing the integral into low-high and high-low components: The low-high component is bounded by

$$
\begin{aligned}
& \int\left(\int_{|\zeta-\xi| \leq|\xi|} \frac{\sup _{0 \leq t \leq 1}\langle\xi+t(\zeta-\xi)\rangle^{2\left(s^{\prime}-1\right)}}{\langle\zeta-\xi\rangle^{2(\mu-1)}\langle\xi\rangle^{2\left(s^{\prime}-1\right)}} d \xi\right) \\
& \times\left(\int\langle\zeta-\xi\rangle^{2(\mu-1)}\left|\widehat{u}^{\prime}(\zeta-\xi)\right|^{2}\langle\xi\rangle^{2\left(s^{\prime}-1\right)}|\widehat{v}(\xi)|^{2} d \xi\right) d \zeta ;
\end{aligned}
$$

the first inner integral, in view of $s^{\prime} \geq 1$, so the sup is bounded by $\langle\xi\rangle^{2\left(s^{\prime}-1\right)}$, which cancels the corresponding term in the denominator, is finite for $\mu>n / 2+1$. For the high-low component, we likewise estimate

$$
\begin{aligned}
& \int\left(\int_{|\xi| \leq|\zeta-\xi|} \frac{\sup _{0 \leq t \leq 1}\langle\xi+t(\zeta-\xi)\rangle^{2\left(s^{\prime}-1\right)}}{\langle\zeta-\xi\rangle^{2(s-1)}\langle\xi\rangle^{2 \nu}} d \xi\right) \\
& \times\left(\int\langle\zeta-\xi\rangle^{2 s}\left|\widehat{u^{\prime}}(\zeta-\xi)\right|^{2}\langle\xi\rangle^{2 \nu}|\widehat{v}(\xi)|^{2} d \xi\right) d \zeta,
\end{aligned}
$$

and the first inner integral on the right hand side is bounded by

$$
\int_{|\xi| \leq|\zeta-\xi|} \frac{1}{\langle\zeta-\xi\rangle^{2\left(s-s^{\prime}\right)}\langle\xi\rangle^{2 \nu}} d \xi \leq \int \frac{1}{\langle\xi\rangle^{2\left(s-s^{\prime}+\nu\right)}} d \xi
$$

because of $s \geq s^{\prime}$, which is finite for $\nu>n / 2+s^{\prime}-s$. We conclude that

$$
\left\|\left[\Lambda_{s^{\prime}}, u\right] v\right\|_{0} \leq C_{\mu \nu}\left(\|u\|_{\mu}\|v\|_{s^{\prime}-1}+\|u\|_{s^{\prime}}\|v\|_{\nu}\right)
$$

for $\mu>n / 2+1, \nu>n / 2+s^{\prime}-s$. Plugging this into (3.5) yields

$$
\left\|\frac{w}{a+u}\right\|_{s^{\prime}} \lesssim c_{0}^{-1}\left(\|w\|_{s^{\prime}}+\left(1+\|u\|_{\mu}\right)\left\|\frac{w}{a+u}\right\|_{s^{\prime}-1}+\|u\|_{s^{\prime}}\left\|\frac{w}{a+u}\right\|_{\nu}\right),
$$

where the implicit constant in the inequality is independent of $c_{0}, w$ and $u$. Using the abbreviations $q_{\sigma}:=\|w /(a+u)\|_{\sigma}, u_{\sigma}=\|u\|_{\sigma}, w_{\sigma}=\|w\|_{\sigma}$ and fixing $\mu>n / 2+1$, this means

$$
q_{s^{\prime}} \lesssim c_{0}^{-1}\left(w_{s^{\prime}}+\left(1+u_{\mu}\right) q_{s^{\prime}-1}+u_{s^{\prime}} q_{\nu}\right), \quad \nu>n / 2+s^{\prime}-s,
$$

with the implicit constant being independent of $c_{0}, w, a, u, \mu$. We will use this for $s^{\prime} \leq \gamma:=\lfloor n / 2\rfloor+1$ with $\nu=s^{\prime}-1$, and for $s^{\prime}>\gamma$, we will take $\nu=\gamma$, thus obtaining a tame estimate for $q_{s}$. In more detail, for $1 \leq s^{\prime} \leq \gamma$, we have

$$
q_{s^{\prime}} \lesssim c_{0}^{-1}\left(w_{s^{\prime}}+\left(1+u_{s^{\prime}}\right) q_{s^{\prime}-1}\right)
$$


which gives, with $C_{0}=\max \left(1, c_{0}^{-1}\right)$,

$$
q_{\gamma} \lesssim c_{0}^{-1} w_{\gamma} \sum_{j=0}^{\gamma-1}\left(c_{0}^{-1}\left(1+u_{\gamma}\right)\right)^{j}+\left(c_{0}^{-1}\left(1+u_{\gamma}\right)\right)^{\gamma} q_{0} \lesssim c_{0}^{-1} C_{0}^{\gamma} w_{\gamma}\left(1+u_{\gamma}\right)^{\gamma}
$$

using the bound $q_{0} \leq c_{0}^{-1} w_{0} \leq c_{0}^{-1} w_{\gamma}$. For $\gamma<s^{\prime} \leq s$, we have

$$
q_{s^{\prime}} \lesssim c_{0}^{-1}\left(w_{s}+u_{s} q_{\gamma}+\left(1+u_{\mu}\right) q_{s^{\prime}-1}\right),
$$

thus for integer $k \geq 1$ with $\gamma+k \leq s$,

$$
\begin{aligned}
q_{\gamma+k} & \leq c_{0}^{-1}\left(w_{s}+u_{s} q_{\gamma}\right) \sum_{j=0}^{k-1}\left(c_{0}^{-1}\left(1+u_{\mu}\right)\right)^{j}+\left(c_{0}^{-1}\left(1+u_{\mu}\right)\right)^{k} q_{\gamma} \\
& \lesssim c_{0}^{-1} C_{0}^{k-1}\left(1+u_{\mu}\right)^{k}\left(w_{s}+\left(1+u_{s}\right) q_{\gamma}\right) \\
& \lesssim c_{0}^{-1} C_{0}^{\gamma+k}\left(1+u_{\mu}\right)^{\gamma+k}\left(w_{s}+\left(1+u_{s}\right) w_{\gamma}\right),
\end{aligned}
$$

where we used $\mu>\gamma$ in the last inequality, thus proving the estimate (3.4) in case $s$ is an integer; in the general case, we just use $q_{\gamma^{\prime}} \leq q_{\gamma}$ for $\gamma^{\prime}<\gamma$, in particular for $\gamma^{\prime}=s-\lceil s-\gamma\rceil$, and use the above with $q_{\gamma+k}$ replaced by $q_{\gamma^{\prime}+k}$.

As in [30], one thus obtains regularity results for compositions, but now with sharper estimates. To illustrate how to obtain these, let us prove an extension of [30, Proposition 4.5]. Let $M$ be a compact $n$-dimensional manifold with boundary, $s>n / 2+1, \alpha \geq 0$.

Proposition 3.5. Let $u \in H_{\mathrm{b}}^{s, \alpha}(M)$. If $F: \Omega \rightarrow \mathbb{C}, F(0)=0$, is holomorphic in a simply connected neighborhood $\Omega$ of the range of $u$, then $F(u) \in H_{\mathrm{b}}^{s, \alpha}(M)$, and

$$
\|F(u)\|_{s, \alpha} \leq C\left(\|u\|_{\mu, \alpha}\right)\left(1+\|u\|_{s, \alpha}\right)
$$

for fixed $\mu>n / 2+1$. Moreover, there exists $\epsilon>0$ such that $F(v) \in H_{\mathrm{b}}^{s, \alpha}(M)$ depends continuously on $v \in H_{\mathrm{b}}^{s, \alpha}(M),\|u-v\|_{s, \alpha}<\epsilon$.

Proof. Observe that $u(M)$ is compact. Let $\gamma \subset \mathbb{C}$ denote a smooth contour which is disjoint from $u(M)$, has winding number 1 around every point in $u(M)$, and lies within the region of holomorphicity of $F$. Then, writing $F(z)=z F_{1}(z)$ with $F_{1}$ holomorphic in $\Omega$, we have

$$
F(u)=\frac{1}{2 \pi i} \oint_{\gamma} F_{1}(\zeta) \frac{u}{\zeta-u} d \zeta
$$

Since $\gamma \ni \zeta \mapsto u /(\zeta-u) \in H_{\mathrm{b}}^{s, \alpha}(M)$ is continuous by Proposition 3.4, we obtain, using the estimate (3.4),

$$
\|F(u)\|_{s, \alpha} \leq C\left(\|u\|_{\mu}\right)\left(\|u\|_{s, \alpha}+\|u\|_{\mu, \alpha}\left(1+\|u\|_{s}\right)\right)
$$

which implies (3.6) in view of $\alpha \geq 0$. The continuous (in fact, Lipschitz) dependence of $F(v)$ on $v$ is a consequence of Proposition 3.4 and Corollary 3.2.

We also study compositions $F(u)$ for $F \in \mathcal{C}^{\infty}(\mathbb{R} ; \mathbb{C})$ and real-valued $u$.

Proposition 3.6. (Extension of [30, Proposition 4.7].) Let $F \in \mathcal{C}^{\infty}(\mathbb{R} ; \mathbb{C}), F(0)=$ 0 . Then for $u \in H_{\mathrm{b}}^{s, \alpha}(M ; \mathbb{R})$, we have $F(u) \in H_{\mathrm{b}}^{s, \alpha}(M)$, and one has an estimate

$$
\|F(u)\|_{s, \alpha} \leq C\left(\|u\|_{\mu, \alpha}\right)\left(1+\|u\|_{s, \alpha}\right)
$$

for fixed $\mu>n / 2+1$. In fact, $F(u)$ depends continuously on $u$ in the same sense as in Proposition 3.5. 
Proof. The proof is the same as in [30], using almost analytic extensions, only we now use the sharper estimate (3.4) to obtain (3.7).

Proposition 3.7. (Extension of [30, Proposition 4.8].) Let $F \in \mathcal{C}^{\infty}(\mathbb{R} ; \mathbb{C})$, and $u^{\prime} \in \mathcal{C}^{\infty}(M ; \mathbb{R}), u^{\prime \prime} \in H_{\mathrm{b}}^{s, \alpha}(M ; \mathbb{R}) ;$ put $u=u^{\prime}+u^{\prime \prime}$. Then $F(u) \in \mathcal{C}^{\infty}(M)+$ $H_{\mathrm{b}}^{s, \alpha}(M)$, and one has an estimate

$$
\left\|F(u)-F\left(u^{\prime}\right)\right\|_{s, \alpha} \leq C\left(\left\|u^{\prime}\right\|_{C^{N}},\left\|u^{\prime \prime}\right\|_{\mu, \alpha}\right)\left(1+\left\|u^{\prime \prime}\right\|_{s, \alpha}\right)
$$

for fixed $\mu>n / 2+1$ and some $N \in \mathbb{N}$. In fact, $F(u)$ depends continuously on $u$.

Proof. The proof is the same as in [30], but now uses the sharper estimate (3.4).

\section{Microlocal Regularity: tame estimates}

When stating microlocal regularity estimates (like elliptic regularity, real principal type propagation, etc.) for operators with coefficients in $H_{\mathrm{b}}^{s}\left(\overline{\mathbb{R}_{+}^{n}}\right)$, we will give two quantitative statements, one for 'low' regularities $\sigma \lesssim n / 2$, in which we will not make use of any tame estimates established earlier, and one for 'high' regularities $n / 2 \lesssim \sigma \lesssim s$, in which the tame estimates will be used.

To concisely write down tame estimates, we use the following notation: The right hand side of a tame estimate will be a real-valued function, denoted by $L$, of the form

$$
\begin{aligned}
L\left(p_{1}^{\ell}, \ldots, p_{a}^{\ell} ; p_{1}^{h}, \ldots, p_{b}^{h} ; u_{1}^{\ell}, \ldots, u_{c}^{\ell} ; u_{1}^{h}, \ldots, u_{d}^{h}\right) & \\
& =\sum_{j=1}^{d} c_{j}\left(p_{1}^{\ell}, \ldots, p_{a}^{\ell}\right) u_{j}^{h}+\sum_{j=1}^{b} \sum_{k=1}^{c} c_{j k}\left(p_{1}^{\ell}, \ldots, p_{a}^{\ell}\right) p_{j}^{h} u_{k}^{\ell}
\end{aligned}
$$

here, the $c_{j}$ and $c_{j k}$ are continuous functions. In applications, $p_{j}^{\ell / h}$ will be a low/high regularity norm of the coefficients of a non-smooth operator, and $u_{j}^{\ell / h}$ will be a low/high regularity norm of a function that an operator is applied to. The important feature of such functions $L$ is that they are linear in the $u_{j}^{\ell / h}$, and all $p_{j}^{h}, u_{j}^{h}$, corresponding to high regularity norms, only appear in the first power.

4.1. Elliptic regularity. Concretely, we have the following quantitative microlocal elliptic estimate, which will be used to control solutions to equations $P u=f$ microlocally away from the characteristic set of $P$.

Proposition 4.1. (Cf. [30, Theorem 5.1].) Let $m, s, r \in \mathbb{R}$ and $\zeta_{0} \in{ }^{\mathrm{b}} S^{*} \overline{\mathbb{R}_{+}^{n}}$. Suppose $P^{\prime}=p^{\prime}\left(z,{ }^{\mathrm{b}} D\right) \in H_{\mathrm{b}}^{s} \Psi_{\mathrm{b}}^{m}\left(\overline{\mathbb{R}_{+}^{n}}\right)$ has a homogeneous principal symbol $p_{m}^{\prime}$. Moreover, let $R \in \Psi_{\mathrm{b}}^{m-1 ; 0} H_{\mathrm{b}}^{s-1}\left(\overline{\mathbb{R}_{+}^{n}}\right)$. Let $P=P^{\prime}+R$, and suppose $p_{m} \equiv p_{m}^{\prime}$ is elliptic at $\zeta_{0}$. Then there exists $B \in \Psi_{\mathrm{b}}^{0}\left(\overline{\mathbb{R}_{+}^{n}}\right)$ elliptic at $\zeta_{0}$ such that the following holds: Let $\widetilde{s} \in \mathbb{R}$ be such that $\widetilde{s} \leq s-1$ and $s>n / 2+1+(-\widetilde{s})_{+}$, and suppose that $u \in H_{\mathrm{b}}^{\widetilde{s}+m-1, r}\left(\overline{\mathbb{R}_{+}^{n}}\right)$ satisfies

$$
P u=f \in H_{\mathrm{b}}^{\widetilde{s}, r}\left(\overline{\mathbb{R}_{+}^{n}}\right) .
$$

Then $B u \in H_{\mathrm{b}}^{\widetilde{s}+m}$, and for $\widetilde{s} \leq n / 2+t, t>0$, the estimate

$$
\begin{gathered}
\|B u\|_{\widetilde{s}+m, r} \leq C\left(\left\|P^{\prime}\right\|_{(m ; 1), n / 2+1+(-\widetilde{s})_{+}+t},\|R\|_{m-1, n / 2+(-\widetilde{s})_{+}+t}\right) \\
\times\left(\|u\|_{\widetilde{s}+m-1, r}+\|f\|_{\widetilde{s}, r}\right)
\end{gathered}
$$


holds; recall here that $P^{\prime} \in H_{\mathrm{b}}^{s} \Psi_{\mathrm{b}}^{m} \subset \Psi^{m ; \infty} H_{\mathrm{b}}^{s}$, justifying the use of the norm on $P^{\prime}$ in this estimate. For $\widetilde{s}>n / 2, \epsilon>0$, there is a tame estimate

$$
\begin{array}{r}
\|B u\|_{\widetilde{s}+m, r} \leq L\left(\left\|P^{\prime}\right\|_{(m ; 1), n / 2+1+\epsilon},\|R\|_{m-1, n / 2+\epsilon} ;\left\|P^{\prime}\right\|_{(m ; 1), s},\|R\|_{m-1, s-1} ;\right. \\
\left.\|u\|_{n / 2+m-1+\epsilon, r},\|f\|_{n / 2-1+\epsilon, r} ;\|u\|_{\widetilde{s}+m-1, r},\|f\|_{\widetilde{s}, r}\right) .
\end{array}
$$

Let us point out that in our application of such an estimate to the study of nonlinear equations it will be irrelevant what exactly the low regularity norms in (4.3) are; in fact, it will be sufficient to know that there is some tame estimate of the general form (4.3), and this in turn is in fact clear without any computation, namely it follows directly from the fact that we have tame estimates for all 'nonsmooth' operations involved in the proof of this proposition. Thus, a simpler, but less precise, statement of Proposition 4.1 is the following: Let $P \in H_{\mathrm{b}}^{s} \Psi_{\mathrm{b}}^{m}$ (we ignore $R$ for brevity) be elliptic at $\zeta_{0}$, then for sufficiently large $d>0$, and for $s, \widetilde{s}$ with $s \geq \widetilde{s}+d, \widetilde{s} \geq d$, one has an estimate

$$
\|B u\|_{\widetilde{s}+m} \leq C\left(\|P\|_{(m ; 1), d}\right)\left(\|u\|_{\widetilde{s}+m-1}+\|f\|_{\widetilde{s}}+\|P\|_{(m ; 1), s}\left(\|u\|_{d}+\|f\|_{d}\right)\right),
$$

likewise with the weight $r$ added. Similar remarks apply to all further tame microlocal regularity results below. The only point where the precise numerology does matter is when one wants to find an explicit bound on the number of required derivatives for the forcing term in Theorems 2, 3 and 4, as we will do.

Proof of Proposition 4.1. We can assume that $r=0$ by conjugating $P$ by $x^{-r}$. Choose $a_{0} \in S^{0}$ elliptic at $\zeta_{0}$ such that $p_{m}$ is elliptic (and non-vanishing, which only matters near the zero section) on $\operatorname{supp} a_{0}$. Let $\Lambda_{m} \in \Psi_{\mathrm{b}}^{m}$ be a b-ps.d.o with full symbol $\lambda_{m}(\zeta)$ independent of $z$, whose principal symbol is $\langle\zeta\rangle^{m}$, and define

$$
q(z, \zeta):=a_{0}(z, \zeta) \lambda_{m}(\zeta) / p_{m}(z, \zeta) \in S^{0 ; \infty} H_{\mathrm{b}}^{s}, \quad Q=q\left(z,{ }^{\mathrm{b}} D\right)
$$

then by Proposition 3.4 and Corollary 3.2, we have

$$
\|Q\|_{(0 ; k), \sigma} \leq C\left(\left\|P^{\prime}\right\|_{(m ; k), n / 2+1+\epsilon}\right)\left(1+\left\|P^{\prime}\right\|_{(m ; k), \sigma}\right), \quad \sigma>n / 2+1, \epsilon>0 .
$$

Put $B=a_{0}\left(z,{ }^{\mathrm{b}} D\right) \Lambda_{m}$, then

$$
Q \circ P^{\prime}=B+R^{\prime}
$$

with $R^{\prime} \in \Psi^{m-1 ; 0} H_{\mathrm{b}}^{s-1}$; by Proposition 3.3, we have for $n / 2<\sigma \leq s-1$

$$
\left\|R^{\prime}\right\|_{m-1, \sigma} \lesssim\|Q\|_{(0 ; 1), \mu}\left\|P^{\prime}\right\|_{(m ; 1), \sigma+1}+\|Q\|_{(0 ; 1), \sigma}\left\|P^{\prime}\right\|_{(m ; 1), \mu+1}, \quad \mu>n / 2 .
$$

Now, since $B u=Q P^{\prime} u-R^{\prime} u=Q f-Q R u-R^{\prime} u$, we need to estimate the $H_{\mathrm{b}}^{\widetilde{s}}$-norms of $Q f, Q R u$ and $R^{\prime} u$, which we will do using Proposition 3.1. In the low regularity regime, we have, for $t>0$ and $\widetilde{s} \leq n / 2+t$, using (4.4) and (4.5):

$$
\begin{aligned}
&\|Q f\|_{\widetilde{s}} \lesssim\|Q\|_{0, n / 2+(-\widetilde{s})_{+}+t}\|f\|_{\widetilde{s}} \leq C\left(\left\|P^{\prime}\right\|_{m, n / 2+1+(-\widetilde{s})_{+}+t}\right)\|f\|_{\widetilde{s}} \\
&\left\|R^{\prime} u\right\|_{\widetilde{s}} \lesssim\left\|R^{\prime}\right\|_{m-1, n / 2+(-\widetilde{s})_{+}+t}\|u\|_{\widetilde{s}+m-1} \\
& \quad \leq C\left(\left\|P^{\prime}\right\|_{(m ; 1), n / 2+1+(-\widetilde{s})_{+}+t}\right)\|u\|_{\widetilde{s}+m-1}, \\
&\|Q R u\|_{\widetilde{s}} \leq C\left(\left\|P^{\prime}\right\|_{m, n / 2+1+(-\widetilde{s})_{+}+t}\right)\|R\|_{m-1, n / 2+(-\widetilde{s})_{+}+t}\|u\|_{\widetilde{s}+m-1},
\end{aligned}
$$

giving (4.2). In the high regularity regime, in fact for $0 \leq \widetilde{s} \leq s-1$, we have, for $\epsilon>0$

$$
\begin{aligned}
\|Q f\|_{\tilde{s}} \lesssim\|Q\|_{0, n / 2+\epsilon}\|f\|_{\widetilde{s}}+\|Q\|_{0, s}\|f\|_{n / 2-1+\epsilon} \\
\quad \leq C\left(\left\|P^{\prime}\right\|_{m, n / 2+1+\epsilon}\right)\left(\|f\|_{\widetilde{s}}+\left(1+\left\|P^{\prime}\right\|_{m, s}\right)\|f\|_{n / 2-1+\epsilon}\right)
\end{aligned}
$$




$$
\begin{aligned}
&\left\|R^{\prime} u\right\|_{\tilde{s}} \lesssim\left\|R^{\prime}\right\|_{m-1, n / 2+\epsilon}\|u\|_{\tilde{s}+m-1}+\left\|R^{\prime}\right\|_{m-1, s-1}\|u\|_{n / 2+m-1+\epsilon} \\
& \leq C\left(\left\|P^{\prime}\right\|_{(m ; 1), n / 2+1+\epsilon}\right)\left(\|u\|_{\widetilde{s}+m-1}+\left(1+\left\|P^{\prime}\right\|_{(m ; 1), s}\right)\|u\|_{n / 2+m-1+\epsilon}\right), \\
&\|Q R u\|_{\widetilde{s}} \leq L\left(\left\|P^{\prime}\right\|_{m, n / 2+1+\epsilon},\|R\|_{m-1, n / 2+\epsilon} ;\left\|P^{\prime}\right\|_{m, s},\|R\|_{m-1, s-1} ;\right. \\
&\left.\|u\|_{n / 2+m-1+\epsilon} ;\|u\|_{\tilde{s}+m-1}\right)
\end{aligned}
$$

giving (4.3). The proof is complete.

There is a similar tame microlocal elliptic estimate for operators of the form $P=P^{\prime}+P^{\prime \prime}+R$ with $P^{\prime}, R$ as above and $P^{\prime \prime} \in \Psi_{\mathrm{b}}^{m}$, as in part (2) of [30, Theorem 5.1], where the tame estimate now also involves the $C^{N}$-norm of the 'smooth part' $P$ " of the operator for some (s-dependent) $N$. Since in our application $P^{\prime \prime}$ will only depend on finitely many complex parameters, there is no need to prove an estimate which is also tame with respect to the $C^{N}$-norm of $P^{\prime \prime}$; however, this could easily be done in principle.

4.2. Real principal type propagation; radial points. Tame estimates for real principal type propagation and propagation near radial points can be deduced from a careful analysis of the proofs of the corresponding results in [30]. The main observation is that the regularity requirements, given in the footnotes to the proofs of these results in [30], indicate what regularity is needed to estimate the corresponding terms: For example, an operator in $A \in \Psi^{m ; 0} H_{\mathrm{b}}^{s}$ with $m \geq 0$ maps $H_{\mathrm{b}}^{m / 2}$ to $H_{\mathrm{b}}^{-m / 2}$ under the condition $s>n / 2+m / 2$, which is to say that one has a bound

$$
\|A u\|_{-m / 2} \lesssim\|A\|_{m-1, n / 2+m / 2+\epsilon}\|u\|_{m / 2}, \quad \epsilon>0 .
$$

This means that the only places where one needs to use tame operator bounds for operators with coefficients of regularity $s$ are those where the condition for mapping properties etc. to hold reads $s \gtrsim \sigma$ where $\sigma$ is the regularity of the target space, i.e. where $\sigma$ is comparable to the regularity $s$ of the coefficients.

We again only prove the tame real principal type estimate in the interior; the estimate near the boundary is proved in the same way, see also the discussion at the end of $\S 4.1$.

Proposition 4.2. (Cf. [30, Theorem 6.6].) Let $m, r, s, \widetilde{s} \in \mathbb{R}$. Suppose $P_{m} \in$ $H_{\mathrm{b}}^{s} \Psi_{\mathrm{b}}^{m}\left(\overline{\mathbb{R}_{+}^{n}}\right)$ has a real, scalar, homogeneous principal symbol $p_{m}$, and let $P_{m-1} \in$ $H_{\mathrm{b}}^{s-1} \Psi_{\mathrm{b}}^{m-1}\left(\overline{\mathbb{R}_{+}^{n}}\right), R \in \Psi_{\mathrm{b}}^{m-2 ; 0} H_{\mathrm{b}}^{s-1}\left(\overline{\mathbb{R}_{+}^{n}}\right)$. Let $P=P_{m}+P_{m-1}+R$. Suppose $s$ and $\widetilde{s}$ are such that

$$
\widetilde{s} \leq s-1, \quad s>n / 2+7 / 2+(2-\widetilde{s})_{+} .
$$

Let $\gamma:[0, T] \rightarrow{ }^{\mathrm{b}} S^{*} \overline{\mathbb{R}_{+}^{n}}$ be a segment of a null-bicharacteristic of $p_{m}$ with $\gamma(0)=\zeta_{0}$. Then for all $A \in \Psi_{\mathrm{b}}^{0}$ elliptic at $\zeta_{0}$, there exist $B \in \Psi_{\mathrm{b}}^{0}$ elliptic at $\gamma(T)$ and $G \in \Psi_{\mathrm{b}}^{0}$ elliptic on $\gamma([0, T])$ such that the following holds: If $u \in H_{\mathrm{b}}^{\widetilde{s}+m-3 / 2, r}\left(\overline{\mathbb{R}_{+}^{n}}\right)$ satisfies $P u=f \in H_{\mathrm{b}}^{\widetilde{s}, r}\left(\overline{\mathbb{R}_{+}^{n}}\right)$, and $\zeta_{0} \notin \mathrm{WF}_{\mathrm{b}}^{\widetilde{s}+m-1, r}(u),{ }^{6}$ then $\gamma(t) \notin \mathrm{WF}_{\mathrm{b}}^{\widetilde{s}+m-1, r}(u)$ for all

\footnotetext{
${ }^{6}$ Recall that for $u \in H_{\mathrm{b}}^{-\infty, r}$, we say $\zeta_{0} \notin \mathrm{WF}_{\mathrm{b}}^{\sigma, r}(u)$ if and only if there exists $A \in \Psi_{\mathrm{b}}^{0}$, elliptic at $\zeta_{0}$, such that $A u \in H_{\mathrm{b}}^{\sigma, r}$.
} 
$t \in[0, T] ;$ quantitatively, for $\widetilde{s} \leq n / 2+1, \epsilon>0$,

$$
\begin{aligned}
& \|B u\|_{\widetilde{s}+m-1, r}
\end{aligned}
$$

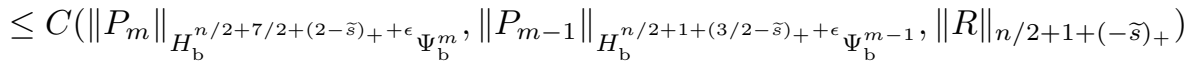

$$
\begin{aligned}
& \times\left(\|u\|_{\widetilde{s}+m-3 / 2, r}+\|A u\|_{\widetilde{s}+m-1, r}+\|G f\|_{\widetilde{s}, r}\right) .
\end{aligned}
$$

Moreover, for $\widetilde{s}>n / 2+1, \epsilon>0$, there is a tame estimate

$$
\begin{aligned}
\|B u\|_{\widetilde{s}+m-1, r} \leq L( & \left\|P_{m}\right\|_{H_{\mathrm{b}}^{n / 2+7 / 2+\epsilon} \Psi_{\mathrm{b}}^{m}},\left\|P_{m-1}\right\|_{H_{\mathrm{b}}^{n / 2+1+\epsilon} \Psi_{\mathrm{b}}^{m-1}},\|R\|_{n / 2+\epsilon} ; \\
& \left\|P_{m}\right\|_{H_{\mathrm{b}}^{s} \Psi_{\mathrm{b}}^{m}},\left\|P_{m-1}\right\|_{H_{\mathrm{b}}^{s-1} \Psi_{\mathrm{b}}^{m-1}},\|R\|_{m-2, s-1} ; \\
& \left.\|u\|_{n / 2-1 / 2+m+\epsilon} ;\|u\|_{\widetilde{s}+m-3 / 2, r},\|A u\|_{\widetilde{s}+m-1, r},\|G f\|_{\widetilde{s}, r}\right) .
\end{aligned}
$$

A simpler, but less precise, statement of Proposition 4.2, ignoring $P_{m-1}$ and $R$ for brevity, is the following: For sufficiently large $d>0$, and for $s, \widetilde{s}$ with $s \geq \widetilde{s}+d$, $\widetilde{s} \geq d$, one has an estimate

$$
\begin{aligned}
\|B u\|_{\tilde{s}+m-1} \leq C\left(\left\|P_{m}\right\|_{H_{\mathrm{b}}^{d} \Psi_{\mathrm{b}}^{m}}\right)\left(\|u\|_{\tilde{s}+m-3 / 2}+\|f\|_{\tilde{s}}\right. & \\
& \left.+\left\|P_{m}\right\|_{H_{\mathrm{b}}^{s} \Psi_{\mathrm{b}}^{m}}\left(\|A u\|_{d}+\|G f\|_{d}\right)\right),
\end{aligned}
$$

likewise with the weight $r$ added.

Proof of Proposition 4.2. We follow the proof of the regularity result in [30] and state the estimates needed to establish (4.6) and (4.7) along the way. Using the notation of the proof of [30, Theorem 6.6], but now calling the regularization parameter $\delta$, in particular $\check{A}_{\delta} \in \Psi_{\mathrm{b}}^{\widetilde{s}+(m-1) / 2}$ is the regularized commutant, which depends on a positive constant $M$ chosen below, and putting $\widetilde{f}=f-R u$, we have, assuming $m \geq 1$ and $\widetilde{s} \geq(5-m) / 2$ for now,

$$
\begin{aligned}
\operatorname{Re} & \left\langle i \check{A}_{\delta}^{*}\left[P_{m}, \check{A}_{\delta}\right] u, u\right\rangle \\
& =\frac{1}{2}\left\langle i\left(P_{m}-P_{m}^{*}\right) \check{A}_{\delta} u, \check{A}_{\delta} u\right\rangle-\operatorname{Re}\left\langle i \check{A}_{\delta} \tilde{f}, \check{A}_{\delta} u\right\rangle+\operatorname{Re}\left\langle i \check{A}_{\delta} P_{m-1} u, \check{A}_{\delta} u\right\rangle \\
& \equiv I+I I+I I I .
\end{aligned}
$$

For $\epsilon>0$, we can bound the first term by

$$
|I| \lesssim\left\|P_{m}\right\|_{H_{\mathrm{b}}^{n / 2+1+(m-1) / 2+\epsilon} \Psi_{\mathrm{b}}^{m}}\left\|\check{A}_{\delta} u\right\|_{(m-1) / 2}^{2}
$$

the second one by

$$
|I I| \lesssim\left\|\check{A}_{\delta} f\right\|_{-(m-1) / 2}^{2}+\|R u\|_{\widetilde{s}}^{2}+\left\|\check{A}_{\delta} u\right\|_{(m-1) / 2}^{2},
$$

where in turn

$$
\|R u\|_{\widetilde{s}} \lesssim \begin{cases}\|R\|_{m-2 ; n / 2+(-\widetilde{s})_{+}+t}\|u\|_{\widetilde{s}+m-2}, & \widetilde{s} \leq n / 2+t \\ \|R\|_{m-2 ; n / 2+\epsilon}\|u\|_{\tilde{s}+m-2}+\|R\|_{m-2 ; s-1}\|u\|_{n / 2+m-2+\epsilon}, & \widetilde{s} \geq 0\end{cases}
$$

for $t>0$ by Proposition 3.1. We estimate the third term by

$$
|I I I| \lesssim\left\|P_{m-1}\right\|_{H_{\mathrm{b}}^{\max (n / 2+\epsilon,(m-1) / 2)} \Psi_{\mathrm{b}}^{m-1}}\left\|\check{A}_{\delta} u\right\|_{(m-1) / 2}^{2}+\left|\left\langle\left[\check{A}_{\delta}, P_{m-1}\right] u, \check{A}_{\delta} u\right\rangle\right|
$$

and further, with $R_{2} \in \Psi_{\mathrm{b}}^{\widetilde{s}+(m-1) / 2-1} \circ \Psi^{m-1 ; 0} H_{\mathrm{b}}^{s-2}$ denoting a part of the expansion of $\left[\check{A}_{\delta}, P_{m-1}\right]$ as defined after [30, Footnote 28],

$$
\left|\left\langle\left[\check{A}_{\delta}, P_{m-1}\right] u, \check{A}_{\delta} u\right\rangle\right| \leq C(M)\left\|P_{m-1}\right\|_{H_{\mathrm{b}}^{n / 2+1+(m / 2-1)_{+}+\epsilon} \Psi_{\mathrm{b}}^{m-1}}\|u\|_{\widetilde{s}+m-3 / 2}^{2}
$$




$$
+\left\|R_{2} u\right\|_{-(m-1) / 2}^{2}+\left\|\check{A}_{\delta} u\right\|_{(m-1) / 2}^{2},
$$

where

$$
\begin{aligned}
& \left\|R_{2} u\right\|_{-(m-1) / 2} \\
& \leq C(M)\left\{\begin{array}{cl}
\left\|P_{m-1}\right\|_{H_{\mathrm{b}}^{n / 2+1+(1-\tilde{s})+}+\epsilon} \Psi_{\mathrm{b}}^{m-1}\|u\|_{\tilde{s}+m-2}, & \widetilde{s} \leq n / 2+1+\epsilon, \\
\left\|P_{m-1}\right\|_{H_{\mathrm{b}}^{n / 2+1+\epsilon} \Psi_{\mathrm{b}}^{m-1}\|u\|_{\tilde{s}+m-2}} & \\
+\left\|P_{m-1}\right\|_{H_{\mathrm{b}}^{s-1} \Psi_{\mathrm{b}}^{m-1}}\|u\|_{n / 2+m-1+\epsilon}, & \widetilde{s} \geq 1 .
\end{array}\right.
\end{aligned}
$$

Therefore, we obtain, see [30, Equation (6.24)],

$$
\begin{aligned}
\operatorname{Re}\left\langle\left(i \check{A}_{\delta}^{*}\left[P_{m}, \check{A}_{\delta}\right]+B_{\delta}^{*} B_{\delta}+M^{2}\left(\Lambda \check{A}_{\delta}\right)^{*}\left(\Lambda \check{A}_{\delta}\right)-E_{\delta}\right) u, u\right\rangle \\
\geq-\left|\left\langle E_{\delta} u, u\right\rangle\right|-\left\|\check{A}_{\delta} f\right\|_{-(m-1) / 2}^{2}-L^{2}+\left\|B_{\delta} u\right\|_{L_{\mathrm{b}}^{2}}^{2},
\end{aligned}
$$

where

$$
M=M\left(\left\|P_{m}\right\|_{H_{\mathrm{b}}^{n / 2+1+(m-1) / 2+\epsilon} \Psi_{\mathrm{b}}^{m}},\left\|P_{m-1}\right\|_{\left.H_{\mathrm{b}}^{\max (n / 2+\epsilon,(m-1) / 2)} \Psi_{\mathrm{b}}^{m-1}\right),}\right.
$$

and $L$ is 'tame'; more precisely, for $\widetilde{s} \leq n / 2+t, t>0$,

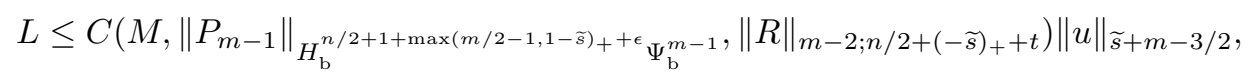

and for $\widetilde{s} \geq 1$,

$$
\begin{aligned}
& L=L\left(M,\left\|P_{m-1}\right\|_{H_{\mathrm{b}}^{n / 2+1+(m / 2-1)_{+}+\epsilon} \Psi_{\mathrm{b}}^{m-1}},\|R\|_{m-2, n / 2+\epsilon} ;\right. \\
& \left.\quad\left\|P_{m-1}\right\|_{H_{\mathrm{b}}^{s-1} \Psi_{\mathrm{b}}^{m-1}},\|R\|_{m-2 ; s-1} ;\|u\|_{n / 2+m-1+\epsilon} ;\|u\|_{\tilde{s}+m-3 / 2}\right) .
\end{aligned}
$$

Next, in order to exploit the positive commutator of the principal symbols of $P_{m}$ and $\check{A}_{\delta}$ in the estimate (4.8), we introduce operators $J^{ \pm} \in \Psi_{\mathrm{b}}^{ \pm(\widetilde{s}+(m-1) / 2-1)}$ with principal symbols $j^{ \pm}$such that $J^{+} J^{-}-I \in \Psi_{\mathrm{b}}^{-\infty}$; then

$$
i J^{-} \check{A}_{\delta}^{*}\left[P_{m}, \check{A}_{\delta}\right]=\mathrm{Op}\left(j^{-} \check{a}_{\delta} H_{p_{m}} \check{a}_{\delta}\right)+R_{1}+R_{2}+R_{3}+R_{4},
$$

see [30, Equation (6.27)], where

$$
\left|\left\langle R_{j} u,\left(J^{+}\right)^{*} u\right\rangle\right| \leq C(M)\left\|P_{m}\right\|_{H_{\mathrm{b}}^{n / 2+2+m / 2+\epsilon} \Psi_{\mathrm{b}}^{m}}\|u\|_{\widetilde{s}+m-3 / 2}^{2}, \quad j=1,3,4,
$$

and $R_{2} \in \Psi_{\mathrm{b}}^{\widetilde{s}+(m-1) / 2-1} \circ \Psi^{m ; 0} H_{\mathrm{b}}^{s-2}$, hence

$$
\begin{aligned}
& \left|\left\langle R_{2} u,\left(J^{+}\right)^{*} u\right\rangle\right| \\
& \leq C(M)\left\{\begin{array}{cl}
\left(1+\left\|P_{m}\right\|_{H_{\mathrm{b}}^{n / 2+2+(3 / 2-\widetilde{s})+}+\epsilon}^{2} \Psi_{\mathrm{b}}^{m}\right)\|u\|_{\widetilde{s}+m-3 / 2}^{2} & \forall \widetilde{s}, \\
\left(1+\left\|P_{m}\right\|_{H_{\mathrm{b}}^{n / 2+2+\epsilon} \Psi_{\mathrm{b}}^{m}}^{2}\right)\|u\|_{\widetilde{s}+m-3 / 2}^{2} & \\
+\left\|P_{m}\right\|_{H_{\mathrm{b}}^{s} \Psi_{\mathrm{b}}^{m}}^{2}\|u\|_{n / 2-1 / 2+m+\epsilon}^{2} & \widetilde{s} \geq 3 / 2 .
\end{array}\right.
\end{aligned}
$$

Thus, further following the proof in [30] to equation (6.28) and beyond, it remains to bound

$$
\operatorname{Re}\left\langle\operatorname{Op}\left(j^{-} f_{\delta} / j^{+}\right)\left(J^{+}\right)^{*} u,\left(J^{+}\right)^{*} u\right\rangle+\operatorname{Re}\left\langle R^{\prime} u,\left(J^{+}\right)^{*} u\right\rangle, \quad R^{\prime} \in \Psi^{\widetilde{s}+3(m-1) / 2 ; 0} H_{\mathrm{b}}^{s-1},
$$

from below, which is accomplished by

$$
\begin{gathered}
\left|\left\langle R^{\prime} u,\left(J^{+}\right)^{*} u\right\rangle\right| \leq C(M)\left\|P_{m}\right\|_{H_{\mathrm{b}}^{n / 2+1+m / 2+\epsilon} \Psi_{\mathrm{b}}^{m}}\|u\|_{\widetilde{s}+m-3 / 2}^{2}, \\
\operatorname{Re}\left\langle\operatorname{Op}\left(j^{-} f_{\delta} / j^{+}\right)\left(J^{+}\right)^{*} u,\left(J^{+}\right)^{*} u\right\rangle \geq-C(M)\left\|P_{m}\right\|_{H_{\mathrm{b}}^{n / 2+3+m / 2+\epsilon} \Psi_{\mathrm{b}}^{m}}\|u\|_{\widetilde{s}+m-3 / 2}^{2} .
\end{gathered}
$$


Lastly, for general $m \in \mathbb{R}$, we rewrite the equation $P u=f$ as $P \Lambda^{+}\left(\Lambda^{-} u\right)=f+P R u$ with $\Lambda^{ \pm} \in \Psi_{\mathrm{b}}^{\mp\left(m-m_{0}\right)}, R \in \Psi_{\mathrm{b}}^{-\infty}$, where $m_{0} \geq 1$; hence, replacing $P$ by $P \Lambda^{+}, u$ by $\Lambda^{-} u$ and $m$ by $m_{0}$ in the above estimates is equivalent to just replacing $m$ by $m_{0}$ in the b-Sobolev norms of the coefficients of $P$. Choosing $m_{0}=1+2(2-\widetilde{s})_{+}$as in [30] then implies the estimates (4.6) and (4.7) with $B=B_{0}, G$ an elliptic multiple of $\check{A}_{0}$, and $A$ elliptic on the microsupport of $E_{0}$.

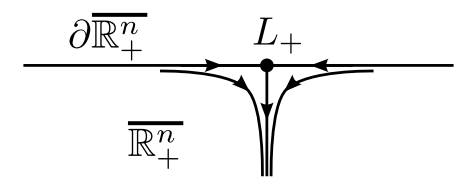

FiguRE 3. Null-bicharacteristic flow near a radial set, here a sink $L_{+} \subset{ }^{\mathrm{b}} S_{\partial \overline{\mathbb{R}}_{+}^{n}}^{*} \overline{\mathbb{R}_{+}^{n}}$ for the flow within the boundary ${ }^{\mathrm{b}} S_{\partial \mathbb{R}_{+}^{n}}^{*} \overline{\mathbb{R}_{+}^{n}}$, with an unstable direction transversal to the boundary. At a source $L_{-}$ within the boundary, with transversal stable direction, the direction of the flow would be reversed.

In a similar manner, we can analyze the proof of the radial point estimate, see Figure 3 for an illustration, obtaining, in the notation of [30, §6.4]:

Proposition 4.3. Let $m, r, s, \widetilde{s} \in \mathbb{R}, \alpha>0$. Let $P=P_{m}+P_{m-1}+R$, where $P_{j}=P_{j}^{\prime}+P_{j}^{\prime \prime}, j=m, m-1$, with $P_{m}^{\prime} \in H_{\mathrm{b}}^{s, \alpha} \Psi_{\mathrm{b}}^{m}\left(\overline{\mathbb{R}_{+}^{n}}\right)$ and $P_{m}^{\prime \prime} \in \Psi_{\mathrm{b}}^{m}\left(\overline{\mathbb{R}_{+}^{n}}\right)$ having real, scalar, homogeneous principal symbols $p_{m}^{\prime}$ and $p_{m}^{\prime \prime}$, respectively; moreover $P_{m-1}^{\prime} \in H_{\mathrm{b}}^{s-1, \alpha} \Psi_{\mathrm{b}}^{m-1}\left(\overline{\mathbb{R}_{+}^{n}}\right), P_{m-1}^{\prime \prime} \in \Psi_{\mathrm{b}}^{m-1}\left(\overline{\mathbb{R}_{+}^{n}}\right)$ and $R=R^{\prime}+R^{\prime \prime}$ with $R^{\prime} \in \Psi_{\mathrm{b}}^{m-2 ; 0} H_{\mathrm{b}}^{s-1, \alpha}\left(\overline{\mathbb{R}_{+}^{n}}\right)$ and $R^{\prime \prime} \in \Psi_{\mathrm{b}}^{m-2}\left(\overline{\mathbb{R}_{+}^{n}}\right)$. Suppose that the conditions (1)-(4) in $[30, \S 6.4]$ hold for $p=p_{m}^{\prime \prime}$, and

$$
\sigma_{\mathrm{b}, m-1}\left(\frac{1}{2 i}\left(\left(P_{m}^{\prime \prime}+P_{m-1}^{\prime \prime}\right)-\left(P_{m}^{\prime \prime}+P_{m-1}^{\prime \prime}\right)^{*}\right)\right)= \pm \widehat{\beta} \beta_{0} \rho^{m-1} \text { at } L_{ \pm},
$$

where $\widehat{\beta} \in \mathcal{C}^{\infty}\left(L_{ \pm}\right)$is self-adjoint at every point. Finally, assume that $s$ and $\widetilde{s}$ satisfy

$$
\widetilde{s} \leq s-1, \quad s>n / 2+7 / 2+(2-\widetilde{s})_{+} .
$$

Suppose $u \in H_{\mathrm{b}}^{\widetilde{s}+m-3 / 2, r}\left(\overline{\mathbb{R}_{+}^{n}}\right)$ is such that $P u=f \in H_{\mathrm{b}}^{\widetilde{s}, r}\left(\overline{\mathbb{R}_{+}^{n}}\right)$.

(1) If $\widetilde{s}+(m-1) / 2-1+\inf _{L_{ \pm}}(\widehat{\beta}-r \widetilde{\beta})>0$, let us assume that in a neighborhood of $L_{ \pm}, \mathcal{L}_{ \pm} \cap\{x>0\}$ is disjoint from $\mathrm{WF}_{\mathrm{b}}^{\widetilde{s}+m-1, r}(u)$.

(2) If $\widetilde{s}+(m-1) / 2+\sup _{L_{ \pm}}(\widehat{\beta}-r \widetilde{\beta})<0$, let us assume that a punctured neighborhood of $L_{ \pm}$, with $L_{ \pm}$removed, in $\Sigma \cap{ }^{\mathrm{b}} S_{\partial \mathbb{R}_{+}^{n}}^{*} \overline{\mathbb{R}_{+}^{n}}$ is disjoint from $\mathrm{WF}_{\mathrm{b}}^{\widetilde{s}+m-1, r}(u)$.

Then in both cases, $L_{ \pm}$is disjoint from $\mathrm{WF}_{\mathrm{b}}^{\widetilde{s}+m-1, r}(u)$.

Quantitatively, for every neighborhood $U$ of $L_{ \pm}$, there exist $B_{0}, B_{1} \in \Psi_{\mathrm{b}}^{0}$ elliptic at $L_{ \pm}, A \in \Psi_{\mathrm{b}}^{0}$ with microsupport in the respective a priori control region in the two cases above, with $\mathrm{WF}_{\mathrm{b}}^{\prime}(A), \mathrm{WF}_{\mathrm{b}}^{\prime}\left(B_{j}\right) \subset U, j=1,2$, and $\chi \in \mathcal{C}_{\mathrm{c}}^{\infty}(U)$, such for

\footnotetext{
${ }^{7}$ We recall that for an operator $A=\mathrm{Op}(a) \in \Psi_{\mathrm{b}}^{m}$, the operator wave front set $\mathrm{WF}_{\mathrm{b}}^{\prime}(A) \subset$ ${ }^{\mathrm{b}} S^{*} \overline{\mathbb{R}_{+}^{n}}$ is the complement of the largest open set in which the symbol $a$ is of order $-\infty$.
} 
$\widetilde{s} \leq n / 2+1, \epsilon>0$, we have, with implicit dependence of the appearing constants on seminorms of the smooth operators $P_{m}^{\prime \prime}, P_{m-1}^{\prime \prime}$ and $R^{\prime \prime}$ :

$$
\begin{gathered}
\left\|B_{0} u\right\|_{\widetilde{s}+m-1, r} \leq C\left(\left\|P_{m}^{\prime}\right\|_{H_{\mathrm{b}}^{n / 2+7 / 2+(2-\widetilde{s})_{+}+\epsilon, \alpha} \Psi_{\mathrm{b}}^{m}},\right. \\
\left.\left\|P_{m-1}^{\prime}\right\|_{H_{\mathrm{b}}^{n / 2+1+(3 / 2-\widetilde{s})_{+}+\epsilon, \alpha} \Psi_{\mathrm{b}}^{m-1}},\left\|R^{\prime}\right\|_{m-2, n / 2+1+(-\widetilde{s})_{+}}\right) \\
\times\left(\|u\|_{\tilde{s}+m-3 / 2, r}+\|A u\|_{\widetilde{s}+m-1, r}+\left\|B_{1} f\right\|_{\widetilde{s}, r}+\|\chi f\|_{\widetilde{s}-1, r}\right) .
\end{gathered}
$$

Moreover, for $\widetilde{s}>n / 2+1, \epsilon>0$, there is a tame estimate

$$
\begin{aligned}
& \left\|B_{0} u\right\|_{\widetilde{s}+m-1, r} \leq L\left(\left\|P_{m}^{\prime}\right\|_{H_{\mathrm{b}}^{n / 2+7 / 2+\epsilon, \alpha} \Psi_{\mathrm{b}}^{m}},\left\|P_{m-1}^{\prime}\right\|_{H_{\mathrm{b}}^{n / 2+1+\epsilon, \alpha} \Psi_{\mathrm{b}}^{m-1}},\left\|R^{\prime}\right\|_{m-2, n / 2+\epsilon}\right. \\
& \left.\left\|P_{m}^{\prime}\right\|_{H_{\mathrm{b}}^{s, \alpha} \Psi_{\mathrm{b}}^{m},},\left\|P_{m-1}^{\prime}\right\|_{H_{\mathrm{b}}^{s-1, \alpha} \Psi_{\mathrm{b}}^{m-1},\left\|R^{\prime}\right\|_{m-2, s-1} ;\|u\|_{n / 2-1 / 2+m+\epsilon},\|f\|_{n / 2-1+\epsilon}} ; \quad\|u\|_{\widetilde{s}+m-3 / 2, r},\|A u\|_{\widetilde{s}+m-1, r},\left\|B_{1} f\right\|_{\widetilde{s}, r},\|\chi f\|_{\widetilde{s}-1, r}\right) .
\end{aligned}
$$

Proof. One detail changes as compared to the previous proof: While it still suffices to only assume microlocal regularity $B_{2} f \in H_{\mathrm{b}}^{\widetilde{s}, r}$ at $L_{ \pm}$, we now in addition need to assume local regularity $\chi f \in H_{\mathrm{b}}^{\widetilde{s}-1, r}$, which is due to the use of elliptic regularity in the proof given in [30].

Remark 4.4. The above proposition provides tame estimates at (generalized) radial sets provided an open neighborhood of the radial set can be embedded as an open subset of $\overline{\mathbb{R}_{+}^{n}}$. While this holds for Kerr-de Sitter spaces, it does not hold in general. Thus, making the non-smooth positive commutator argument (which is not (micro)local within the radial set) work in general either requires analyzing the behavior of non-smooth operators under changes of coordinates and building a suitable invariant calculus; or, more easily, if we assume that $P=P_{m}+P_{m-1}+R$ with $P_{m-j} \in H_{\mathrm{b}}^{s-j, \alpha} \Psi_{\mathrm{b}}^{m-j}$ (as before), $j=0,1$, and $R \in H_{\mathrm{b}}^{s-1, \alpha} \Psi_{\mathrm{b}}^{m-2}$, thus making a manifestly invariant assumption on $R$ and thus on $P$, one can directly introduce localizers in the argument and estimate the resulting error terms, see Remark 4.6 for details.

4.3. Non-trapping estimates at normally hyperbolic trapping. We now extend the proof of non-trapping estimates on weighted b-Sobolev spaces at normally hyperbolically trapped sets given in [32, Theorem 3.2] to the non-smooth setting.

To set this up, let $P_{0} \in \Psi_{\mathrm{b}}^{m}\left(\overline{\mathbb{R}_{+}^{n}}\right)$ with

$$
\frac{1}{2 i}\left(P_{0}-P_{0}^{*}\right)=E_{1} \in \Psi_{\mathrm{b}}^{m-1}\left(\overline{\mathbb{R}_{+}^{n}}\right),
$$

where the adjoint is taken with respect to a fixed smooth b-density; an example to keep in mind here and in what follows is $P_{0}=\square_{g}$ for a smooth Lorentzian b-metric $g$ on $\overline{\mathbb{R}_{+}^{n}}$, considered a coordinate patch of Kerr-de Sitter space, in which case $E_{1}=0$, and the threshold weight in Theorem 4.5 below is $r=0$. Let $p_{0}$ be the principal symbol of $P_{0}$. Let us use the coordinates $(z ; \zeta)=(x, y ; \lambda, \eta)$ on ${ }^{\mathrm{b}} T^{*} \overline{\mathbb{R}_{+}^{n}}$ and write $M=\overline{\mathbb{R}_{+}^{n}}, X=\partial \overline{\mathbb{R}_{+}^{n}}$. With $\Sigma \subset{ }^{\mathrm{b}} S^{*} M$ denoting the characteristic set of $P_{0}$, we make the following assumptions:

(1) $\Gamma \subset \Sigma \cap{ }^{\mathrm{b}} S_{X}^{*} M$ is a smooth submanifold disjoint from the image of $T^{*} X \backslash o$, so $x D_{x}$ is elliptic near $\Gamma$,

(2) $\Gamma_{+}$is a smooth submanifold of $\Sigma \cap{ }^{\mathrm{b}} S_{X}^{*} M$ in a neighborhood $U_{1}$ of $\Gamma$,

(3) $\Gamma_{-}$is a smooth submanifold of $\Sigma$ transversal to $\Sigma \cap{ }^{\mathrm{b}} S_{X}^{*} M$ in $U_{1}$,

(4) $\Gamma_{+}$has codimension 2 in $\Sigma, \Gamma_{-}$has codimension 1 , 
(5) $\Gamma_{+}$and $\Gamma_{-}$intersect transversally in $\Sigma$ with $\Gamma_{+} \cap \Gamma_{-}=\Gamma$,

(6) the vector field $V$ is tangent to both $\Gamma_{+}$and $\Gamma_{-}$, and thus to $\Gamma$,

(7) $\Gamma_{+}$is backward trapped for the Hamilton flow, $\Gamma_{-}$is forward trapped; in particular, $\Gamma$ is a trapped set.

See Figure 4 for the setting.

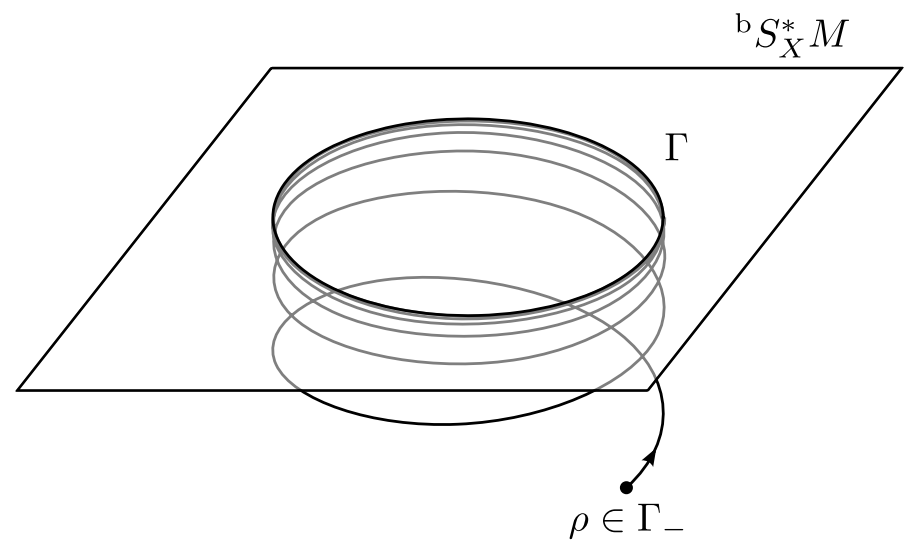

Figure 4. An exemplary situation with trapping: Shown are the (projection from ${ }^{\mathrm{b}} S^{*} M$ to the base $M$ of the) trapped set $\Gamma$, the bcosphere bundle over $X$ as well as a forward bicharacteristic starting at a point $\rho \in \Gamma_{-}$.

In view of condition (1), we can take

$$
\rho=\langle\lambda\rangle=\left(1+\lambda^{2}\right)^{1 / 2} \text { near } \Gamma,
$$

appropriately extended to ${ }^{\mathrm{b}} \bar{T}^{*} M$, as the inverse of a boundary defining function of fiber infinity ${ }^{\mathrm{b}} S^{*} M$ in ${ }^{\mathrm{b}} \bar{T}^{*} M{ }^{8}$ Then, let

$$
V=\rho^{-m+1} H_{p_{0}}
$$

be the rescaled Hamilton vector field of $p_{0}$. We make quantitative assumptions related to condition (7): Let $\phi_{+} \in \mathcal{C}^{\infty}\left({ }^{\mathrm{b}} S^{*} M\right)$ be a defining function of $\Gamma_{+}$in ${ }^{\mathrm{b}} S_{X}^{*} M$, and let $\phi_{-} \in \mathcal{C}^{\infty}\left({ }^{\mathrm{b}} S^{*} M\right)$ be a defining function of $\Gamma_{-}$. Thus, $\Gamma_{+}$is defined within ${ }^{\mathrm{b}} S^{*} M$ by $x=0, \phi_{+}=0$. Let

$$
\widehat{p}_{0}=\rho^{-m} p_{0} .
$$

We then assume that

(8) $\phi_{+}$and $\phi_{-}$satisfy

$$
V \phi_{+}=-c_{+}^{2} \phi_{+}+\mu_{+} x+\nu_{+} \widehat{p}_{0}, \quad V \phi_{-}=c_{-}^{2} \phi_{-}+\nu_{-} \widehat{p}_{0},
$$

with $c_{ \pm}>0$ smooth near $\Gamma$ and $\mu_{+}, \nu_{ \pm}$smooth near $\Gamma$. This is consistent with the (in)stability of $\Gamma_{-}\left(\Gamma_{+}\right)$,

\footnotetext{
${ }^{8}$ We are working locally here, but we remark that invariantly, $\lambda$ is the b-principal symbol (restricted to ${ }^{\mathrm{b}} T_{X}^{*} M \backslash o$ ) of the operator $x D_{x}, x$ a boundary defining function, which is independent of the choice of $x$.
} 
(9) $x$ satisfies

$$
V x=-c_{\partial} x, \quad c_{\partial}>0,
$$

which is consistent with the stability of $\Gamma_{-}$,

(10) near $\Gamma$,

$$
\rho^{-1} V \rho=c_{f} x
$$

for some smooth $c_{f}$, which holds in view of our choice of $\rho$.

Here we recall from [22, Lemma 5.1], see also [23, Lemma 2.4], that in the closely related semiclassical setting (see the discussion prior to Theorem 5.5) one can arrange for any $\epsilon>0$ that

$$
0<\nu_{\min }-\epsilon<c_{ \pm}^{2}<\nu_{\max }+\epsilon
$$

where $\nu_{\min }$ and $\nu_{\max }$ are the minimal and maximal normal expansion rates; see $[22$, Equations (5.1) and (5.2)] for the definition of the latter, with $\nu_{\min }$ also given in (4.27) below. ${ }^{9}$ In particular, if $M$ is replaced by $[0, \infty) \times X$, and if $P_{0}$ is dilation invariant, then the semiclassical and the b-settings are equivalent via the Mellin transform and a rescaling, see e.g. [51, §3.1]; since in our general case $c_{ \pm} \mid{ }_{\mathrm{b}} S_{X}^{*} M$ is what matters, we can replace $P_{0}$ by $N\left(P_{0}\right)$, and in particular (4.17) applies, with the expansion rate calculated using $\left.p_{0}\right|_{\mathrm{b}} T_{X}^{*} M \cdot{ }^{10}$

We now perturb $P_{0}$ by a non-smooth operator $\widetilde{P}$, that is, we consider the operator

$$
P=P_{0}+\widetilde{P}, \quad \widetilde{P}=\widetilde{P}_{m}+\widetilde{P}_{m-1}+\widetilde{R},
$$

where for some fixed $\alpha>0$, we have $\widetilde{P}_{m-j} \in H_{\mathrm{b}}^{s-j, \alpha} \Psi_{\mathrm{b}}^{m-j}, j=0,1$, and $\widetilde{R} \in$ $\Psi_{\mathrm{b}}^{m-2 ; 0} H_{\mathrm{b}}^{s-1, \alpha}$.

We then have the following tame non-trapping estimate at $\Gamma$ :

Theorem 4.5. Using the above notation and making the above assumptions, let $s, \widetilde{s} \in \mathbb{R}$ be such that

$$
\widetilde{s} \leq s-1, \quad s>n / 2+7 / 2+(2-\widetilde{s})_{+} .
$$

Then for $r<-\sup _{\Gamma} \rho^{-m+1} \sigma_{\mathrm{b}, m-1}\left(E_{1}\right) / c_{\partial}$ and for any neighborhood $U$ of $\Gamma$, there exist $B_{0} \in \Psi_{\mathrm{b}}^{0}(M)$ elliptic at $\Gamma$ and $B_{1}, B_{2} \in \Psi_{\mathrm{b}}^{0}(M)$ with $\operatorname{WF}_{\mathrm{b}}^{\prime}\left(B_{j}\right) \subset U$, $j=0,1,2, \mathrm{WF}_{\mathrm{b}}^{\prime}\left(B_{2}\right) \cap \Gamma_{+}=\emptyset$, and $\chi \in \mathcal{C}_{\mathrm{c}}^{\infty}(U)$, such that the following holds for $u \in H_{\mathrm{b}}^{\widetilde{s}+m-3 / 2, r}\left(\overline{\mathbb{R}_{+}^{n}}\right)$ solving the equation $P u=f \in H_{\mathrm{b}}^{\widetilde{s}, r}\left(\overline{\mathbb{R}_{+}^{n}}\right):$ For $\widetilde{s} \leq n / 2+1$, $\epsilon>0$

$$
\begin{aligned}
& \left\|B_{0} u\right\|_{\widetilde{s}_{+}+m-1, r} \leq C\left(\left\|\widetilde{P}_{m}\right\|_{H_{\mathrm{b}}^{n / 2+7 / 2+(2-\widetilde{s})+}+\epsilon, \alpha} \Psi_{\mathrm{b}}^{m},\right.
\end{aligned}
$$

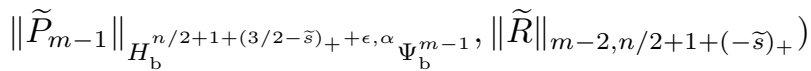

$$
\begin{aligned}
& \times\left(\|u\|_{\widetilde{s}+m-3 / 2, r}+\left\|B_{2} u\right\|_{\widetilde{s}+m-1, r}+\left\|B_{1} f\right\|_{\widetilde{s}, r}+\|\chi f\|_{\tilde{s}-1, r}\right) .
\end{aligned}
$$

Moreover, for $\widetilde{s}>n / 2+1, \epsilon>0$, there is a tame estimate

$$
\begin{aligned}
& \left\|B_{0} u\right\|_{\widetilde{s}+m-1, r} \leq L\left(\left\|\widetilde{P}_{m}\right\|_{H_{\mathrm{b}}^{n / 2+7 / 2+\epsilon, \alpha} \Psi_{\mathrm{b}}^{m}},\left\|\widetilde{P}_{m-1}\right\|_{H_{\mathrm{b}}^{n / 2+1+\epsilon, \alpha} \Psi_{\mathrm{b}}^{m-1}},\|\widetilde{R}\|_{m-2, n / 2+\epsilon} ;\right. \\
& \left\|\widetilde{P}_{m}\right\|_{H_{\mathrm{b}}^{s, \alpha} \Psi_{\mathrm{b}}^{m}},\left\|\widetilde{P}_{m-1}\right\|_{H_{\mathrm{b}}^{s-1, \alpha} \Psi_{\mathrm{b}}^{m-1}},\|\widetilde{R}\|_{m-2, s-1} ;\|u\|_{n / 2-1 / 2+m+\epsilon},\|f\|_{n / 2-1+\epsilon} ; \\
& \left.\|u\|_{\widetilde{s}+m-3 / 2, r},\left\|B_{2} u\right\|_{\tilde{s}+m-1, r},\left\|B_{1} f\right\|_{\widetilde{s}, r},\|\chi f\|_{\widetilde{s}-1, r}\right) .
\end{aligned}
$$

\footnotetext{
${ }^{9}$ The functions $c_{ \pm}$in $[22,23]$ are called $c_{ \pm}^{2}$ here.

${ }^{10}$ We rely on the setup described in [23] here, which, in contrast to [22], does not use a normal form for the operator $P_{0}$.
} 
On the other hand, for $r>-\inf _{\Gamma} \rho^{-m+1} \sigma_{\mathrm{b}, m-1}\left(E_{1}\right) / c_{\partial}$ and for appropriate $B_{2}$ with $\mathrm{WF}_{\mathrm{b}}^{\prime}\left(B_{2}\right) \cap \Gamma_{-}=\emptyset$, the estimates (4.20) and (4.21) hold as well. These estimates are understood in the sense that if all quantities on the right hand side are finite, then so is the left hand side, and the inequality holds.

We leave the statement of a less precise version of this Theorem in the spirit of the discussion following the statement of Proposition 4.2 to the reader.

Proof of Theorem 4.5. The main part of the argument, in particular the choice of the commutant, is a slight modification of the positive commutator argument of [32, Theorem 3.2]; the handling of the non-smooth terms is a modification of the proof of the radial point estimate, [30, Theorem 6.10]. In particular, the positivity comes from differentiating the weight $x^{-r}$ in the commutant. To avoid working in weighted b-Sobolev spaces for the non-smooth problem, we will conjugate $P$ by $x^{-r}$, giving an advantageous (here meaning negative) contribution to the imaginary part of the subprincipal symbol of the conjugated operator near $\Gamma$.

Throughout this proof, we denote operators and their symbols by the corresponding capital and lower case letters, respectively.

Concretely, put $\sigma=\widetilde{s}+m-1$, and define

$$
\begin{gathered}
u_{r}:=x^{-r} u \in H_{\mathrm{b}}^{\sigma-1 / 2}, \quad f_{r}:=x^{-r} f \in H_{\mathrm{b}}^{\sigma-m+1}, \\
P_{r}:=x^{-r} P x^{r}=P_{0, r}+\widetilde{P}_{r}, \quad P_{0, r}=x^{-r} P_{0} x^{r}, \widetilde{P}_{r}=x^{-r} \widetilde{P} x^{r},
\end{gathered}
$$

where

$$
\widetilde{P}_{r}=\widetilde{P}_{m, r}+\widetilde{P}_{m-1, r}+\widetilde{R}_{r}, \quad \widetilde{P}_{m-j, r} \in H_{\mathrm{b}}^{s-j, \alpha} \Psi_{\mathrm{b}}^{m-j}, \widetilde{R}_{r} \in \Psi_{\mathrm{b}}^{m-2 ; 0} H_{\mathrm{b}}^{s-1, \alpha} ;
$$

then $P_{r} u_{r}=f_{r}$, and we must show a non-trapping estimate for $u_{r}$ on unweighted b-Sobolev spaces. A simple computation shows that

$$
\frac{1}{2 i}\left(P_{0, r}-P_{0, r}^{*}\right)-\left(\frac{1}{2 i}\left(P_{0}-P_{0}^{*}\right)-\mathrm{Op}\left(r x^{-1} H_{p_{0}} x\right)\right) \in \Psi_{\mathrm{b}}^{m-2} ;
$$

but $x^{-1} H_{p_{0}} x=-\rho^{m-1} c_{\partial}$ with $c_{\partial}>0$ near $\Gamma$ by (4.15), hence, using (4.13),

$$
\frac{1}{2 i}\left(P_{0, r}-P_{0, r}^{*}\right)=E_{1}+E_{1}^{\prime}+B
$$

with $B, E_{1}^{\prime} \in \Psi_{\mathrm{b}}^{m-1}$, where $B$ has principal symbol $b=r c_{\partial} \rho^{m-1}$ near $\Gamma$, and $\mathrm{WF}_{\mathrm{b}}^{\prime}\left(E_{1}^{\prime}\right) \cap \Gamma=\emptyset$. Notice that by assumption on $r, B+E_{1}$ is elliptic on $\Gamma$.

We now turn to the positive commutator argument: Fix $0<\beta<\min (1, \alpha)$ and define

$$
\rho_{+}=\phi_{+}^{2}+x^{\beta} .
$$

Let $\chi_{0}(t)=e^{-1 / t}$ for $t>0$ and $\chi_{0}(t)=0$ for $t<0$, further $\chi \in \mathcal{C}_{\mathrm{c}}^{\infty}([0, R))$ for $R>0$ to be chosen below, $\chi \equiv 1$ near $0, \chi^{\prime} \leq 0$, and finally $\psi \in \mathcal{C}_{\mathrm{c}}^{\infty}((-R, R))$, $\psi \equiv 1$ near 0 . Define for $\kappa>0$, specified later,

$$
a=\rho^{\sigma-(m-1) / 2} \chi_{0}\left(\rho_{+}-\phi_{-}^{2}+\kappa\right) \chi\left(\rho_{+}\right) \psi\left(\widehat{p}_{0}\right) .
$$

On supp $a$, we have $\rho_{+} \leq R$, thus the argument of $\chi_{0}$ is bounded above by $R+$ $\kappa$. Moreover, $\phi_{-}^{2} \leq R+\kappa$ and $x \leq R^{1 / \beta}$, therefore $a$ is supported in any given neighborhood of $\Gamma$ if one chooses $R$ and $\kappa$ small. Notice that $a$ is merely a conormal symbol which does not grow at the boundary. However, b-analysis for operators with conormal coefficients can easily be seen to work without much additional work, in fact, a logarithmic change of variables essentially reduces such a b-analysis on $\overline{\mathbb{R}_{+}^{n}}$ 
to the analysis of operators corresponding to uniform symbols on $\mathbb{R}^{n}$. Moreover, the proofs of composition results of smooth and non-smooth b-ps.d.o.s presented in [30] go through without changes if one uses b-ps.d.o.s with non-growing conormal, instead of smooth, symbols. ${ }^{11}$

Define the regularizer $\varphi_{\delta}(\zeta)=(1+\delta \rho)^{-1}$ near $\Gamma$, and put $a_{\delta}=\varphi_{\delta} a$. Put $\widetilde{V}=\rho^{-m+1} H_{\widetilde{p}_{m, r}}$ and define $\widetilde{c}_{\partial}, \widetilde{c}_{f} \in H_{\mathrm{b}}^{s-1, \alpha}$ near $\Gamma$ by $\widetilde{V} x=-\widetilde{c}_{\partial} x, \rho^{-1} \widetilde{V} \rho=\widetilde{c}_{f} x$. Then, with $p_{m, r}=p_{0, r}+\widetilde{p}_{m, r}$, we obtain, using (4.14)-(4.16):

$$
\begin{gathered}
a_{\delta} H_{p_{m, r}} a_{\delta}=\varphi_{\delta}^{2} \rho^{2 \sigma} \chi_{0}^{2} \chi^{2} \psi^{2}\left(\sigma-(m-1) / 2-\delta \rho \varphi_{\delta}\right)\left(c_{f}+\widetilde{c}_{f}\right) x \\
-\varphi_{\delta}^{2} \rho^{2 \sigma} \chi_{0} \chi_{0}^{\prime} \chi^{2} \psi^{2}\left(2 c_{+}^{2} \phi_{+}^{2}+\beta c_{\partial} x^{\beta}-2 \mu_{+} \phi_{+} x-2 \nu_{+} \phi_{+} \widehat{p}_{0}\right. \\
\left.+2 c_{-}^{2} \phi_{-}^{2}+2 \nu_{-} \phi_{-} \widehat{p}_{0}-\widetilde{V} \phi_{+}^{2}+\beta \widetilde{c}_{\partial} x^{\beta}+\widetilde{V} \phi_{-}^{2}\right) \\
+\varphi_{\delta}^{2} \rho^{2 \sigma} \chi_{0}^{2} \chi \chi^{\prime} \psi^{2}\left(V \rho_{+}+\widetilde{V} \rho_{+}\right)+\varphi_{\delta}^{2} \rho^{2 \sigma} \chi_{0}^{2} \chi^{2} \psi \psi^{\prime}\left(V \widehat{p}_{0}+\widetilde{V} \widehat{p}_{0}\right) \\
=-c_{+}^{2} a_{+, \delta}^{2}-c_{-}^{2} a_{-, \delta}^{2}+a_{+, \delta} h_{+, \delta} p_{m, r}+a_{-, \delta} h_{-, \delta} p_{m, r}+e_{\delta}+g_{\delta}-f_{\delta},
\end{gathered}
$$

where, writing $\widehat{p}_{0}=\rho^{-m} p_{m, r}-\rho^{-m} \widetilde{p}_{m, r}$ in the second and third line,

$$
\begin{aligned}
& a_{ \pm, \delta}=\varphi_{\delta} \rho^{\sigma} \sqrt{2 \chi_{0} \chi_{0}^{\prime}} \chi \psi \phi_{ \pm} \\
& h_{ \pm, \delta}= \pm \varphi_{\delta} \rho^{\sigma-m} \sqrt{2 \chi_{0} \chi_{0}^{\prime}} \chi \psi \nu_{ \pm} \\
& e_{\delta}=\varphi_{\delta}^{2} \rho^{2 \sigma} \chi_{0}^{2} \chi \chi^{\prime} \psi^{2}\left(V \rho_{+}+\widetilde{V} \rho_{+}\right), \\
& g_{\delta}=\varphi_{\delta}^{2} \rho^{2 \sigma} \chi_{0}^{2} \chi^{2} \psi \psi^{\prime}\left(V \widehat{p}_{0}+\widetilde{V} \widehat{p}_{0}\right), \\
& f_{\delta}=\varphi_{\delta}^{2} \rho^{2 \sigma} \chi_{0} \chi^{2} \psi^{2}\left[\left(\beta\left(c_{\partial}+\widetilde{c}_{\partial}\right) x^{\beta}-2 \mu_{+} \phi_{+} x-\widetilde{V} \phi_{+}^{2}+\widetilde{V} \phi_{-}^{2}\right.\right. \\
&\left.\quad+2\left(\nu_{+} \phi_{+}-\nu_{-} \phi_{-}\right) \rho^{-m} \widetilde{p}_{m, r}\right) \chi_{0}^{\prime} \\
&\left.\quad-\left(\sigma-(m-1) / 2-\delta \rho \varphi_{\delta}\right)\left(c_{f}+\widetilde{c}_{f}\right) x \chi_{0}\right]
\end{aligned}
$$

Note that in the definition of $f_{\delta}$, by the choice of $\beta$ and using the fact that $\chi_{0}$ is bounded by a constant multiple of $\chi_{0}^{\prime}$ on its support, the constant being uniform for $R+\kappa<1$, the term $c_{\partial} x^{\beta}$ dominates all other terms on the support of $f_{\delta} \in$ $S^{2 \sigma ; \infty} H_{\mathrm{b}}^{s-1}$ for $R$ and $\kappa$ small enough, hence $f_{\delta} \geq 0$, and its contribution will be controlled by virtue of the sharp Gårding inequality. The term arising from $e_{\delta}$ will be controlled using the a priori regularity assumption of $u_{r}$ on $\Gamma_{-}$, and $g_{\delta}$, which is supported away from the characteristic set, will be controlled using elliptic regularity.

Proceeding with the argument, we first make the simplification $\widetilde{R}_{r}=0$ by replacing $f$ by $f-\widetilde{R}_{r} u_{r}$, and we assume $m \geq 1$ and $\widetilde{s} \geq(5-m) / 2$ for now. Then we

${ }^{11} \mathrm{~A}$ somewhat more direct way of dealing with this issue goes as follows: Assume, as one may, that $\ell:=\beta^{-1} \in \mathbb{N}$. Then even though $a$ is not a smooth symbol of $\overline{\mathbb{R}_{+}^{n}}$ with the standard smooth structure, it becomes smooth if one changes the smooth structure of $\frac{+}{\mathbb{R}_{+}^{n}}$ by blowing up the boundary to the $\ell$-th order, i.e. by taking $x^{\prime}=x^{\beta}$ as a boundary defining function, thus obtaining a manifold $M_{\ell}$, which is $\overline{\mathbb{R}_{+}^{n}}$ as a topological manifold, but with a different smooth structure; in particular, the function $x=\left(x^{\prime}\right)^{\ell}$ is smooth on $M_{\ell}$ in view of $\ell \in \mathbb{N}$. Moreover, the blow-down map $M_{\ell} \rightarrow \overline{\mathbb{R}_{+}^{n}}$ induces isomorphisms (see e.g. [40, §4.18])

$$
H_{\mathrm{b}}^{s^{\prime}, \gamma}\left(\overline{\mathbb{R}_{+}^{n}}\right) \cong H_{\mathrm{b}}^{s^{\prime}, \ell \gamma}\left(M_{\ell}\right), \quad s^{\prime}, \gamma \in \mathbb{R} .
$$

Therefore, one can continue to work on $\overline{\mathbb{R}_{+}^{n}}$, tacitly assuming that all functions and operators live on, and all computations are carried out on, $M_{\ell}$. 
have, as in the proof of [30, Theorem 6.10],

$$
\begin{aligned}
& \operatorname{Re}\left\langle i A_{\delta}^{*}\left[P_{0, r}+\widetilde{P}_{m, r}, A_{\delta}\right] u_{r}, u_{r}\right\rangle+\left\langle\frac{1}{2 i}\left(P_{0, r}-P_{0, r}^{*}\right) A_{\delta} u_{r}, A_{\delta} u_{r}\right\rangle \\
&=-\left\langle\frac{1}{2 i}\left(\widetilde{P}_{m, r}-\widetilde{P}_{m, r}^{*}\right) A_{\delta} u_{r}, A_{\delta} u_{r}\right\rangle \\
&-\operatorname{Re}\left\langle i A_{\delta} f, A_{\delta} u_{r}\right\rangle+\operatorname{Re}\left\langle i A_{\delta} \widetilde{P}_{m-1, r} u_{r}, A_{\delta} u_{r}\right\rangle
\end{aligned}
$$

Estimating each term on the right hand side as in the proof of [30, Theorem 6.10] and using (4.22), we obtain for any $\mu>0$ :

$$
\operatorname{Re}\left\langle\left(A_{\delta}^{*}\left(i\left[P_{0, r}+\widetilde{P}_{m, r}, A_{\delta}\right]+E_{1}+E_{1}^{\prime}+B\right) A_{\delta}\right) u_{r}, u_{r}\right\rangle \geq-C_{\mu}-\mu\left\|A_{\delta} u_{r}\right\|_{(m-1) / 2}^{2}
$$

Here and in what follows, we in particular absorb all terms involving $\left\|u_{r}\right\|_{\sigma-1 / 2}$ into the constant $C_{\mu}$. On the left hand side, the $E_{1}^{\prime}$-term can be dropped because of $\mathrm{WF}_{\mathrm{b}}^{\prime}\left(E_{1}^{\prime}\right) \cap \mathrm{WF}_{\mathrm{b}}^{\prime}(A)=\emptyset$ for sufficiently localized $a$. Moreover, the principal symbol of $E_{1}+B$ near $\Gamma$ is $e_{1}+b=-q^{2}$ with $q$ an elliptic symbol of order $(m-1) / 2$, since, by assumption on $r$, we have $e_{1}+r c_{\partial} \rho^{m-1}<0$ near $\Gamma$. Therefore, we can write $E_{1}+B=-Q^{*} Q+E_{1}^{\prime \prime}+E_{2}$, where $E_{1}^{\prime \prime} \in \Psi_{\mathrm{b}}^{m-1}, E_{2} \in \Psi_{\mathrm{b}}^{m-2}, \mathrm{WF}_{\mathrm{b}}^{\prime}\left(E_{1}^{\prime \prime}\right) \cap \Gamma=\emptyset$. Again, the resulting term in the pairing (4.24) involving $E_{1}^{\prime \prime}$ can be dropped; also, the term involving $E_{2}$ can be dropped at the cost of changing the constant $C_{\mu}$, since $u_{r} \in H_{\mathrm{b}}^{\sigma-1 / 2}$.

Hence, introducing $J^{ \pm} \in \Psi_{\mathrm{b}}^{ \pm(\sigma-(m-1) / 2-1)}$, with real principal symbols, satisfying $I-J^{+} J^{-} \in \Psi_{\mathrm{b}}^{-\infty}$, we get

$$
\operatorname{Re}\left\langle\operatorname{Op}\left(j^{-} a_{\delta} H_{p_{m, r}} a_{\delta}\right) u_{r},\left(J^{+}\right)^{*} u_{r}\right\rangle-\left\|Q A_{\delta} u_{r}\right\|_{0}^{2} \geq-C_{\mu}-\mu\left\|A_{\delta} u_{r}\right\|_{(m-1) / 2}^{2} .
$$

We now plug the commutator relation (4.23) into this estimate. We obtain several terms, which we bound as follows: First, since $j^{-} e_{\delta} \in\left(\mathcal{C}^{\infty}+H_{\mathrm{b}}^{s-1, \alpha}\right) S^{\sigma+(m-1) / 2+1}$ uniformly, $\mathrm{Op}\left(j^{-} e_{\delta}\right)$ is a uniformly bounded family of maps $H_{\mathrm{b}}^{\sigma} \rightarrow H_{\mathrm{b}}^{-(m+1) / 2}$; thus, choosing $\widetilde{E} \in \Psi_{\mathrm{b}}^{0}$ with $\mathrm{WF}_{\mathrm{b}}^{\prime}(\widetilde{E}) \subset U$ and with $\mathrm{WF}_{\mathrm{b}}^{\prime}(I-\widetilde{E})$ disjoint from supp $e_{\delta}$, we conclude

$$
\left|\left\langle\mathrm{Op}\left(j^{-} e_{\delta}\right) u_{r},\left(J^{+}\right)^{*} u_{r}\right\rangle\right| \leq C+\left|\left\langle\mathrm{Op}\left(j^{-} e_{\delta}\right) u_{r},\left(J^{+}\right)^{*} \widetilde{E} u_{r}\right\rangle\right| \leq C+\left\|B_{2} u_{r}\right\|_{\sigma}^{2}
$$

for some $B_{2} \in \Psi_{\mathrm{b}}^{0}$ with $\mathrm{WF}_{\mathrm{b}}^{\prime}\left(B_{2}\right) \cap \Gamma_{+}=\emptyset$.

Next, the term $\left\langle\mathrm{Op}\left(j^{-} g_{\delta}\right) u_{r},\left(J^{+}\right)^{*} u_{r}\right\rangle$ is uniformly bounded, as detailed in the proof of [30, Theorem 6.10]. Moreover, by the sharp Gårding inequality, see the argument in the proof of [30, Theorem 6.6],

$$
\operatorname{Re}\left\langle\mathrm{Op}\left(-j^{-} f_{\delta}\right) u_{r},\left(J^{+}\right)^{*} u_{r}\right\rangle \leq C .
$$

Further, we obtain two terms involving $h_{ \pm, \delta}$; introducing $B_{3} \in \Psi_{\mathrm{b}}^{0}$ elliptic on $\mathrm{WF}_{\mathrm{b}}^{\prime}(A)$, these can be bounded for $\mu>0$ by

$$
\begin{aligned}
& \left|\left\langle\mathrm{Op}\left(j^{-} a_{ \pm, \delta} h_{ \pm, \delta} p_{m, r}\right) u_{r},\left(J^{+}\right)^{*} u_{r}\right\rangle\right| \\
& \quad \leq C+\left|\left\langle\mathrm{Op}\left(j^{-} a_{ \pm, \delta} h_{ \pm, \delta}\right)\left(P_{0, r}+\widetilde{P}_{m, r}\right) u_{r},\left(J^{+}\right)^{*} u_{r}\right\rangle\right| \\
& \quad \leq C+\left|\left\langle H_{ \pm, \delta} f_{r}, A_{ \pm, \delta} u_{r}\right\rangle\right|+\left|\left\langle\mathrm{Op}\left(j^{-} a_{ \pm, \delta} h_{ \pm, \delta}\right) \widetilde{P}_{m-1, r} u_{r},\left(J^{+}\right)^{*} u_{r}\right\rangle\right| \\
& \quad \leq C+\mu\left\|A_{ \pm, \delta} u_{r}\right\|_{0}^{2}+C_{\mu}\left\|B_{3} f_{r}\right\|_{\sigma-m}^{2} .
\end{aligned}
$$


Here, for the first estimate, we employ [30, Theorem 3.12 (3)] to obtain

$$
\begin{aligned}
\mathrm{Op}\left(j^{-} a_{ \pm, \delta} h_{ \pm, \delta}\right) & \widetilde{P}_{m, r}-\mathrm{Op}\left(j^{-} a_{ \pm, \delta} h_{ \pm, \delta} \widetilde{p}_{m, r}\right) \\
=: & \Upsilon_{\delta} \in \Psi_{\mathrm{b}}^{\sigma+(m-1) / 2 ; 0} H_{\mathrm{b}}^{s-1}+\Psi_{\mathrm{b}}^{\sigma-(m-1) / 2-1} \circ \Psi^{m ; 0} H_{\mathrm{b}}^{s-1},
\end{aligned}
$$

and $\Upsilon_{\delta}$ is easily seen to be uniformly bounded from $H_{\mathrm{b}}^{\sigma-1 / 2}$ to $H_{\mathrm{b}}^{-m / 2}$, whereas $\left(J^{+}\right)^{*} u_{r} \in H_{\mathrm{b}}^{m / 2}$, thus $\left|\left\langle\Upsilon_{\delta} u_{r},\left(J^{+}\right)^{*} u_{r}\right\rangle\right| \leq C$. For the second estimate, we simply use $\left(P_{0, r}+\widetilde{P}_{m, r}\right) u_{r}=f_{r}-\widetilde{P}_{m-1, r} u_{r}$, and for the third estimate, we apply the Peter-Paul inequality to the first pairing; to bound the second pairing, we use the boundedness of $\widetilde{P}_{m-1, r}: H_{\mathrm{b}}^{\sigma-1 / 2} \rightarrow H_{\mathrm{b}}^{\sigma-m+1 / 2}$.

Finally, including the terms $c_{ \pm}^{2} a_{ \pm, \delta}^{2}$ into the estimate obtained from (4.25) by making use of the above estimates, we obtain

$$
\begin{aligned}
\left\|C_{+} A_{+, \delta} u_{r}\right\|_{0}^{2} & +\left\|C_{-} A_{-, \delta} u_{r}\right\|_{0}^{2}+\left\|Q A_{\delta} u_{r}\right\|_{0}^{2} \\
\leq C_{\mu}+\mu \| & A_{+, \delta} u_{r}\left\|_{0}^{2}+\mu\right\| A_{-, \delta} u_{r}\left\|_{0}^{2}+\mu\right\| A_{\delta} u_{r} \|_{(m-1) / 2}^{2} \\
& +\left\|B_{2} u_{r}\right\|_{\sigma}^{2}+\left\|B_{1} f_{r}\right\|_{\sigma-m+1}^{2}+C_{\mu}\left\|\chi f_{r}\right\|_{\sigma-m}^{2},
\end{aligned}
$$

where $B_{1} \in \Psi_{\mathrm{b}}^{0}$ is elliptic on $\mathrm{WF}_{\mathrm{b}}^{\prime}(A)$ with $\mathrm{WF}_{\mathrm{b}}^{\prime}\left(B_{1}\right) \subset U$, and $\chi \in \mathcal{C}_{\mathrm{c}}^{\infty}(M)$ is identically 1 near the projection of $\Gamma \subset{ }^{\mathrm{b}} S^{*} M$ to the base $M$. Since $c_{+}$and $c_{-}$ have positive lower bounds near $\Gamma$, we can absorb the terms on the right involving $A_{ \pm, \delta}$ into the left hand side by choosing $\mu$ sufficiently small, at the cost of changing the constant $C_{\mu}$; likewise, $\rho^{-(m-1) / 2} q$ has a positive lower bound near supp $a$, hence the term on the right involving $A_{\delta}$ can be absorbed into the left hand side for small $\mu$. Dropping the first two terms on the left hand side, we obtain the $H_{\mathrm{b}}^{\sigma}$-regularity of $u_{r}$ at $\Gamma$, hence $\mathrm{WF}_{\mathrm{b}}^{\sigma, r}(u) \cap \Gamma=\emptyset$, and a corresponding tame estimate, which follows from a careful analysis of the above argument as in the proof of Proposition 4.2.

Next, we remove the restriction $m \geq 1$ : Let $m_{0} \geq 1$. The idea, as before, is to rewrite $P u=f$ as $P \Lambda^{+}\left(\Lambda^{-} u\right)=f+P R u$, where $\Lambda^{ \pm} \in \Psi_{\mathrm{b}}^{ \pm\left(m_{0}-m\right)}$, with real principal symbols, satisfy $\Lambda^{+} \Lambda^{-}=I+R$. We now have to be a bit careful though to not change the imaginary part of the subprincipal symbol of $P \Lambda^{+}$at $\Gamma$. Concretely, we choose $\Lambda^{+}$self-adjoint with principal symbol $\lambda^{+}=\rho^{m_{0}-m}$ near $\Gamma$; then

$$
P_{0} \Lambda^{+}-\left(P_{0} \Lambda^{+}\right)^{*}=\Lambda^{+}\left(P_{0}-P_{0}^{*}\right)+\left[P_{0}, \Lambda^{+}\right] .
$$

Clearly, $\Lambda^{+}\left(P_{0}-P_{0}^{*}\right) \in x \Psi_{\mathrm{b}}^{m_{0}-1}+\Psi_{\mathrm{b}}^{m_{0}-2}$, and the principal symbol of the second term is

$$
\sigma_{\mathrm{b}, m_{0}-1}\left(\left[P_{0}, \Lambda^{+}\right]\right)=-i H_{p_{0}} \lambda^{+}=-i x\left(m_{0}-m\right) \rho^{m_{0}-1} c_{f}
$$

near $\Gamma$ by (4.16), hence, using (4.13),

$$
P_{0} \Lambda^{+}-\left(P_{0} \Lambda^{+}\right)^{*}=\Lambda^{+} E_{1}+x E_{1}^{\prime}+E_{1}^{\prime \prime}+E_{2}
$$

with $E_{1}^{\prime}, E_{1}^{\prime \prime} \in \Psi_{\mathrm{b}}^{m_{0}-1}, E_{2} \in \Psi_{\mathrm{b}}^{m_{0}-2}$ and $\mathrm{WF}_{\mathrm{b}}^{\prime}\left(E_{1}^{\prime \prime}\right) \cap \Gamma=\emptyset$; therefore, the first part of the proof with $P$ and $u$ replaced by $P \Lambda^{+}$and $\Lambda^{-} u$, respectively, applies. The proof of the theorem in the case $r<-\sup _{\Gamma} \rho^{-m+1} e_{1} / c_{\partial}$ is complete.

When the role of $\Gamma_{+}$and $\Gamma_{-}$is reversed, there is an overall sign change, and we thus get a advantageous (now meaning positive) contribution to the subprincipal part of the conjugated operator $P_{r}$ for $r>-\inf _{\Gamma} \rho^{-m+1} e_{1} / c_{\partial}$; the rest of the argument is unchanged.

Remark 4.6. As in the radial point estimate, see Remark 4.4, the assumption that an open neighborhood of the trapped set embeds into $\overline{\mathbb{R}_{+}^{n}}$ as an open set, true 
for Kerr-de Sitter space but false for general operators on a manifold $M$, can be removed easily if we assume $\widetilde{R} \in H_{\mathrm{b}}^{s-1, \alpha} \Psi_{\mathrm{b}}^{m-2}(M)$ : To see this, one needs to make sense of the commutator computation involving the non-smooth part $\widetilde{P}$ of $P$. We begin by establishing a bound on $\widetilde{P}_{m, r}-\widetilde{P}_{m, r}^{*} \in \mathcal{L}\left(H_{\mathrm{b}}^{(m-1) / 2}(M), H_{\mathrm{b}}^{-(m-1) / 2}(M)\right)$, which we obtain by writing (we drop the subscripts for brevity)

$$
\widetilde{P}-\widetilde{P}^{*}=\sum_{j} \phi_{j} \widetilde{P}-\widetilde{P}^{*} \phi_{j}
$$

where $\left\{\phi_{j}\right\}$ is a partition of unity on $M$ subordinated to a cover by local coordinate charts; then, writing $\phi^{(0)}=\phi_{j}$ for any fixed $j$, and choosing $\phi^{(1)} \in \mathcal{C}_{\mathrm{c}}^{\infty}(M)$ supported in the same coordinate chart as $\phi^{(0)}$ and identically 1 near $\operatorname{supp} \phi^{(0)}$, we further write

$$
\begin{aligned}
\phi^{(0)} \widetilde{P}-\widetilde{P}^{*} \phi^{(0)}= & \left(\phi^{(0)} \widetilde{P} \phi^{(1)}-\left(\phi^{(0)} \widetilde{P} \phi^{(1)}\right)^{*}\right) \\
& +\left(\phi^{(0)} \widetilde{P}\left(1-\phi^{(1)}\right)-\left(1-\phi^{(1)}\right) \widetilde{P}^{*} \phi^{(0)}\right),
\end{aligned}
$$

where the first term is a bounded operator between the aforementioned spaces by the local (in $\overline{\mathbb{R}_{+}^{n}}$ ) argument, while the second term belongs to the class $H_{\mathrm{b}}^{s} \Psi_{\mathrm{b}}^{-\infty}+$ $\Psi_{\mathrm{b}}^{-\infty} H_{\mathrm{b}}^{s}$ and is thus bounded on the relevant spaces as well. Here, we remark that the regularity requirements on $s$ for $H_{\mathrm{b}}^{s} \Psi_{\mathrm{b}}^{-\infty}$ to map $H_{\mathrm{b}}^{(m-1) / 2}$ into $H_{\mathrm{b}}^{-(m-1) / 2}$ are the same as or weaker than the requirements on $s$ from the local argument, while there are no requirements other than, say, $s>n / 2$, in order to have $\Psi_{\mathrm{b}}^{-\infty} H_{\mathrm{b}}^{s}$ map $H_{\mathrm{b}}^{\sigma_{1}}$ into $H_{\mathrm{b}}^{\sigma_{2}}$ for any $\sigma_{1}, \sigma_{2} \in \mathbb{R}, \sigma_{1} \geq 0$, since $H_{\mathrm{b}}^{s} \cdot H_{\mathrm{b}}^{\sigma_{1}} \subset H_{\mathrm{b}}^{\min \left(s, \sigma_{1}\right)}$ (with a tame bound for the product) gets mapped into $H_{\mathrm{b}}^{\infty} \subset H_{\mathrm{b}}^{\sigma_{2}}$ by $\Psi_{\mathrm{b}}^{-\infty}$.

Similarly, one can introduce localizers in all commutator estimates, with the resulting error terms satisfying tame bounds by the a priori assumptions on $u$; for example, the pairing $\left\langle J^{-} A^{*}[P, A] u, u\right\rangle$ (with $J^{-}$as in the proof) is equal to an error term (generated by the localizers as above) plus the sum of

$$
\left\langle\phi^{(0)} J^{-} A^{*} \phi^{(1)}\left[\phi^{(2)} P \phi^{(3)}, \phi^{(4)} A \phi^{(5)}\right] \phi^{(6)} u, \phi^{(7)} u\right\rangle,
$$

where $\phi^{(0)}$ runs over a partition of unity on $M$ subordinated to a cover by coordinate charts, and where for $k=1, \ldots, 7, \phi^{(k)} \in \mathcal{C}_{\mathrm{c}}^{\infty}(M)$ is a cutoff, with $\phi^{(k)} \equiv 1$ on $\operatorname{supp} \phi^{(k-1)}$, and $\operatorname{supp} \phi^{(k)}$ contained in the same coordinate chart as $\phi^{(0)}$; the error term can be estimated by a tame bound involving the a priori regularity of $u$. All operators now have Schwartz kernels supported within a single coordinate chart, and all functions on which the operators act have support in the same coordinate chart, hence the local non-smooth theory, as used in the local positive commutator estimate, shows that the operator acting on $u$ in the above pairing is equal to

$$
\mathrm{Op}\left(\phi^{(0)} j^{-} a H_{\phi^{(2)} p} \phi^{(4)} a\right) \phi^{(6)}=\mathrm{Op}\left(\phi^{(0)} j^{-} a H_{p} a\right) \phi^{(6)} .
$$

At this point, one can plug in the terms of the symbolic positive commutator calculation of $a H_{p} a$, all of which now get multiplied by $\phi^{(0)}$. Since we have an invariant calculus for smooth operators, summing over the partition of unity (of which $\phi^{(0)}$ is a member) recovers the usual positive commutator calculation, with a non-smooth error term of the form $\operatorname{Op}\left(-\phi^{(0)} j^{-} f\right) \phi^{(6)}$ coming from each coordinate chart; but each of these error terms separately has a sign (since $f$ does) and is thus controlled by the sharp Gårding inequality for non-smooth operators in a coordinate chart. 
In particular, we see that there are no further regularity requirements for the invariant estimate, the requirements given in the statement of the theorem being sufficient.

4.4. Trapping estimates at normally hyperbolic trapping. Complementing the results above on negatively weighted spaces, we recall results of Dyatlov from $[22,23]$ on semiclassical estimates for smooth operators at normally hyperbolic trapping, which via the Mellin transform correspond to estimates on non-negatively weighted spaces. Here we present the results in the semiclassical setting, then in $\S 5.1$ we relate this to the solvability of linear equations with Sobolev coefficients in Theorem 5.5 and Theorem 5.6. The results of Dyatlov we use followed and built on the breakthrough earlier work of Wunsch and Zworski [53], as well as Nonnenmacher and Zworski [43]. (In the analytic category there were earlier results of Gérard and Sjöstrand [27].) The advantage of the framework of [22, 23] for us, especially as espoused in [23], is the explicit size of the 'spectral gap' (discussed below), which was also shown by Nonnenmacher and Zworski [43], the explicit inclusion of a subprincipal term of the correct sign, and the relative ease with which the parameter dependence can be analyzed.

We first recall the semiclassical setting of [23] for $^{12}$

$$
\widetilde{P}_{0}=\widetilde{P}_{0}(h), \widetilde{Q}_{0}=\widetilde{Q}_{0}(h) \in \Psi_{\hbar}^{m}(X),
$$

both formally self-adjoint, with $\widetilde{Q}_{0}$ having non-negative principal symbol, $\widetilde{P}_{0}-i \widetilde{Q}_{0}$ elliptic in the standard sense. In fact, the results in [23] are stated in the special case $m=0$, but by ellipticity of $\widetilde{P}_{0}-i \widetilde{Q}_{0}$ in the standard sense, it is straightforward to allow general $m$; see also the remark [23, bottom of p. 2]. The main assumption, see $[23$, p. 3$]$, then is that $\widetilde{P}_{0}$ has normally hyperbolic trapping semiclassically at $\widetilde{\Gamma} \subset T^{*} X$ compact, ${ }^{13}$ with all bicharacteristics of $\widetilde{P}_{0}$, except those in the stable $(-)$ and unstable $(+)$ submanifolds $\widetilde{\Gamma}_{ \pm}$, entering the elliptic set of $\widetilde{Q}_{0}$ in the forward (the exception being for only the - sign), resp. backward $(+)$ direction, and $\gamma<\nu_{\min } / 2$, where $\nu_{\text {min }}>0$ is the minimal normal expansion rate of the flow at $\widetilde{\Gamma}$, discussed above and in (4.27). If $\widetilde{Q}_{0}$ is microlocally in $h \Psi_{\hbar}(X)$ near $\widetilde{\Gamma}$, with $h^{-1} \widetilde{Q}_{0}$ having a non-negative principal symbol there, the Theorem 1 in [23] shows that there is $h_{0}>0$ such that for $\operatorname{Im} z>-\gamma$,

$$
\|v\|_{H_{h}^{s}} \lesssim h^{-2}\left\|\left(\widetilde{P}_{0}-i \widetilde{Q}_{0}-h z\right) v\right\|_{H_{h}^{s-m}}, h<h_{0} .
$$

In view of $\widetilde{\Gamma}$ lying in a compact subset of $T^{*} X$, the order $s$ is irrelevant in the sense that the estimate for one value of $s$ implies that for all other via elliptic estimates; thus, one may just take $s=0$, and even replace $s-m$ by 0 , in which case this is an $L^{2}$-estimate, as stated explicitly in [23]. We emphasize that while parts of [22] use the model forms of operators, this is no longer the case in [23], thus apart from the already discussed modifications for the differential operator and Sobolev space orders, the results apply verbatim in our setting, without the necessity to

\footnotetext{
${ }^{12}$ We write ' $h$ ' for the semiclassical parameter, and use the subscript ' $\hbar$ ' for spaces of semiclassical operators, symbols and distributions.

${ }^{13}$ Our $\widetilde{\Gamma}$ is the intersection of what is called $K$ in [23] with the semiclassical characteristic set of $P$, and similarly our $\widetilde{\Gamma}_{ \pm}$are the intersection of what is called $\Gamma_{ \pm}$in [23] with the characteristic set of $P$.
} 
arrange these model forms. Furthermore, [22, Lemma 5.1], which we use below, is also directly applicable in our setting.

Suppose now that one has a family of operators $\widetilde{P}_{0}(\omega)$ depending on another parameter, $\omega$, in a compact space $S$, with $\widetilde{P}_{0}, \widetilde{Q}_{0}$ depending continuously on $\omega$, with values in $\Psi_{\hbar}^{m}(X)$, satisfying all of the assumptions listed above. Suppose moreover that this family is uniformly normally hyperbolic, i.e. satisfies the normally hyperbolic assumptions with $\widetilde{\Gamma}, \widetilde{\Gamma}_{ \pm}$continuously depending on $\omega$ in the $\mathcal{C}^{\infty}$ topology, and uniform bounds for the normal expansion rates in the sense that both $\nu$ and the constant $C^{\prime}$ in

$$
\sup _{\rho \in \Gamma}\left\|\left.d e^{\mp t H_{p}}(\rho)\right|_{\mathcal{V}_{ \pm}}\right\| \leq C^{\prime} e^{-\nu t}, t \geq 0
$$

with $\mathcal{V}_{ \pm}$the unstable and stable normal tangent bundles at $\Gamma$, can be chosen uniformly (cf. [22, Equation (5.1)]); $\nu_{\min }$ is then the sup of these possible choices of $\nu$. (Note that since the trapped set dynamics involves arbitrarily large times, it is not automatically stable, unlike the dynamics away from the trapped set.) In this case the implied constant $C$ in (4.26), as well as $h_{0}$, is uniform in $\omega$. Note that $r$-normal hyperbolicity for every $r$ implies the local uniformity of the normal dynamics by structural stability; see $[53, \S 1]$ and $[22, \S 5.2]$.

To see this uniformity in $C$, we first point out that in [22, Lemma 5.1] the construction of $\phi_{ \pm}$can be done continuously with values in $\mathcal{C}^{\infty}$ in this case. Then in the proof of (4.26) given in [23], we only need to observe that the direct estimates provided are certainly uniform in this case for families $\widetilde{P}_{0}, \widetilde{Q}_{0}$, and furthermore for the main argument, using semiclassical defect measures, one can pass to an $L^{2}$-bounded subsequence $u_{j}$ such that $\left(\widetilde{P}_{0}\left(\omega_{j}\right)-i \widetilde{Q}_{0}\left(\omega_{j}\right)-\lambda_{j}\right) u_{j}=O\left(h^{2}\right)$, with $\omega_{j} \rightarrow \omega$ for some $\omega \in S$ in addition to $h^{-1} \lambda_{j}$ converging to some $\tilde{\lambda}$. Concretely, all results in $[23, \S 2]$ are based on elliptic or (positive) commutator identities or estimates which are uniform in this setting. In particular, [23, Lemma 2.3] is valid with $P_{j}=P\left(\omega_{j}\right) \rightarrow P, W_{j}=W\left(\omega_{j}\right) \rightarrow W$ with convergence in $\Psi_{\hbar}(X)$. (This uses that one can take $A_{j}\left(h_{j}\right)$ in Definition 2.1, with $A_{j} \rightarrow A$, since the difference between $A_{j}\left(h_{j}\right)$ and $A\left(h_{j}\right)$ is bounded by a constant times the squared $L^{2}$-norm of $u_{j}$ times the operator norm bound of $A_{j}\left(h_{j}\right)-A\left(h_{j}\right)$, with the latter going to 0.) Then with $\Theta_{+, j}$ in place of $\Theta_{+}$, one still gets Lemma 3.1, which means that Lemma 3.2 still holds with $\phi_{+}$(the limiting $\phi_{+, j}$ ) using Lemma 2.3. Then the displayed equation above [23, Equation (3.9)] still holds with the limiting $\widetilde{P}_{0}=\widetilde{P}_{0}(\omega)$, again by Lemma 2.3 , and then one can finish the argument as in [23]. With this modification, one obtains the desired uniformity. This in particular allows one to apply (4.26) even if $\widetilde{P}_{0}$ and $\widetilde{Q}_{0}$ depend on $z$ (in a manner consistent with the other requirements), which can also be dealt with more directly using the model form in [22, Lemma 4.3]. It also allows for uniform estimates for families depending on a small parameter in $\mathbb{C}$, denoted by $v_{0}$ below, needed in $\S 5$.

Allowing $\widetilde{P}_{0}$ and $\widetilde{Q}_{0}$ depending on $z$ means, in particular, that we can replace the requirement on $h^{-1} \widetilde{Q}_{0}$ by the principal symbol of $h^{-1} \widetilde{Q}_{0}$ being $>-\beta, \beta<\nu_{\min } / 2$, and drop $z$, so one has

$$
\|v\|_{H_{h}^{s}} \lesssim h^{-2}\left\|\left(\widetilde{P}_{0}-i \widetilde{Q}_{0}\right) v\right\|_{H_{h}^{s-m}}, h<h_{0}
$$


At this point it is convenient to rewrite this estimate, removing $\widetilde{Q}_{0}$ from the right hand side at the cost (or benefit!) of making it microlocal. ${ }^{14}$ From here on it is convenient to change the conventions and not require that $\widetilde{P}_{0}$ is formally selfadjoint (though it is at the principal symbol level, namely it has a real principal symbol); translating back into the previous notation, one would replace $\widetilde{P}_{0}$ by its (formally) self-adjoint part, and absorb its skew-adjoint part into $\widetilde{Q}_{0}$. Namely, we have

Theorem 4.7. Suppose $\widetilde{P}_{0}$ satisfies the above assumptions, in particular the semiclassical principal symbol of $\frac{1}{2 i h}\left(\widetilde{P}_{0}-\widetilde{P}_{0}^{*}\right)$ being $<\beta<\nu_{\min } / 2$ at $\widetilde{\Gamma} \cdot{ }^{15}$ With $\widetilde{B}_{j}$ analogous to Theorem 4.5, with wave front set sufficiently close to $\widetilde{\Gamma}$, we have, for sufficiently small $h>0$ and for all $N$ and $s_{0}$,

$$
\left\|\widetilde{B}_{0} u\right\|_{H_{h}^{s}} \lesssim h^{-2}\left\|\widetilde{B}_{1} \widetilde{P}_{0} u\right\|_{H_{h}^{s-m+1}}+h^{-1}\left\|\widetilde{B}_{2} u\right\|_{H_{h}^{s}}+h^{N}\|u\|_{H_{h}^{s_{0}}} .
$$

Note that the differential orders are actually irrelevant here due to wave front set conditions.

Proof. Take $\widetilde{Q}_{0} \in \Psi_{\hbar}^{0}(X)$ with non-negative principal symbol such that $\mathrm{WF}_{\hbar}^{\prime}\left(\widetilde{Q}_{0}\right)$ is disjoint from $\mathrm{WF}_{\hbar}^{\prime}\left(\widetilde{B}_{0}\right)$, and so that all backward bicharacteristics from points not in $\widetilde{\Gamma}_{+}$, as well as forward bicharacteristics from points not in $\widetilde{\Gamma}_{-}$, reach the elliptic set of $\widetilde{Q}_{0}$, and with $\widetilde{B}_{1}$ elliptic on the complement of the elliptic set of $\widetilde{Q}_{0}$. Let $\widetilde{B}_{3} \in \Psi_{\hbar}^{0}(X)$ to be such that $\mathrm{WF}_{\hbar}^{\prime}\left(I-\widetilde{B}_{3}\right)$ is disjoint from $\mathrm{WF}_{\hbar}^{\prime}\left(\widetilde{B}_{0}\right)$ but $\mathrm{WF}_{\hbar}^{\prime}\left(\widetilde{Q}_{0}\right) \cap \mathrm{WF}_{\hbar}^{\prime}\left(\widetilde{B}_{3}\right)=\emptyset$. Let $\widetilde{A}_{+} \in \Psi_{\hbar}^{0}(X)$ have wave front set near $\widetilde{\Gamma}_{+}$, with

$$
\mathrm{WF}_{\hbar}^{\prime}\left(I-\widetilde{A}_{+}\right) \cap \mathrm{WF}_{\hbar}^{\prime}\left(\widetilde{B}_{3}\right) \cap \widetilde{\Gamma}_{+}=\emptyset
$$

and with

$$
\mathrm{WF}_{\hbar}^{\prime}\left(\widetilde{A}_{+}\right) \cap \mathrm{WF}_{\hbar}^{\prime}\left(I-\widetilde{B}_{3}\right) \cap \widetilde{\Gamma}_{-}=\emptyset,
$$

and with no backward bicharacteristic from $\operatorname{WF}_{\hbar}^{\prime}\left(\widetilde{B}_{0}\right)$ reaching

$$
\mathrm{WF}_{\hbar}^{\prime}\left(\widetilde{A}_{+}\right) \cap \mathrm{WF}_{\hbar}^{\prime}\left(I-\widetilde{B}_{3}\right) \cap \widetilde{\Gamma}_{+} .
$$

Take $\widetilde{Q}_{1}$ elliptic on $\widetilde{\Gamma}$, with $\mathrm{WF}_{\hbar}^{\prime}\left(\widetilde{Q}_{1}\right) \cap \mathrm{WF}_{\hbar}^{\prime}\left(I-\widetilde{B}_{3}\right)=\emptyset$, again with non-negative principal symbol, with no backward bicharacteristic from $\mathrm{WF}_{\hbar}^{\prime}\left(\widetilde{Q}_{1}\right)$ reaching

$$
\mathrm{WF}_{\hbar}^{\prime}\left(\widetilde{A}_{+}\right) \cap \mathrm{WF}_{\hbar}^{\prime}\left(I-\widetilde{B}_{3}\right) .
$$

Thus, all backward and forward bicharacteristics of $\widetilde{P}_{0}$ reach the elliptic set of $\widetilde{Q}_{1}$ or $\widetilde{Q}_{0}$. See Figure 5 for the setup.

Then

$$
\left(\widetilde{P}_{0}-i \widetilde{Q}_{0}\right) \widetilde{B}_{3} u=\widetilde{B}_{3} \widetilde{P}_{0} u+\widetilde{A}_{+}\left[\widetilde{P}_{0}, \widetilde{B}_{3}\right] u+\left(I-\widetilde{A}_{+}\right)\left[\widetilde{P}_{0}, \widetilde{B}_{3}\right] u-i \widetilde{Q}_{0} \widetilde{B}_{3} u
$$

\footnotetext{
${ }^{14} \mathrm{An}$ alternative would be using the gluing result of Datchev and Vasy [17], which is closely related in approach.

${ }^{15}$ The apparent sign change here as compared to before comes from the fact that for formally self-adjoint $\widetilde{P}_{0}, \widetilde{Q}_{0}$, one has $\frac{1}{2 i h}\left(\left(\widetilde{P}_{0}-i \widetilde{Q}_{0}\right)-\left(\widetilde{P}_{0}-i \widetilde{Q}_{0}\right)^{*}\right)=-h^{-1} \widetilde{Q}_{0}$; notice the minus sign on the right hand side.
} 


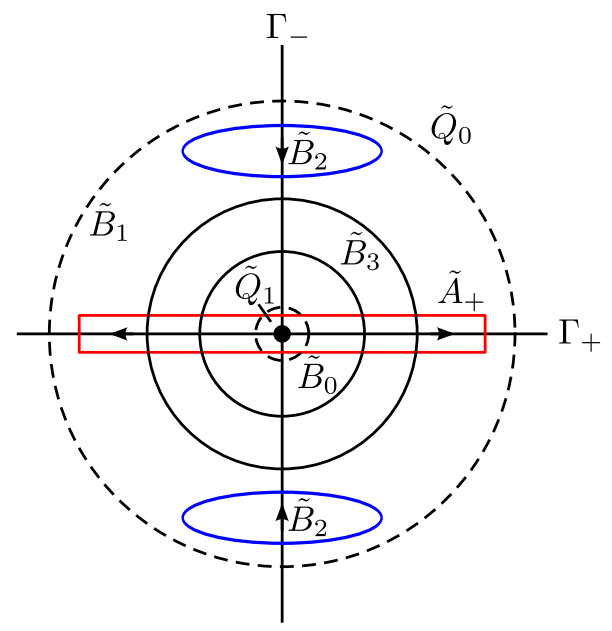

FiguRE 5. Setup for the proof of the microlocalized normally hyperbolic trapping estimate (4.29): Indicated are the backward and forward trapped sets $\Gamma_{+}$and $\Gamma_{-}$, respectively, which intersect at $\Gamma$ (large dot). We use complex absorbing potentials $\widetilde{Q}_{0}$ (with $\mathrm{WF}_{\hbar}^{\prime}\left(\widetilde{Q}_{0}\right)$ outside the large dashed circle) and $\widetilde{Q}_{1}\left(\right.$ with $\mathrm{WF}_{\hbar}^{\prime}\left(\widetilde{Q}_{1}\right)$ inside the small dashed circle). We obtain an estimate for $\widetilde{B}_{0} u$ by combining (4.28) with microlocal propagation from the elliptic set of $\widetilde{B}_{2}$.

SO

$$
\begin{aligned}
\widetilde{B}_{0} u= & \widetilde{B}_{0} \widetilde{B}_{3} u+\widetilde{B}_{0}\left(I-\widetilde{B}_{3}\right) u \\
= & \widetilde{B}_{0}\left(\widetilde{P}_{0}-i \widetilde{Q}_{0}\right)^{-1} \widetilde{B}_{3} \widetilde{P}_{0} u+\widetilde{B}_{0}\left(\widetilde{P}_{0}-i \widetilde{Q}_{0}\right)^{-1} \widetilde{A}_{+}\left[\widetilde{P}_{0}, \widetilde{B}_{3}\right] u \\
& \quad+\widetilde{B}_{0}\left(\widetilde{P}_{0}-i \widetilde{Q}_{0}\right)^{-1}\left(I-\widetilde{A}_{+}\right)\left[\widetilde{P}_{0}, \widetilde{B}_{3}\right] u \\
& \quad-i \widetilde{B}_{0}\left(\widetilde{P}_{0}-i \widetilde{Q}_{0}\right)^{-1} \widetilde{Q}_{0} \widetilde{B}_{3} u+\widetilde{B}_{0}\left(I-\widetilde{B}_{3}\right) u,
\end{aligned}
$$

and by (4.28), for $h<h_{0}$,

$$
\left\|\left(\widetilde{P}_{0}-i \widetilde{Q}_{0}\right)^{-1} \widetilde{B}_{3} \widetilde{P}_{0} u\right\|_{H_{h}^{s}} \lesssim h^{-2}\left\|\widetilde{B}_{3} \widetilde{P}_{0} u\right\|_{H_{h}^{s-m}} .
$$

Now, $\widetilde{Q}_{0} \widetilde{B}_{3}, \widetilde{B}_{0}\left(I-\widetilde{B}_{3}\right) \in h^{\infty} \Psi_{\hbar}^{-\infty}(X)$, so the corresponding terms in (4.30) can be absorbed into $h^{N}\|u\|_{H_{h}^{s_{0}}}$. On the other hand, since $\mathrm{WF}_{\hbar}^{\prime}\left(\left(I-\widetilde{A}_{+}\right)\left[\widetilde{P}_{0}, \widetilde{B}_{3}\right]\right)$ is disjoint from $\widetilde{\Gamma}_{+}$, the backward bicharacteristics from it reach the elliptic set of $\widetilde{B}_{2}$, and so we have the microlocal real principal type estimate for $u$ :

$$
\left\|\left(I-\widetilde{A}_{+}\right)\left[\widetilde{P}_{0}, \widetilde{B}_{3}\right] u\right\|_{H_{h}^{s-m}} \lesssim h\left\|\widetilde{B}_{2} u\right\|_{H_{h}^{s-1}}+\left\|\widetilde{B}_{1} \widetilde{P}_{0} u\right\|_{H_{h}^{s-m}}
$$

as $\left(I-\widetilde{A}_{+}\right)\left[\widetilde{P}_{0}, \widetilde{B}_{3}\right] \in h \Psi_{\hbar}^{m-1}(X)$, so by $(4.28)$,

$$
\left\|\left(\widetilde{P}_{0}-i \widetilde{Q}_{0}\right)^{-1}\left(I-\widetilde{A}_{+}\right)\left[\widetilde{P}_{0}, \widetilde{B}_{3}\right] u\right\|_{H_{h}^{s}} \lesssim h^{-1}\left\|\widetilde{B}_{2} u\right\|_{H_{h}^{s-1}}+h^{-2}\left\|\widetilde{B}_{1} \widetilde{P}_{0} u\right\|_{H_{h}^{s-m}} .
$$

Thus, (4.29) follows if we can estimate $\left\|\widetilde{B}_{0}\left(\widetilde{P}_{0}-i \widetilde{Q}_{0}\right)^{-1} \widetilde{A}_{+}\left[\widetilde{P}_{0}, \widetilde{B}_{3}\right] u\right\|_{H_{h}^{s}}$. Now, $\mathrm{WF}_{\hbar}^{\prime}\left(\widetilde{A}_{+}\left[\widetilde{P}_{0}, \widetilde{B}_{3}\right]\right) \cap \widetilde{\Gamma}_{-}=\emptyset$ by arrangement. In order to microlocalize, we now 
introduce a nontrapping model, $\widetilde{P}_{0}-i\left(\widetilde{Q}_{0}+\widetilde{Q}_{1}\right)$. We claim that

$$
v=\left(\widetilde{P}_{0}-i\left(\widetilde{Q}_{0}+\widetilde{Q}_{1}\right)\right)^{-1} \widetilde{A}_{+}\left[\widetilde{P}_{0}, \widetilde{B}_{3}\right] u-\left(\widetilde{P}_{0}-i \widetilde{Q}_{0}\right)^{-1} \widetilde{A}_{+}\left[\widetilde{P}_{0}, \widetilde{B}_{3}\right] u
$$

satisfies

$$
\|v\|_{H_{h}^{s^{\prime}}} \lesssim h^{N}\|u\|_{H_{h}^{s_{0}}}
$$

for all $s^{\prime}, N$. Notice that for any $s^{\prime \prime}$ one certainly has

$$
\|v\|_{H_{h}^{s^{\prime \prime}}} \lesssim h^{-1}\|u\|_{H_{h}^{s^{\prime \prime}-1}}
$$

by (4.28) plus its non-trapping analogue. To see (4.31), notice that

$$
\left(\widetilde{P}_{0}-i \widetilde{Q}_{0}\right) v=i \widetilde{Q}_{1}\left(\widetilde{P}_{0}-i\left(\widetilde{Q}_{0}+\widetilde{Q}_{1}\right)\right)^{-1} \widetilde{A}_{+}\left[\widetilde{P}_{0}, \widetilde{B}_{3}\right] u
$$

so by (4.28), with $s_{0}$ replaced by any $s_{0}^{\prime}$ (since $s_{0}$ was arbitrary), and for any $N$,

$$
\|v\|_{H_{h}^{s^{\prime}}} \lesssim h^{-2}\left\|\widetilde{Q}_{1}\left(\widetilde{P}_{0}-i\left(\widetilde{Q}_{0}+\widetilde{Q}_{1}\right)\right)^{-1} \widetilde{A}_{+}\left[\widetilde{P}_{0}, \widetilde{B}_{3}\right] u\right\|_{H_{h}^{s^{\prime}-m}} \lesssim h^{N}\|u\|_{H_{h}^{s_{0}}},
$$

since $\widetilde{P}_{0}-i\left(\widetilde{Q}_{0}+\widetilde{Q}_{1}\right)$ is non-trapping, hence $\left(\widetilde{P}_{0}-i\left(\widetilde{Q}_{0}+\widetilde{Q}_{1}\right)\right)^{-1}$ propagates semiclassical wave front sets along forward bicharacteristics, and no backward bicharacteristic from $\mathrm{WF}_{\hbar}^{\prime}\left(\widetilde{Q}_{1}\right)$ can reach $\mathrm{WF}_{\hbar}^{\prime}\left(\widetilde{A}_{+}\left[\widetilde{P}_{0}, \widetilde{B}_{3}\right]\right) \subset \mathrm{WF}_{\hbar}^{\prime}\left(\widetilde{A}_{+}\right) \cap \mathrm{WF}_{\hbar}^{\prime}\left(I-\widetilde{B}_{3}\right)$, proving the claim. Then, since backward bicharacteristics from $\mathrm{WF}_{\hbar}^{\prime}\left(\widetilde{B}_{0}\right)$ do not encounter $\mathrm{WF}_{\hbar}^{\prime}\left(\widetilde{A}_{+}\left[\widetilde{P}_{0}, \widetilde{B}_{3}\right]\right) \cap \widetilde{\Gamma}_{+}$before reaching the elliptic set of $\widetilde{Q}_{0}$ or $\widetilde{Q}_{1}$, we conclude that

$$
\begin{aligned}
& \left\|\widetilde{B}_{0}\left(\widetilde{P}_{0}-i \widetilde{Q}_{0}\right)^{-1} \widetilde{A}_{+}\left[\widetilde{P}_{0}, \widetilde{B}_{3}\right] u\right\|_{H_{h}^{s}} \\
& \quad \leq\left\|\widetilde{B}_{0}\left(\widetilde{P}_{0}-i \widetilde{Q}_{0}-i \widetilde{Q}_{1}\right)^{-1} \widetilde{A}_{+}\left[\widetilde{P}_{0}, \widetilde{B}_{3}\right] u\right\|_{H_{h}^{s}}+\left\|\widetilde{B}_{0} v\right\|_{H_{h}^{s}} \\
& \quad \lesssim h\left\|\widetilde{B}_{2} u\right\|_{H_{h}^{s}}+\left\|\widetilde{B}_{1} \widetilde{P}_{0} u\right\|_{H_{h}^{s-m+1}}+h^{N}\|u\|_{H_{h}^{s_{0}}} .
\end{aligned}
$$

This proves (4.29), and thus the theorem.

\section{Quasilinear wave and Klein-Gordon equations}

5.1. Forward solution operators. We now generalize the setting considered in $[30, \S 7.2]$ for the study of quasilinear equations on static asymptotically de Sitter spaces to allow for normally hyperbolic trapping, as discussed in the previous section.

For simplicity, we first describe the scalar setup: Working on a compact manifold $M$ with boundary $X$, we assume that the operator $P$ is of the form $P=P_{0}+\widetilde{P}$, continuously depending on a small parameter

$$
v=\left(v_{0}, \widetilde{v}\right) \in \mathcal{X}^{\widetilde{s}, \alpha}:=\mathbb{C} \oplus H_{\mathrm{b}}^{\widetilde{s}, \alpha}, \quad \alpha>0,
$$

where

$$
\begin{aligned}
& P_{0}=P_{0}\left(v_{0}\right)=\square_{g\left(v_{0}\right)}+L\left(v_{0}\right) \in \operatorname{Diff}_{\mathrm{b}}^{2}(M), \\
& L\left(v_{0}\right) \in \operatorname{Diff}_{\mathrm{b}}^{1}(M), \quad L(0)-L(0)^{*} \in \operatorname{Diff}_{\mathrm{b}}^{0}(M), \\
& \widetilde{P}=\widetilde{P}(v) \in H_{\mathrm{b}}^{\widetilde{s}, \alpha} \operatorname{Diff}_{\mathrm{b}}^{2}(M)+H_{\mathrm{b}}^{\widetilde{s}-1, \alpha} \operatorname{Diff}_{\mathrm{b}}^{1}(M)
\end{aligned}
$$

for a smooth b-metric $g$ on $M$ that continuously depends on one complex parameter; here, we identify $v=\left(v_{0}, \widetilde{v}\right)$ with the distribution $v_{0} \chi+\widetilde{v}$ on $M$, where $\chi \in \mathcal{C}^{\infty}(M)$ is a cutoff, identically 1 near $X$. An example to keep in mind for the remainder of the section is the wave operator on an (asymptotically) Kerr-de Sitter space or a metric $H_{\mathrm{b}}^{\widetilde{s}+1, \alpha}$-perturbation thereof. We assume: 
(1) The characteristic set $\Sigma \subset{ }^{\mathrm{b}} S_{X}^{*} M$ of $P_{0}$ has the form $\Sigma=\Sigma_{+} \cup \Sigma_{-}$with $\Sigma_{ \pm}$a union of connected components of $\Sigma$,

(2) $P_{0}$ has normally hyperbolic trapping at $\Gamma^{ \pm} \subset \Sigma_{ \pm}$for small $v_{0}$, as detailed in assumptions (1)-(10) in $\S 4.2$,

(3) $P_{0}$ has radial sets $L_{ \pm} \subset{ }^{\mathrm{b}} S_{X}^{*} M$, which, in appropriate directions transverse to $L_{ \pm}$, are sources $(-) / \operatorname{sinks}(+)$ for the null-bicharacteristic flow within ${ }^{\mathrm{b}} S_{X}^{*} M$, with a one-dimensional stable/unstable manifold intersecting ${ }^{\mathrm{b}} S_{X}^{*} M$ transversally; for details, see [30, 86.4$]$. In particular, there are $\beta_{0}, \widetilde{\beta} \in \mathcal{C}^{\infty}\left(L_{ \pm}\right), \beta_{0}, \widetilde{\beta}>0$, such that for a homogeneous degree -1 boundary defining function $\rho$ of fiber infinity in ${ }^{\mathrm{b}} \bar{T}^{*} M$ and with $V=\rho H_{p_{0}}$,

$$
\left.\rho^{-1} V \rho\right|_{L_{ \pm}}=\mp \beta_{0}, \quad-\left.x^{-1} V x\right|_{L_{ \pm}}=\mp \widetilde{\beta} \beta_{0} .
$$

We will set up initial value problems by introducing artificial boundaries as in $[30,33]$ : We denote by $\mathfrak{t}_{1}$ and $\mathfrak{t}_{2}$ two smooth functions on $M$ and put ${ }^{16}$

$$
\Omega=\mathfrak{t}_{1}^{-1}([0, \infty)) \cap \mathfrak{t}_{2}^{-1}([0, \infty)),
$$

where we assume that:

(4) $\Omega$ is compact,

(5) putting $H_{j}:=\mathfrak{t}_{j}^{-1}(0)$, the $H_{j}$ intersect the boundary $\partial M$ transversally, and $H_{1}$ and $H_{2}$ intersect only in the interior of $M$, and they do so transversally,

(6) the differentials of $\mathfrak{t}_{1}$ and $\mathfrak{t}_{2}$ have opposite timelike characters near their respective zero sets within $\Omega$; more specifically, $d \mathfrak{t}_{1}$ is future timelike, $d \mathfrak{t}_{2}$ past timelike,

(7) there is a boundary defining function $x$ of $M$ such that $d x / x$ is timelike on $\Omega \cap \partial M$ with timelike character opposite to the one of $d \mathfrak{t}_{1}$, i.e. $d x / x$ is past oriented,

(8) the dynamical structure of the null-geodesic flow (within ${ }^{\mathrm{b}} S_{\Omega}^{*} M$ ) of the metric $g$ is as follows: All bicharacteristics in $\Sigma_{\Omega}:=\Sigma \cap{ }^{\mathrm{b}} S_{\Omega}^{*} M$ from any point in $\Sigma_{\Omega} \cap\left(\Sigma_{+} \backslash\left(L_{+} \cup \Gamma^{+}\right)\right)$flow (within $\left.\Sigma_{\Omega}\right)$ to ${ }^{\mathrm{b}} S_{H_{1}}^{*} M \cup L_{+} \cup \Gamma^{+}$ in the forward direction (i.e. either enter ${ }^{\mathrm{b}} S_{H_{1}}^{*} M$ in finite time or tend to the radial set $L_{+}$or the trapped set $\Gamma^{+}$) and to ${ }^{\mathrm{b}} S_{H_{2}}^{*} M \cup L_{+} \cup \Gamma^{+}$ in the backward direction, and from any point in $\Sigma_{\Omega} \cap\left(\Sigma_{-} \backslash\left(L_{-} \cup \Gamma^{-}\right)\right)$ to ${ }^{\mathrm{b}} S_{H_{2}}^{*} M \cup L_{-} \cup \Gamma^{-}$in the forward direction and to ${ }^{\mathrm{b}} S_{H_{1}}^{*} M \cup L_{-} \cup \Gamma^{-}$ in the backward direction, with tending to $\Gamma^{ \pm}$allowed in only one of the two directions. In conventional scattering theory 'non-trapping' is usually understood to mean that all bicharacteristics in the characteristic set escape to infinity; in microlocal terms [41] this means exactly that they tend to radial sets in both the forward and backward directions. Thus, in the sense of scattering theory our assumption is that the normally hyperbolic trapping describes all the trapping present here.

Recall the space $H_{\mathrm{b}}^{s, r}(\Omega)^{\bullet},-$ of distributions which are supported $(\bullet)$ at the 'artificial' boundary hypersurface $H_{1}$ and extendible $(-)$ at $H_{2}$, and the other way around for $H_{\mathrm{b}}^{s, r}(\Omega)^{-, \bullet}$, see [34, Appendix B]: Thus, elements of $H_{\mathrm{b}}^{s, r}(\Omega)^{\bullet,-}$ are restrictions to $\Omega^{\circ}$ of functions in $H_{\mathrm{b}}^{s, r}(M)$ which are supported in the future of $H_{1}$,

\footnotetext{
${ }^{16}$ In the Kerr-de Sitter setting described in the introduction, we can take $\mathfrak{t}_{1}=1-x$, which equals $\mathfrak{t}_{1}=1-e^{-t_{*}}$ in $M^{\circ}$, and $\mathfrak{t}_{2}=\mp\left(r-\left(r_{ \pm} \pm \delta\right)\right), \delta>0$ small, near $r=r_{ \pm}$, or indeed $\mathfrak{t}_{2}=-\left(r-\left(r_{+}+\delta\right)\right)\left(r-\left(r_{-}-\delta\right)\right)$ globally, which is positive precisely in $\left(r_{-}-\delta, r_{+}+\delta\right)$.
} 
i.e. in $\mathfrak{t}_{1}^{-1}([0, \infty))$. Then we have the following global energy estimates, with the proof exactly as in the reference:

Lemma 5.1. (Cf. [30, Lemma 7.3].) Suppose $\widetilde{s}>n / 2+2$. There exists $r_{0}<0$ such that for $r \leq r_{0},-\widetilde{r} \leq r_{0}$, there is $C>0$ such that for $u \in H_{\mathrm{b}}^{2, r}(\Omega)^{\bullet},-$, $v \in H_{\mathrm{b}}^{2, \widetilde{r}}(\Omega)^{-, \bullet}$, one has

$$
\begin{aligned}
\|u\|_{H_{\mathrm{b}}^{1, r}(\Omega)} \bullet & \leq C\|P u\|_{H_{\mathrm{b}}^{0, r}(\Omega)^{\bullet,-}}, \\
\|v\|_{H_{\mathrm{b}}^{1, \tilde{r}}(\Omega)^{-}, \bullet} & \leq C\left\|P^{*} v\right\|_{H_{\mathrm{b}}^{0, \tilde{r}}(\Omega)^{-,}, \bullet}
\end{aligned}
$$

We recall from [30] that this result does not rely on the dynamical structure of $P$ at the boundary, but only on the timelike nature of $d x / x$ and of $d \mathfrak{t}_{1}$ and $d \mathfrak{t}_{2}$ near $H_{1}$ and $H_{2}$, respectively, see also [30, Remark 7.4].

Let us stress that we assume the parameter $v$ to be small so that in particular the skew-adjoint part of $P_{0}\left(v_{0}\right)$ is small and does not affect the radial point and normally hyperbolic trapping estimates which are used in what follows; the general case without symmetry assumptions on $P_{0}(0)$ will be discussed in $§ 5.4$. Using a duality argument and the tame estimates for elliptic regularity and the propagation of singularities (real principal type, radial points, normally hyperbolic trapping) given in Propositions 4.1, 4.2 and 4.3 and Theorem 4.5, we thus obtain solvability and higher regularity:

Lemma 5.2. (Cf. [30, Lemma 7.5].) Let $0 \leq s \leq \widetilde{s}$ and assume $\widetilde{s}>n / 2+6$, $s_{0}>n / 2+1 / 2$. There exists $r_{0}<0$ such that for $r \leq r_{0}$, there is $C>0$ with the following property: If $f \in H_{\mathrm{b}}^{s-1, r}(\Omega)^{\bullet,-}$, then there exists a unique $u \in H_{\mathrm{b}}^{s, r}(\Omega)^{\bullet}$,such that $P u=f$, and $u$ moreover satisfies

$$
\|u\|_{H_{\mathrm{b}}^{s, r}(\Omega) \bullet,-} \lesssim\|f\|_{H_{\mathrm{b}}^{s-1, r}(\Omega) \cdot,-}+\|f\|_{H_{\mathrm{b}}^{s_{0}, r}(\Omega)} \cdot\|v\|_{\mathcal{X}^{\tilde{s}, \alpha}} .
$$

Here, the implicit constant depends only on s and $\|v\|_{\mathcal{X}^{n / 2+6+\epsilon, \alpha}}$ for $\epsilon>0$.

Proof. The proof proceeds as the proof given in the reference, using Theorem 4.5 to obtain microlocal regularity at the trapped set. The tame estimate (5.3) in particular is obtained by iterative use of the aforementioned microlocal regularity estimates; the given bound for $s_{0}$ comes from an inspection of the norms in these estimates which correspond to the terms called $u_{*}^{\ell}$ in (4.1).

We deduce analogues of [30, Corollaries 7.6-7.7]:

Corollary 5.3. Let $0 \leq s \leq \widetilde{s}$ and assume $\widetilde{s}>n / 2+6, s_{0}>n / 2+1 / 2$. There exists $r_{0}<0$ such that for $r \leq r_{0}$, there is $C>0$ with the following property: If $u \in H_{\mathrm{b}}^{s, r}(\Omega)^{\bullet},-$ is such that $P u \in H_{\mathrm{b}}^{s-1, r}(\Omega)^{\bullet},-$, then the estimate (5.3) holds.

Corollary 5.4. Let $s_{0}>n / 2+1 / 2, s_{0} \leq s^{\prime} \leq s \leq \widetilde{s}$, and assume $\widetilde{s}>n / 2+6$; moreover, let $r<0$. Then there is $C>0$ such that the following holds: Any $u \in H_{\mathrm{b}}^{s^{\prime}, r}(\Omega)^{\bullet,-}$ with $P u \in H_{\mathrm{b}}^{s-1, r}(\Omega)^{\bullet,-}$ in fact satisfies $u \in H_{\mathrm{b}}^{s, r}(\Omega)^{\bullet,-}$, and obeys the estimate

$$
\begin{aligned}
\|u\|_{H_{\mathrm{b}}^{s, r}(\Omega) \bullet,-} \lesssim\|P u\|_{H_{\mathrm{b}}^{s-1, r}(\Omega)^{\bullet,-}}+\|u\|_{H_{\mathrm{b}}^{s^{\prime}, r}(\Omega)^{\bullet,-}} \\
+\left(\|P u\|_{H_{\mathrm{b}}^{s_{0}, r}(\Omega)^{\bullet,-}}+\|u\|_{H_{\mathrm{b}}^{s_{0}+1, r}(\Omega) \cdot,-}\right)\|v\|_{\mathcal{X}^{\tilde{s}, \alpha}} .
\end{aligned}
$$


Proof. The proof of the two corollaries is as in the cited reference. For the radial point estimate involved in the proof of Corollary 5.4, we need the additional assumption $s^{\prime}-1+\sup _{L_{ \pm}}(r \widetilde{\beta})>0$, which however is automatically satisfied since $s^{\prime} \geq 1$ and the sup is negative for $r<0$.

We now note that the Mellin transformed normal operator $\widehat{N}(P)(\sigma)$ satisfies global large parameter estimates corresponding to the semiclassical microlocal estimates of Theorem 4.7. In order to state this precisely we recall the connection between the b-structure, the normal operator (and the large parameter algebra) and the Mellin transform of the latter.

The weighted b-Sobolev spaces $H_{\mathrm{b}}^{s, \gamma}([0, \infty) \times X)$ are isometric to the large parameter Sobolev spaces on $X$ on the $\operatorname{line} \operatorname{Im} \sigma=-\gamma$ in $\mathbb{C}$ via the Mellin transform $\mathcal{M}$; see [51, Equation (3.8)]. Further, the latter can be described in terms of semiclassical Sobolev spaces, namely the restriction $r_{-\gamma} \circ \mathcal{M}$ to $\operatorname{Im} \sigma=-\gamma$ of the Mellin transform identifies $H_{\mathrm{b}}^{s, \gamma}([0, \infty) \times X)$ with

$$
\langle|\sigma|\rangle^{-s} L^{2}\left(\mathbb{R} ; H_{\langle|\sigma|\rangle^{-1}}^{s}(X)\right) ;
$$

see [51, Equation (3.9)]. Here, the norm on the space $H_{h}^{s}$ on $\mathbb{R}^{n}$ is defined by

$$
\|u\|_{H_{h}^{s}}^{2}=\int\langle h \xi\rangle^{2 s}|\widehat{u}(\xi)|^{2} d \xi, \quad h>0,
$$

and in general using a partition of unity.

Now, in order to relate b-microlocal analysis with semiclassical analysis, we first identify $\varpi+\sigma \frac{d x}{x} \in{ }^{\mathrm{b}} T^{*}([0, \infty) \times X), \varpi \in T^{*} X$, with $(\sigma, \varpi) \in \mathbb{R} \times T^{*} X$. Under the semiclassical rescaling, say by $|\sigma|^{-1}$, one identifies the latter with $h=|\sigma|^{-1}, \widetilde{\varpi}=$ $|\sigma|^{-1} \varpi$. In particular, if a conic set is disjoint from $T^{*} X$ in ${ }^{\mathrm{b}} T^{*}([0, \infty) \times X)$, then its image under the semiclassical identification lies in a compact subset of $T^{*} X$. Thus, for $B \in \Psi_{\mathrm{b}}^{0}([0, \infty) \times X)$ dilation invariant, the large parameter principal symbol and wave front set of the Mellin conjugate $\mathcal{M B M}^{-1}=\widehat{B}$ of $B$ are exactly those of $B$ under the above identification of $\varpi+\sigma \frac{d x}{x} \in{ }^{\mathrm{b}} T^{*}([0, \infty) \times X)$, $\varpi \in T^{*} X$, with $(\sigma, \varpi) \in \mathbb{R} \times T^{*} X$, and then the analogous statement also holds for $\widehat{B}$ considered as an element $\widetilde{B}$ of $\Psi_{\hbar}(X)$ under the semiclassical identification. In particular, one has, for $B \in \Psi_{\mathrm{b}}^{0}([0, \infty) \times X)$ dilation invariant, with $\operatorname{WF}_{\mathrm{b}}^{\prime}(B) \cap T^{*} X=\emptyset$, that $\widetilde{B} \in \Psi_{|\sigma|^{-1}}^{-\infty}(X)$, with semiclassical wave front set in a compact subset of $T^{*} X$. Correspondingly, for any $s_{0}$,

$$
\|B u\|_{H_{\mathrm{b}}^{s, \gamma}}^{2} \lesssim \int_{\sigma \in \mathbb{R},|\sigma|>h_{0}^{-1}}|\sigma|^{2 s}\|\widehat{B} \mathcal{M} u(.-i \gamma)\|_{L^{2}}^{2} d \sigma+\|u\|_{H_{\mathrm{b}}^{s_{0}, \gamma}}^{2}
$$

Now if $P_{0}=P_{0}\left(v_{0}\right) \in \Psi_{\mathrm{b}}^{m}(M)$, then $N\left(P_{0}\right)$ is dilation invariant on $[0, \infty) \times X$, and its conjugate by the Mellin transform is $\widehat{P}_{0}=\widehat{N}\left(P_{0}\right)$, whose rescaling $\widetilde{P}_{0}=|\sigma|^{-m} \widehat{P}_{0}$ is an element of $\Psi_{\hbar}^{m}(X)$. Further, with $P_{0}$ b-normally hyperbolic in the sense discussed above (with the convention changed regarding formal self-adjointness, as stated before Theorem 4.7), $\widetilde{P}_{0}$ is normally hyperbolic in the sense of [23]. Fix a smooth b-density on $M$ near $X$, identified with $\left[0, \epsilon_{0}\right) \times X$ as above; we require this to be of the product form $\frac{|d x|}{x} \nu, \nu$ a smooth density on $X$; we compute adjoints with respect to this density. Then for any $B \in \Psi_{\mathrm{b}}^{m}(M), \widehat{B^{*}}(\sigma)=(\widehat{B}(\bar{\sigma}))^{*}$, see $[51, \S 3.3]$ for differential operators, and by a straightforward calculation using the 
Mellin transform in general. In particular, if $B=B^{*}$, then $\widehat{B}(\sigma)=(\widehat{B}(\sigma))^{*}$ for $\sigma \in \mathbb{R}$. Relaxing (5.1) momentarily, we then assume that

$$
\frac{1}{2 i}\left(P_{0}-P_{0}^{*}\right) \in \Psi_{\mathrm{b}}^{m-1}(M),\left.\quad \sigma_{\mathrm{b}, m-1}\left(\frac{1}{2 i}\left(P_{0}-P_{0}^{*}\right)\right)\right|_{\Gamma}<|\sigma|^{m-1} \nu_{\min } / 2,
$$

with $\nu_{\min }$ the minimal normal expansion rate for the Hamilton flow of the principal symbol of $P_{0}$ at $\Gamma \subset{ }^{\mathrm{b}} T_{X}^{*} M$, as above; note that $\sigma$ is elliptic on $\Gamma$. This gives that for $\sigma \in \mathbb{R}, \widehat{P}_{0}(\sigma)-\widehat{P}_{0}(\sigma)^{*}$ is order $m-1$ in the large parameter pseudodifferential algebra, so, defining $z=\sigma /|\sigma|$, the semiclassical version gives

$$
\widetilde{P}_{0}-\widetilde{P}_{0}^{*} \in h \Psi_{\hbar}^{m-1}(X), z \in \mathbb{R}
$$

with

$$
\left.\sigma_{\hbar, m-1}\left(\frac{1}{2 i h}\left(\widetilde{P}_{0}-\widetilde{P}_{0}^{*}\right)\right)\right|_{\widetilde{\Gamma}}<\nu_{\min } / 2, z \in \mathbb{R},
$$

where $\widetilde{\Gamma}$ is the image of $\Gamma$ under the semiclassical identification. In particular, there is $\gamma_{\Gamma}>0$ and $\beta_{\Gamma}<\nu_{\min } / 2$ such that if $|\operatorname{Im} z|<h \gamma_{\Gamma}$ then

$$
\left.\sigma_{\hbar, m-1}\left(\frac{1}{2 i h}\left(\widetilde{P}_{0}-\widetilde{P}_{0}^{*}\right)\right)\right|_{\widetilde{\Gamma}}<\beta_{\Gamma}
$$

With this background, under our assumptions on the dynamics, propagating estimates from the radial points towards $H_{2}$, in particular through $\widetilde{\Gamma}$, and using the uniformity in parameters described above Theorem 4.7, we have:

Theorem 5.5. Let $C_{0}>0$. Suppose $P_{0}=P_{0}\left(v_{0}\right)$ satisfies (5.4) at $\Gamma, \widetilde{P}_{0}$ is the semiclassical rescaling of $\widehat{P}_{0}=\widehat{N}\left(P_{0}\right), s>1 / 2+\sup (\widetilde{\beta}) \gamma, s>1, \gamma<\gamma_{\Gamma}, \gamma_{\Gamma}>0$ as in (5.5). Then there is $h_{0}>0$ such that for $h<h_{0},|\operatorname{Im} z|<h \gamma$,

$$
\|u\|_{H_{h}^{s}} \lesssim h^{-2}\left\|\widetilde{P}_{0} u\right\|_{H_{h}^{s-m+1}}
$$

with the implied constant and $h_{0}$ uniform in $v_{0}$ with $\left|v_{0}\right| \leq C_{0}$.

Proof. This is immediate from piecing together the semiclassical propagation estimates from radial points (which is where $s>1 / 2+\sup (\widetilde{\beta}) \gamma$ is used, see the corresponding statement in the b-setting given in [33, Proposition 2.1, Footnote 20]) through $\widetilde{\Gamma}$, using Theorem 4.7, which is where $\gamma<\gamma_{\Gamma}$ is used and where $h^{-2}$, rather than $h^{-1}$, is obtained for the right hand side, to $H_{2} \cap X$, which is where $s>1$ is used.

An alternative proof would be using Dyatlov's setting [23] directly, together with the gluing of Datchev and Vasy [17], exactly as described in [51, Theorem 2.17].

Going back to the operator $P_{0}\left(v_{0}\right)$ satisfying the conditions stated at the beginning of this section, and under the additional assumption of uniform normal hyperbolicity as explained above, we can now obtain partial expansions of solutions to $P u=f$ at infinity, i.e. at $X$ :

Theorem 5.6. (Cf. [30, Theorem 7.9].) Let $0<\alpha<\min \left(1, \gamma_{\Gamma}\right)$. Suppose $P$ has a simple rank 1 resonance at 0 with resonant state 1 , and that all other resonances have imaginary part less than $-\alpha$. Let $\widetilde{s}>n / 2+6, s_{0}>\max (n / 2+1 / 2,1+$ $\sup (\widetilde{\beta}) \alpha)$, and assume $s_{0} \leq s \leq \widetilde{s}-4$. Let $0 \neq r \leq \alpha$. Then any solution $u \in H_{\mathrm{b}}^{s+4, r_{0}}(\Omega)^{\bullet},-$ of $P u=f$ with $f \in H_{\mathrm{b}}^{s+3, r}(\Omega)^{\bullet,-}$ satisfies $u \in \mathcal{X}^{s^{\prime}, r}$ with $s^{\prime}=s+4$ for $r<0$ and $s^{\prime}=s$ for $r>0$, and the following tame estimate holds:

$$
\|u\|_{\mathcal{X}^{s^{\prime}, r}} \lesssim\|f\|_{H_{\mathrm{b}}^{s+3, r}(\Omega) \bullet,-}+\|u\|_{H_{\mathrm{b}}^{s+4, r_{0}}(\Omega) \bullet,-}
$$




$$
+\left(\|f\|_{H_{\mathrm{b}}^{s_{0}, r}(\Omega) \bullet,-}+\|u\|_{H_{\mathrm{b}}^{s_{0}+1, r_{0}}(\Omega) \bullet-}\right)\|v\|_{\mathcal{X}^{\widetilde{s}, \alpha}} .
$$

Proof. The proof works in the same way as in the reference by an iterative argument that consists of rewriting $P u=f$ as $N(P) u=f-(P-N(P)) u$ and employing a contour deformation argument, see [51, Lemma 3.1] (which uses high-energy estimates for the inverse normal operator family $\widehat{P}(\sigma)^{-1}$ and the location of resonances, i.e. of the poles of this family), to improve on the decay of $u$ by $\alpha$ in each step, but losing an order of differentiability as we are treating $P-N(P)$ as an error term; using tame microlocal regularity for the equation $P u=f$, Corollary 5.4, one can regain this loss. We obtain $u \in H_{\mathrm{b}}^{s+4, r}$ after a finite number of iterations in case $r<0$, and $u \in H_{\mathrm{b}}^{s+4, r_{0}}$ for all $r_{0}<0$ in case $r>0$.

Assuming we are in the latter case, the next step of the iteration gives a partial expansion $u=c+u^{\prime}$ with $c \in \mathbb{C}$ (identified, as before, with $c \chi$, where $\chi$ is a smooth cutoff near the boundary) and $u^{\prime} \in H_{\mathrm{b}}^{s+2, r^{\prime}}$ for any $r^{\prime}$ satisfying $r^{\prime} \leq r$ and $r^{\prime}<\alpha$; here, we need $0<\alpha<\gamma_{\Gamma}$ so that the normally hyperbolic trapping estimate (5.6) holds with $\gamma>\alpha$, with loss of two derivatives. If $r=\alpha$, we can use this information to deduce

$$
N(P) u=f-(P-N(P)) u=f-\widetilde{f}, \quad \widetilde{f} \in H_{\mathrm{b}}^{\widetilde{s}, \alpha}+H_{\mathrm{b}}^{s, r^{\prime}+\alpha} \subset H_{\mathrm{b}}^{s, r},
$$

which implies that the expansion $u=c+u^{\prime}$ in fact holds with the membership $u^{\prime} \in H_{\mathrm{b}}^{s, r}$; notice the improvement in the weight. Therefore, $u \in \mathcal{X}^{s, r}$, finishing the proof.

Pipelining this result with the existence of solutions, Lemma 5.2, we therefore obtain:

Theorem 5.7. Under the assumptions of Theorem 5.6 with $r>0$ and $s>n / 2+2$, define the space

$$
\mathcal{Y}^{s, r}=\left\{u \in \mathcal{X}^{s, r}: P u \in H_{\mathrm{b}}^{s+3, r}(\Omega)^{\bullet,-}\right\} .
$$

Then the operator $P: \mathcal{Y}^{s, r} \rightarrow H_{\mathrm{b}}^{s+3, r}(\Omega)^{\bullet},-$ has a continuous inverse $S$ that satisfies the tame estimate

$$
\|S f\|_{\mathcal{X}^{s, r}} \leq C\left(s,\|v\|_{\mathcal{X}^{s_{0}, \alpha}}\right)\left(\|f\|_{H_{\mathrm{b}}^{s+3, r}(\Omega)^{\bullet,-}}+\|f\|_{H_{\mathrm{b}}^{s_{0}, r}(\Omega)^{\bullet,-}}\|v\|_{\mathcal{X}^{s+4, \alpha}}\right) .
$$

5.2. Solving quasilinear wave equations. We continue to work in the setting of the previous section. With the tame forward solution operator constructed in Theorem 5.7 in our hands, we are now in a position to use a Nash-Moser implicit function theorem to solve quasilinear wave equations. We use the following simple form of Nash-Moser, given in [46]:

Theorem 5.8. Let $\left(B^{s},|\cdot|_{s}\right)$ and $\left(\mathbf{B}^{s},\|\cdot\|_{s}\right)$ be Banach spaces for $s \geq 0$ with $B^{s} \subset$ $B^{t}$ and indeed $|v|_{t} \leq|v|_{s}$ for $s \geq t$, likewise for $\mathbf{B}^{*}$ and $\|\cdot\|_{*} ;$ put $B^{\infty}=\bigcap_{s} B^{s}$ and similarly $\mathbf{B}^{\infty}=\bigcap_{s} \mathbf{B}^{s}$. Assume there are smoothing operators $\left(S_{\theta}\right)_{\theta>1}: B^{\infty} \rightarrow B^{\infty}$ satisfying for every $v \in B^{\infty}, \theta>1$ and $s, t \geq 0$ :

$$
\begin{aligned}
\left|S_{\theta} v\right|_{s} & \leq C_{s, t} \theta^{s-t}|v|_{t} \text { if } s \geq t, \\
\left|v-S_{\theta} v\right|_{s} & \leq C_{s, t} \theta^{s-t}|v|_{t} \text { if } s \leq t .
\end{aligned}
$$


Let $\phi: B^{\infty} \rightarrow \mathbf{B}^{\infty}$ be a $C^{2}$ map, and assume that there exist $u_{0} \in B^{\infty}, d \in \mathbb{N}$, $\delta>0$ and constants $C_{1}, C_{2}$ and $\left(C_{s}\right)_{s \geq d}$ such that for any $u, v, w \in B^{\infty}$,

$$
\left|u-u_{0}\right|_{3 d}<\delta \Rightarrow\left\{\begin{array}{l}
\forall s \geq d, \quad\|\phi(u)\|_{s} \leq C_{s}\left(1+|u|_{s+d}\right) \\
\left\|\phi^{\prime}(u) v\right\|_{2 d} \leq C_{1}|v|_{3 d} \\
\left\|\phi^{\prime \prime}(u)(v, w)\right\|_{2 d} \leq C_{2}|v|_{3 d}|w|_{3 d} .
\end{array}\right.
$$

Moreover, assume that for every $u \in B^{\infty}$ with $\left|u-u_{0}\right|_{3 d}<\delta$ there exists an operator $\psi(u): \mathbf{B}^{\infty} \rightarrow B^{\infty}$ satisfying

$$
\phi^{\prime}(u) \psi(u) h=h
$$

and the tame estimate

$$
|\psi(u) h|_{s} \leq C_{s}\left(\|h\|_{s+d}+|u|_{s+d}\|h\|_{2 d}\right), \quad s \geq d,
$$

for all $h \in \mathbf{B}^{\infty}$. Then if $\left\|\phi\left(u_{0}\right)\right\|_{2 d}$ is sufficiently small depending on $\delta,\left|u_{0}\right|_{D}$ and $\left(C_{s}\right)_{s \leq D}$, where $D=16 d^{2}+43 d+24$, there exists $u \in B^{\infty}$ such that $\phi(u)=0$.

To apply this in our setting, we let $B^{s}=\mathcal{X}^{s, \alpha}(\Omega)=\mathbb{C} \oplus H_{\mathrm{b}}^{s, \alpha}(\Omega)^{\bullet,-}$ and $\mathbf{B}^{s}=$ $H_{\mathrm{b}}^{s, \alpha}(\Omega)^{\bullet},-$ with the corresponding norms; $\phi(u)$ will be the quasilinear equation, with implicit dependence on the forcing term. We now construct the smoothing operators $S_{\theta}$; we may assume, using a partition of unity, that $\Omega$ is the closure of an open subset of $\overline{\mathbb{R}_{+}^{n}}$, say $\Omega=\Omega(1)$, where we let $\Omega\left(x_{0}\right)=\left\{x \leq x_{0},|y| \leq 1\right\}$. Then there are bounded extension and restriction operators

$$
E: H_{\mathrm{b}}^{s, \alpha}(\Omega)^{\bullet,-} \rightarrow H_{\mathrm{b}}^{s, \alpha}\left(\overline{\mathbb{R}_{+}^{n}}\right), \quad R: H_{\mathrm{b}}^{s, \alpha}\left(\overline{\mathbb{R}_{+}^{n}}\right) \rightarrow H_{\mathrm{b}}^{s, \alpha}(\Omega)^{-,-},
$$

for $s \geq 0$; the operator $E$ can be constructed such that $\operatorname{supp} E v \subset\{x \leq 1\}$ for $v \in H_{\mathrm{b}}^{s, \alpha}(\Omega)^{\bullet,-}$. If we then define for $\theta>1$ and $v=(c, u) \in \mathcal{X}^{s, \alpha}$ :

$$
S_{\theta}^{1} v=\left(c, R S_{\theta}^{\prime} E v\right)
$$

where $S_{\theta}^{\prime}$ is a smoothing operator on $\overline{\mathbb{R}_{+}^{n}}$ with properties as in (5.8), then $S_{\theta}^{1}$ satisfies (5.8) in view of $R E$ being the identity on $H_{\mathrm{b}}^{s, \alpha}(\Omega)^{\bullet}$,- if the norms on the left hand side are understood to be $H_{\mathrm{b}}^{s, \alpha}\left(\overline{\mathbb{R}_{+}^{n}}\right)$-norms. However, note that $S_{\theta}^{1}$ does not map $\mathcal{X}^{\infty, \alpha}$ into itself, since smoothing operators such as $S_{\theta}^{\prime}$ enlarge supports; we will thus need to modify $S_{\theta}^{1}$ below to obtain the operators $S_{\theta}$. In order to construct $S_{\theta}^{\prime}$ on weighted b-Sobolev spaces $H_{\mathrm{b}}^{s, \alpha}$, it suffices by conjugation by the weight to construct it on the unweighted spaces $H_{\mathrm{b}}^{s}$; then, by a logarithmic change of coordinates, we only need to construct the smoothing operator $\widetilde{S}_{\theta}$ on the standard Sobolev spaces $H^{s}\left(\mathbb{R}^{n}\right)$, which we will do in Lemma 5.9 below. In order to deal with the issue of $S_{\theta}^{1}$ enlarging supports, we will define $\widetilde{S}_{\theta}$ such that

$$
v \in \mathcal{C}_{\mathrm{c}}^{\infty}\left(\mathbb{R}_{x^{\prime}, y^{\prime}}^{n}\right), \operatorname{supp} v \subset\left\{x^{\prime} \leq 0\right\} \Rightarrow \operatorname{supp} \widetilde{S}_{\theta} v \subset\left\{x^{\prime} \leq \theta^{-1 / 2}\right\} .
$$

In particular, when one undoes the logarithmic change of coordinates, this implies

$$
S_{\theta}^{1}: \mathcal{X}^{s, \alpha}(\Omega(1)) \rightarrow \mathcal{X}^{s, \alpha}\left(\Omega\left(\exp \left(\theta^{-1 / 2}\right)\right)\right)
$$

more generally, with $D_{\lambda}$ denoting dilations $D_{\lambda}(x, y)=(\lambda x, y)$ on $\overline{\mathbb{R}_{+}^{n}}$, we have

$$
S_{\theta}^{\lambda}:=\left(D_{\lambda}^{-1}\right)^{*} S_{\theta}^{1}\left(D_{\lambda}\right)^{*}: \mathcal{X}^{s, \alpha}(\Omega(\lambda)) \rightarrow \mathcal{X}^{s, \alpha}\left(\Omega\left(\lambda \exp \left(\theta^{-1 / 2}\right)\right)\right), \quad \lambda>0,
$$

with the operator norm independent of $\lambda$ near 1 . Now, in our application of Theorem 5.8, we will have

$$
\phi: \mathcal{X}^{\infty, \alpha}\left(\Omega\left(x_{0}\right)\right) \rightarrow H_{\mathrm{b}}^{\infty, \alpha}\left(\Omega\left(x_{0}\right)\right)^{\bullet,-} \text { for all } x_{0} \text { near } 1,
$$


and correspondingly we will have forward solution operators $\psi$ going in the reverse direction, with all relevant constants being uniform in $x_{0}$. Looking at the proof of Theorem 5.8 in [46], one only uses the smoothing operator $S_{\theta_{k}}$ with $\theta_{k}=\theta_{0}^{(5 / 4)^{k}}$ in the $k$-th step of the iteration, with $\theta_{0}$ chosen sufficiently large; in our situation, where we have (5.11), we can therefore use the smoothing operator

$$
S_{\theta_{k}}:=S_{\theta_{k}}^{\lambda_{k}}, \quad \lambda_{k}=\exp \left(\sum_{j=0}^{k-1} \theta_{j}^{-1 / 2}\right)
$$

in the $k$-th iteration step. Note that, for $\theta_{0}$ large, we have

$$
1=\lambda_{0} \leq \lambda_{1} \leq \cdots \leq \lambda_{\infty}=\exp \left(\sum_{j=0}^{\infty} \theta_{j}^{-1 / 2}\right) \leq 1+2 \theta_{0}^{-1 / 2}
$$

The solution $u$ to $\phi(u)=0$, obtained as a limit of an iterative scheme (see [46, Lemma 1]), therefore is an element of $\mathcal{X}^{s, \alpha}\left(\Omega\left(\lambda_{\infty}\right)\right)$. Taking the hyperbolic nature of the PDE $\phi(u)=0$ into account once more, it will then, in our concrete setting, be easy to conclude that in fact $u \in \mathcal{X}^{s, \alpha}(\Omega)$.

We now construct the smoothing operators on $\mathbb{R}^{n}$; the first step of the argument follows the Appendix of [46].

Lemma 5.9. There is a family $\left(\widetilde{S}_{\theta}\right)_{\theta>1}$ of operators on $H^{\infty}\left(\mathbb{R}^{n}\right)$ satisfying

$$
\begin{gathered}
\left\|\widetilde{S}_{\theta} v\right\|_{s} \leq C_{s, t} \theta^{s-t}\|v\|_{t} \text { if } s \geq t \geq 0, \\
\left\|v-\widetilde{S}_{\theta} v\right\|_{s} \leq C_{s, t} \theta^{s-t}\|v\|_{t} \text { if } 0 \leq s \leq t, \\
\operatorname{supp} \widetilde{S}_{\theta} v \subseteq\left\{x_{1} \leq \theta^{-1 / 2}\right\}
\end{gathered}
$$

for all $v \in H^{\infty}\left(\mathbb{R}^{n}\right)$ with $\operatorname{supp} v \subseteq H:=\left\{x_{1} \leq 0\right\}$. Here $\|\cdot\|_{s}$ denotes the $H^{s}\left(\mathbb{R}^{n}\right)$ norm, and we write $x=\left(x_{1}, x^{\prime}\right) \in \mathbb{R}^{n}$.

Proof. Choose $\chi=\chi_{1}\left(x_{1}\right) \chi_{2}\left(x^{\prime}\right) \in S\left(\mathbb{R}^{n}\right)$ with $\chi_{1} \in S(\mathbb{R}), \chi_{2} \in S\left(\mathbb{R}^{n-1}\right)$ so that the Fourier transform $\widehat{\chi}$ is identically 1 near 0 ; put $\chi_{\theta}(z)=\theta^{n} \chi(\theta z)$ and define the operator $C_{\theta} v=\chi_{\theta} * v$. Then $\left(C_{\theta} v\right)^{\widehat{ }}=\widehat{\chi_{\theta}} \widehat{v}$ with $\widehat{\chi_{\theta}}(\xi)=\widehat{\chi}(\xi / \theta)$, therefore (5.12) holds for $C_{\theta}$ in place of $\widetilde{S}_{\theta}$ with constants $C_{s, t}^{\prime}$ since $\widehat{\chi}$ decays super-polynomially, and (5.13) holds for $C_{\theta}$ in place of $\widetilde{S}_{\theta}$ with constants $C_{s, t}^{\prime}$ since $1-\widehat{\chi}(\xi)$ vanishes at $\xi=0$ with all derivatives.

Next, let $\psi \in \mathcal{C}^{\infty}\left(\mathbb{R}^{n}\right)$ be a smooth function depending only on $x_{1}$, i.e. $\psi=\psi\left(x_{1}\right)$, so that $\psi\left(x_{1}\right) \equiv 1$ for $x_{1} \in(-\infty, 1 / 2], \psi\left(x_{1}\right) \equiv 0$ for $x_{1} \in[1, \infty)$, and $0 \leq \psi \leq 1$. Put $\psi_{\theta}\left(x_{1}, x^{\prime}\right)=\psi\left(\theta x_{1}, x^{\prime}\right)$, and define

$$
\widetilde{S}_{\theta} v:=\psi_{\theta^{1 / 2}} C_{\theta} v \text {. }
$$

Condition (5.14) is satisfied by the support assumption on $\psi$. Let $\varphi=1-\psi$ and $\varphi_{\theta}=1-\psi_{\theta}$. To prove the other two conditions, we use the estimate

$$
\left\|\varphi_{\theta^{1 / 2}} C_{\theta} v\right\|_{s} \leq C_{s, N}^{\prime \prime} \theta^{-N}\|v\|_{L^{2}}, \quad \operatorname{supp} v \subset H, s, N \geq 0,
$$

which we will establish below. Taking this for granted, we obtain for $v$ with supp $v \subset$ $H$ :

$$
\left\|\widetilde{S}_{\theta} v\right\|_{s} \leq\left\|C_{\theta} v\right\|_{s}+\left\|\varphi_{\theta^{1 / 2}} C_{\theta} v\right\|_{s} \leq C_{s, t}^{\prime} \theta^{s-t}\|v\|_{t}+C_{s, 0}^{\prime \prime}\|v\|_{0}
$$

for $s \geq t \geq 0$, which is the estimate (5.12); and (5.13) follows from

$$
\left\|v-\widetilde{S}_{\theta} v\right\|_{s} \leq\left\|v-C_{\theta} v\right\|_{s}+\left\|\varphi_{\theta^{1 / 2}} C_{\theta} v\right\|_{s} \leq C_{s, t}^{\prime} \theta^{s-t}\|v\|_{t}+C_{s, t-s}^{\prime \prime} \theta^{s-t}\|v\|_{0}
$$


for $0 \leq s \leq t$.

We now prove (5.15) for $s \in \mathbb{N}_{0}$. For multiindices $\alpha=\left(\alpha_{1}, \alpha^{\prime}\right)$ with $|\alpha| \leq s$, we have for $v$ with $\operatorname{supp} v \subset H$ and for $\left(x_{1}, x^{\prime}\right) \in \operatorname{supp} \varphi_{\theta^{1 / 2}} C_{\theta} v$, which in particular implies $x_{1} \geq 1 /\left(2 \theta^{1 / 2}\right)$ :

$$
\begin{aligned}
& \partial^{\alpha}\left(\varphi_{\theta^{1 / 2}} C_{\theta} v\right)\left(x_{1}, x^{\prime}\right)=\sum_{j=0}^{\alpha_{1}}\left(\begin{array}{c}
\alpha_{1} \\
j
\end{array}\right) \theta^{\left(\alpha_{1}-j\right) / 2} \varphi^{\left(\alpha_{1}-j\right)}\left(\theta^{1 / 2} x_{1}\right) \\
& \quad \times \iint_{y_{1} \geq 1 /\left(2 \theta^{1 / 2}\right)} \theta^{n+j+\left|\alpha^{\prime}\right|} \chi_{1}^{(j)}\left(\theta y_{1}\right) \chi_{2}^{\left(\alpha^{\prime}\right)}\left(\theta y^{\prime}\right) v\left(x_{1}-y_{1}, x^{\prime}-y^{\prime}\right) d y_{1} d y^{\prime},
\end{aligned}
$$

thus

where

$$
\left\|\partial^{\alpha}\left(\varphi_{\theta^{1 / 2}} C_{\theta} v\right)\right\|_{L^{2}} \leq C_{s} \theta^{n+s}\left\|\check{\chi}_{\theta}\right\|_{L^{1}}\|v\|_{L^{2}}
$$

$$
\check{\chi}_{\theta}\left(x_{1}, x^{\prime}\right)= \begin{cases}0, & x_{1}<1 /\left(2 \theta^{1 / 2}\right), \\ \sum_{j=0}^{\alpha_{1}}\left|\chi_{1}^{(j)}\left(\theta x_{1}\right) \chi_{2}^{\left(\alpha^{\prime}\right)}\left(\theta x^{\prime}\right)\right| & \text { otherwise. }\end{cases}
$$

But $\left\|\check{\chi}_{\theta}\right\|_{L^{1}} \leq C_{N, s} \theta^{-N}$ for all $N$ : Indeed, this reduces to the statement that for a fixed $\chi_{0} \in S(\mathbb{R})$, one has

$$
\int_{1 /\left(2 \theta^{1 / 2}\right)}^{\infty}\left|\chi_{0}(\theta x)\right| d x \leq C_{N} \int_{\theta^{-1 / 2}}^{\infty}(\theta x)^{-2 N+1} d x=C_{N}^{\prime} \theta^{-N} .
$$

Hence, we obtain (5.15), and the proof is complete.

We now combine Theorem 5.7, giving the existence of tame forward solution operators, with Theorem 5.8, in the extended form described above, to solve quasilinear wave equations.

Theorem 5.10. Let $N \in \mathbb{N}$ and $c_{k} \in \mathcal{C}^{\infty}(\mathbb{C} ; \mathbb{R}), g_{k} \in\left(\mathcal{C}^{\infty}+H_{\mathrm{b}}^{\infty}\right)\left(M ; \mathrm{S}^{2} \mathrm{~b} T M\right)$ for $1 \leq k \leq N$; define the map $g: \mathcal{X}^{s, \alpha} \rightarrow\left(\mathcal{C}^{\infty}+H_{\mathrm{b}}^{s, \alpha}\right)\left(M ; \mathrm{S}^{2 \mathrm{~b}} T M\right)$ by $g(u)=$ $\sum_{k=1}^{N} c_{k}(u) g_{k}$ and assume that $\square_{g(0)}$ satisfies the assumptions of $\S 5.1$ and of Theorem 5.7; let us moreover assume that for small $u_{0}$, the metric $g\left(u_{0}\right)$ has uniform normally hyperbolic trapping in the sense described in $\S 4.4$. Further, let $N^{\prime} \in \mathbb{N}$ and define

$$
q\left(u,{ }^{\mathrm{b}} d u\right)=\sum_{j=1}^{N^{\prime}} u^{e_{j}} \prod_{k=1}^{N_{j}} X_{j k} u
$$

where

$$
e_{j}, N_{j} \in \mathbb{N}_{0}, N_{j} \geq 1, N_{j}+e_{j} \geq 2, X_{j k} \in\left(\mathcal{C}^{\infty}+H_{\mathrm{b}}^{\infty}\right) \mathcal{V}_{\mathrm{b}}
$$

Then there exists $C_{f}>0$ such that for all forcing terms $f \in H_{\mathrm{b}}^{\infty, \alpha}(\Omega)^{\bullet,-}$ satisfying $\|f\|_{H_{\mathrm{b}}^{\max (10, n+5), \alpha}(\Omega) \bullet,-} \leq C_{f}$, the equation

$$
\square_{g(u)} u=f+q\left(u,{ }^{\mathrm{b}} d u\right)
$$

has a unique solution $u \in \mathcal{X}^{\infty, \alpha}$.

If more generally $g\left(u,{ }^{\mathrm{b}} d u\right)=\sum_{k=1}^{N} c_{k}\left(u, X_{1} u, \ldots, X_{L} u\right) g_{k}$, where $X_{1}, \ldots, X_{L} \in$ $\mathcal{V}_{\mathrm{b}}(M)$ and $c_{k} \in \mathcal{C}^{\infty}\left(\mathbb{C}^{1+L} ; \mathbb{R}\right)$, with $g\left(u_{0}, 0\right)$ uniformly normally hyperbolic for small $u_{0}$, then there exists $C_{f}>0$ such that for all forcing terms $f \in H_{\mathrm{b}}^{\infty, \alpha}(\Omega)^{\bullet,-}$ satisfying $\|f\|_{H_{\mathrm{b}}^{\max (12, n+5), \alpha}(\Omega) \bullet,-} \leq C_{f}$, the equation

$$
\square_{g(u, \mathrm{~b} d u)} u=f+q\left(u,{ }^{\mathrm{b}} d u\right)
$$

has a unique solution $u \in \mathcal{X}^{\infty, \alpha}$. 
In both cases, if one instead assumes that the trapping for $g(0,0)$ is r-normally hyperbolic for every $r$, then for every fixed $s \in \mathbb{R}$, one has solvability with $u \in \mathcal{X}^{s, \alpha}$ provided the norm bound $C_{f}=C_{f}(s)>0$ on $f$ (on the same spaces as before) is sufficiently small.

We point out that if all vector fields, coefficients and data are real, then $u$ is real-valued as well.

Proof of Theorem 5.10. We write $|\cdot|_{s}$ for the $\mathcal{X}^{s, \alpha}$-norm and $\|\cdot\|_{s}$ for the $H_{\mathrm{b}}^{s, \alpha}$ norm. (For brevity, we do not specify the underlying set, which, in the notation of $\S 5.1$, is $\mathfrak{t}_{1}^{-1}([-\lambda, \infty)) \cap \mathfrak{t}_{2}^{-1}([0, \infty))$ for varying $\lambda \geq 0$.) We define the map

$$
\phi(u ; f)=\square_{g(u)} u-q\left(u,{ }^{\mathrm{b}} d u\right)-f
$$

and check that it satisfies the conditions of Theorem 5.8 with $u_{0}=0$. From the definition of $\square_{g(u)}$ and the tame estimates for products, reciprocals and compositions, Corollary 3.2 and Propositions 3.4 and 3.7, we obtain

$$
\|\phi(u ; f)\|_{s} \leq\|f\|_{s}+C\left(|u|_{s_{0}+2}\right)\left(1+|u|_{s+2}\right), \quad s \geq s_{0}>n / 2+1,
$$

thus the first estimate of (5.9) for $3 d \geq s_{0}+2, d \geq s_{0}, d \geq 2$. Next, we have $\phi^{\prime}(u ; f) v=\left(\square_{g(u)}+L\left(u,{ }^{\mathrm{b}} d u\right)\right) v$, where the first order b-differential operator $L$ is of the form

$$
L=\sum_{|\beta| \leq 1}\left(\sum_{1 \leq|\alpha| \leq 2} a_{\alpha \beta}\left(u,{ }^{\mathrm{b}} d u\right)^{\mathrm{b}} D^{\alpha} u\right){ }^{\mathrm{b}} D^{\beta}+\sum_{|\beta|=1} a_{\beta}\left(u,{ }^{\mathrm{b}} d u\right) u^{\mathrm{b}} D^{\beta},
$$

with the second sum capturing one term of the linearization of terms $u^{e_{j}} X_{j 1} u$ in $q$ (i.e. terms for which $N_{j}=1$ ). In particular,

$$
\phi^{\prime}(u ; f)=P_{0}\left(u_{0}\right)+\widetilde{P}\left(u,{ }^{\mathrm{b}} D u,{ }^{\mathrm{b}} D^{2} u\right),
$$

where $P_{0} \in \operatorname{Diff}_{\mathrm{b}}^{2}$ and $\widetilde{P} \in H_{\mathrm{b}}^{s-1, \alpha} \operatorname{Diff}_{\mathrm{b}}^{2}+H_{\mathrm{b}}^{s-2, \alpha} \operatorname{Diff}_{\mathrm{b}}^{1}$ for $u \in \mathcal{X}^{s, \alpha}$; in fact, the leading order coefficient has regularity $H_{\mathrm{b}}^{s, \alpha}$, but we give up one derivative for direct comparison with (5.1). Therefore,

$$
\left\|\phi^{\prime}(u ; f) v\right\|_{s} \leq C\left(|u|_{s+2}\right)|v|_{s+2}, \quad s>n / 2+1,
$$

which gives the second estimate of (5.9) for $2 d>n / 2+1$ and $3 d \geq 2 d+2$. Next, we observe that $\phi^{\prime \prime}(u ; f)(v, w)$ is bilinear in $v, w$, involves up to two b-derivatives of each $v$ and $w$, and the coefficients depend on up to two b-derivatives of $u$, thus

$$
\left\|\phi^{\prime \prime}(u ; f)(v, w)\right\|_{s} \leq C\left(|u|_{s+2}\right)|v|_{s+2}|w|_{s+2}, \quad s>n / 2+1,
$$

which gives the third estimate of (5.9) for $3 d>n / 2+3,3 d \geq 2 d+2$. In summary, we obtain (5.9) for integer $d>n / 2+1$.

Finally, we determine $d$ so that we have the tame estimate (5.10): Given $u \in$ $\mathcal{X}^{s+5, \alpha}$, we can write $\phi^{\prime}(u ; f)$ as in $(5.20)$, with $P_{0} \in \operatorname{Diff}_{\mathrm{b}}^{2}$ and $\widetilde{P} \in H_{\mathrm{b}}^{s+4, \alpha} \operatorname{Diff}_{\mathrm{b}}^{2}+$ $H_{\mathrm{b}}^{s+3, \alpha} \operatorname{Diff}_{\mathrm{b}}^{1}$; hence, by Theorem 5.7 , we obtain a solution operator

$$
\begin{gathered}
\psi(u ; f): H_{\mathrm{b}}^{s+3, \alpha} \rightarrow \mathcal{X}^{s, \alpha}, \\
|\psi(u ; f) v|_{s} \leq C\left(s,|u|_{s_{0}}\right)\left(\|v\|_{s+3}+\|v\|_{s_{0}}|u|_{s+5}\right),
\end{gathered}
$$

where $s, s_{0}>n / 2+2$, provided $|u|_{s_{0}}$ is small enough so that all dynamical and geometric hypotheses hold for $\phi^{\prime}(u ; f)$. Notice that the subprincipal term of $\phi^{\prime}(u ; f)$ can differ from that of $\square_{g(0)}$ by terms of the form $a\left(u_{0}\right) u_{0}{ }^{\mathrm{b}} D^{\beta}, a \in \mathcal{C}^{\infty},|\beta|=1$, see (5.19); however, since such terms eliminate constants, the simple rank 1 resonance 
at 0 with resonant state 1 does not change; and moreover such terms are small because of the factor $u_{0}$, hence high energy estimates still hold in a (possibly slightly smaller) strip in the analytic continuation, see the remark below [23, Theorem 1]. Since $s_{0}$ is independent of $s$, we have (5.21) for all $s>n / 2+2$, in particular $\psi(u ; f): H_{\mathrm{b}}^{\infty, \alpha} \rightarrow \mathcal{X}^{\infty, \alpha}$. Now, (5.21) implies that (5.10) holds for $d>n / 2+2$, $d \geq 5$, so we need to control $\max (10, n+5)$ derivatives of $f$.

Thus, we can apply Nash-Moser iteration, Theorem 5.8, to obtain a solution $u \in \mathcal{X}^{s, \alpha}$ of the $\operatorname{PDE}$ (5.17), with the caveat that $u$ is a priori supported on a space slightly larger than $\Omega$, in the sense that $\operatorname{supp} u$ might not be contained in the future of $H_{1}$. (The support generally extends past $H_{2}$, but this does not concern us since we are always restricting ourselves to the region bounded by the connected components of $\mathrm{H}_{2}$.) However, local uniqueness for quasilinear hyperbolic equations, see e.g. [49, $\S 16.3]$, implies that $u$ in fact vanishes in the past of $H_{1}$, hence is supported in $\Omega$, and that $u$ is the unique solution of (5.17), finishing the proof of the first part.

The proof of the second part proceeds analogously; now the operator $L$ in (5.19) involves an additional term

$$
\sum_{|\beta|=2}\left(\sum_{|\alpha|=1} a_{\alpha \beta}^{\prime}\left(u,{ }^{\mathrm{b}} d u\right)^{\mathrm{b}} D^{\alpha} u\right){ }^{\mathrm{b}} D^{\beta}
$$

since the first order terms of the expression of $\square_{g(u, b d u)}$ involve derivatives of the metric, hence second derivatives of $u$; notice however that the additional term can be put into $\widetilde{P}$ due to the factor of $d u$. Therefore, if now $u \in \mathcal{X}^{s+6, \alpha}$, then $\widetilde{P}$ in (5.20) lies in the space $H_{\mathrm{b}}^{s+4, \alpha} \operatorname{Diff}_{\mathrm{b}}^{2} \subset H_{\mathrm{b}}^{s+4, \alpha} \operatorname{Diff}_{\mathrm{b}}^{2}+H_{\mathrm{b}}^{s+3, \alpha} \operatorname{Diff}_{\mathrm{b}}^{1}$; the rest of the argument then goes through, the only change being that the $|u|_{s+5}$ norm in (5.21) needs to be replaced by $|u|_{s+6}$, hence the new requirement $d \geq 6$. Therefore, we need to control $\max (12, n+5)$ derivatives of $f$ for the second part.

Lastly, we recall that $r$-normal hyperbolicity for any finite $r$ is structurally stable, hence for any fixed $r \in \mathbb{R}$, the metric $g\left(u_{0}, 0\right)$ has normally hyperbolic trapping for sufficiently small $u_{0}$, with stable/unstable manifolds and trapped sets of class $\mathcal{C}^{r}$, depending continuously on $u_{0}$. Since the estimates at normally hyperbolic trapping on any fixed Sobolev space only require a finite number of regularity (since they then rely on the finiteness of only finitely many norms and seminorms of the manifolds and operators involved) and since Nash-Moser iteration only requires a finite number of derivatives if one is merely interested in obtaining a solution with finite b-regularity (see also Remark 5.12 below), the above arguments imply solvability in $\mathcal{X}^{s, \alpha}$ provided the norm of $f$ in the stated spaces is small enough, depending on $s$ : Indeed, if the norm of $f$ is $\leq C_{f}$, then the norm of every iterate $u_{k}$ in the Nash-Moser scheme will be bounded by a constant multiple of $C_{f}$ in a suitable (fixed) Sobolev space, hence the corresponding metrics $g\left(u_{k},{ }^{\mathrm{b}} d u_{k}\right)$, restricted to the boundary at future infinity $\Omega \cap \partial M$, will be close to $g(0,0)$ and thus have $r$-normally hyperbolic trapping, where $r$ can be any fixed arbitrarily large real number if $C_{f}>0$ is sufficiently small, depending on $r$. Therefore, given $s$, we merely need to pick $C_{f}>0$ so small that the corresponding regularity $\mathcal{C}^{r}$ of the trapping is sufficient for all estimates at the trapping to hold on all (finite regularity!) Sobolev spaces which appear (explicitly and implicitly) in the above proof. 
Remark 5.11. In the asymptotically de Sitter setting considered in [30], the above Theorem extends [30, Theorem 8.8] (at the cost of requiring the control of more derivatives) since we allow the dependence of the metric $g\left(u,{ }^{\mathrm{b}} d u\right)$ on ${ }^{\mathrm{b}} d u$ as well.

Remark 5.12. An inspection of the proof of the abstract Nash-Moser theorem 5.8 in [46] shows that there are constants $C$ and $s_{0}$, depending only on the 'loss of derivatives' $d$, such that the following holds: In order to obtain a solution $u \in \mathcal{X}^{s, \alpha}$ for some finite $s \geq s_{0}$, it is sufficient to take $f \in H_{\mathrm{b}}^{C s, \alpha}$, still assuming the norm of $f$ in the space indicated in the statement of Theorem 5.10 to be small.

Theorem 5.10 immediately implies the following result on Kerr-de Sitter space:

Corollary 5.13. Under the assumptions of Theorem 5.10, the quasilinear wave equation (5.17), resp. (5.18), on a 4-dimensional asymptotically Kerr-de Sitter space with $|a| \ll M_{\bullet}$ has a unique global solution in the space $\mathcal{X}^{s, \alpha}$ if the $H_{\mathrm{b}}^{10, \alpha}(\Omega)^{\bullet--}$ norm, resp. $H_{\mathrm{b}}^{12, \alpha}(\Omega)^{\bullet,-}$-norm, of the forcing term $f \in H_{\mathrm{b}}^{\infty, \alpha}(\Omega)^{\bullet,-}$ is sufficiently small.

Proof. For a verification of the dynamical assumptions for asymptotically Kerr-de Sitter spaces, we refer the reader to $[51, \S 6]$; the resonances on the other hand were computed by Dyatlov [21], and also by the authors using a perturbation argument [31].

5.3. Solving quasilinear Klein-Gordon equations. The only difference between wave and Klein-Gordon equations with mass $m$ (which is to be distinguished from the black hole mass $M_{\bullet}$ ) is that the resonance of the Klein-Gordon operator $\square-m^{2}$ with largest imaginary part, which gives the leading order asymptotics, is no longer at 0 for $m \neq 0$. Thus, if $\sigma_{1}, \sigma_{2} \in \mathbb{C}$ are the first two resonances of $\square-m^{2}$, i.e. there are no further resonances $\sigma$ with $\operatorname{Im} \sigma \geq \operatorname{Im} \sigma_{2}$, assume

$$
0<-\operatorname{Im} \sigma_{1}<r<-\operatorname{Im} \sigma_{2}
$$

and assume moreover that the high energy estimates for the normal operator family of $\square-m^{2}$ hold in $\operatorname{Im} \sigma \geq-r$, the only change in the statement of Theorem 5.6 for Klein-Gordon operators is that the conclusion now is $u \in \mathcal{X}_{\sigma_{1}}^{s, r}$, where $\mathcal{X}_{\sigma_{1}}^{s, r}=$ $\mathbb{C} \oplus H_{\mathrm{b}}^{s, r}(\Omega)^{\bullet},-$, with $\left(c, u^{\prime}\right)$ identified with $c x^{i \sigma_{1}} \chi+u^{\prime}$ for a smooth cutoff $\chi$ near the boundary.

Remark 5.14. There are more cases of potential interest: If $r<-\operatorname{Im} \sigma_{1}$, we obtain $u \in H_{\mathrm{b}}^{s, r}(\Omega)^{\bullet,-}$; if $r<0$, the statement of Theorem 5.6 is unchanged; and if $\operatorname{Im} \sigma_{1}$ and $\operatorname{Im} \sigma_{2}$ are close enough together (including the case that $\sigma_{1}$ is a double resonance) and there is a spectral gap below $\sigma_{2}$, one gets two terms in the expansion of $u$. For brevity, we only explain one scenario here. See also the related discussion in $[30, \S 8.4]$.

We thus obtain the following adapted version of Theorem 5.7:

Theorem 5.15. In the notation of $\$ 5.2$, under the above assumptions and for $s>n / 2+2$, define the space

$$
\mathcal{Y}_{\sigma_{1}}^{s, r}=\left\{u \in \mathcal{X}_{\sigma_{1}}^{s, r}: P u \in H_{\mathrm{b}}^{s+3, r}(\Omega)^{\bullet,-}\right\} .
$$

Then the operator $P: \mathcal{Y}^{s, r} \rightarrow H_{\mathrm{b}}^{s+3, r}(\Omega)^{\bullet,-}$ has a continuous inverse $S$ that satisfies the tame estimate

$$
\|S f\|_{\mathcal{X}_{\sigma_{1}}^{s, r}} \leq C\left(s,\|v\|_{\mathcal{X}_{\sigma_{1}}^{s_{0}, \alpha}}\right)\left(\|f\|_{H_{\mathrm{b}}^{s+3, r}(\Omega) \bullet,-}+\|f\|_{H_{\mathrm{b}}^{s_{0}, r}(\Omega) \bullet,-}\|v\|_{\mathcal{X}_{\sigma_{1}}^{s+4, \alpha}}\right) .
$$


This immediately gives:

Theorem 5.16. Under the above assumptions and the assumption $\alpha<-2 \operatorname{Im} \sigma_{1}$, let $N, N^{\prime} \in \mathbb{N}$ and $c_{k} \in \mathcal{C}^{\infty}(\mathbb{C} ; \mathbb{R}), g_{k} \in\left(\mathcal{C}^{\infty}+H_{\mathrm{b}}^{\infty}\right)\left(M ; \mathrm{S}^{2} \mathrm{~b} T M\right)$ for $1 \leq k \leq N$; define the map $g: \mathcal{X}_{\sigma_{1}}^{s, \alpha} \rightarrow\left(\mathcal{C}^{\infty}+H_{\mathrm{b}}^{s, \alpha}\right)\left(M ; \mathrm{S}^{2} \mathrm{~b} T M\right)$ by $g(u)=\sum_{k=1}^{N} c_{k}(u) g_{k}$ and assume that $\square_{g(0)}$ satisfies the assumptions of $\S 5.1$ and of Theorem 5.15. Moreover, define

$$
q\left(u,{ }^{\mathrm{b}} d u\right)=\sum_{j=1}^{N^{\prime}} a_{j} u^{e_{j}} \prod_{k=1}^{N_{j}} X_{j k} u
$$

where

$$
e_{j}, N_{j} \in \mathbb{N}_{0}, e_{j}+N_{j} \geq 2, a_{j} \in \mathcal{C}^{\infty}, X_{j k} \in\left(\mathcal{C}^{\infty}+H_{\mathrm{b}}^{\infty}\right) \mathcal{V}_{\mathrm{b}}
$$

Then there exists $C_{f}>0$ such that for all forcing terms $f \in H_{\mathrm{b}}^{\infty, \alpha}(\Omega)^{\bullet,-}$ satisfying $\|f\|_{H_{\mathrm{b}}^{\max (10, n+5), \alpha}(\Omega) \bullet,-} \leq C_{f}$, the equation

$$
\left(\square_{g(u)}-m^{2}\right) u=f+q\left(u,{ }^{\mathrm{b}} d u\right)
$$

has a unique solution $u \in \mathcal{X}_{\sigma_{1}}^{\infty, \alpha}$.

If more generally $g\left(u,{ }^{\mathrm{b}} d u\right)=\sum_{k=1}^{N} c_{k}\left(u, X_{1} u, \ldots, X_{L} u\right)$, where $X_{1}, \ldots, X_{L} \in$ $\mathcal{V}_{\mathrm{b}}(M)$ and $c_{k} \in \mathcal{C}^{\infty}\left(\mathbb{C}^{1+L} ; \mathbb{R}\right)$, then there exists $C_{f}>0$ such that for all forcing terms $f \in H_{\mathrm{b}}^{\infty, \alpha}(\Omega)^{\bullet,-}$ satisfying $\|f\|_{H_{\mathrm{b}}^{\max (12, n+5), \alpha}(\Omega)^{\bullet,-}} \leq C_{f}$, the equation

$$
\left(\square_{g(u, \mathrm{~b} d u)}-m^{2}\right) u=f+q\left(u,{ }^{\mathrm{b}} d u\right)
$$

has a unique solution $u \in \mathcal{X}^{\infty, \alpha}$.

Together with Theorem 5.10, this proves Theorem 2.

Proof of Theorem 5.16. The proof proceeds as the proof of Theorem 5.10. Notice that we allow the nonlinear term $q$ to be more general, the point being that firstly, any at least quadratic expression in $\left(u,{ }^{\mathrm{b}} d u\right)$ with $u \in \mathcal{X}_{\sigma_{1}}^{s, \alpha}$ gives an element of $H_{\mathrm{b}}^{s, \alpha}$, and secondly, every element in $\mathcal{X}_{\sigma_{1}}^{s, \alpha}$ vanishes at the boundary, thus the normal operator family of the linearization of $\square_{g(u)}-m^{2}-q\left(u,{ }^{\mathrm{b}} d u\right)-f$ at any $u \in \mathcal{X}_{\sigma_{1}}^{s, \alpha}$ is equal to the normal operator family of $\square_{g(0)}-m^{2}$, for which one has high energy estimates by assumption.

We point out that the trapping trivially is uniformly normally hyperbolic since the normal operator family $\widehat{N}\left(\square_{g\left(u,{ }^{b} d u\right)}\right)$ is in fact independent of $u \in \mathcal{X}_{\sigma_{1}}^{s, \alpha}$; hence one indeed obtains a solution in $\mathcal{X}_{\sigma_{1}}^{\infty, \alpha}$.

By [33, Lemma 3.5], the assumptions of Theorem 5.16 are satisfied on asymptotically Kerr-de Sitter spaces as long as the mass parameter $m$ is small:

Corollary 5.17. Under the assumptions of Theorem 5.16 and for a and $m>0$ sufficiently small, the quasilinear Klein-Gordon equation (5.23), resp. (5.24), on a 4-dimensional asymptotically Kerr-de Sitter space with angular momentum a has a unique global smooth (i.e. conormal, in the space $\mathcal{X}_{\sigma_{1}}^{\infty, \alpha}$ ) solution if the $H_{\mathrm{b}}^{10, \alpha}(\Omega)^{\bullet,-}$ norm, resp. $H_{\mathrm{b}}^{12, \alpha}(\Omega)^{\bullet},-$-norm, of the forcing term $f \in H_{\mathrm{b}}^{\infty, \alpha}(\Omega)^{\bullet,-}$ is sufficiently small. 
5.4. Proofs of Theorems 3 and 4. Finally, following the same arguments as used in the previous section, we indicate how to prove the general theorems stated in the introduction. We continue to use, but need to generalize the setting considered in §5.1: Namely, generalizing (5.1), we now allow $L$ to be any first order b-differential operator, and correspondingly need information on the skew-adjoint part of $P_{0}$; concretely, we define $\widehat{\beta}$ at the (generalized) radial sets $L_{ \pm}$, using the same notation as in (5.2), by

$$
\left.\sigma_{\mathrm{b}, 1}\left(\frac{1}{2 i}\left(P_{0}-P_{0}^{*}\right)\right)\right|_{L_{ \pm}}= \pm \widehat{\beta} \beta_{0} \rho
$$

Moreover, at the trapped set $\Gamma=\Gamma^{-} \cup \Gamma^{+}$, we assume that

$$
\left.\mathrm{e}_{1}\right|_{\Gamma}<\nu_{\min } / 2, \quad \mathrm{e}_{1}=|\sigma|^{-1} \sigma_{\mathrm{b}, 1}\left(\frac{1}{2 i}\left(P_{0}-P_{0}^{*}\right)\right),
$$

with $\nu_{\min }$ the minimal normal expansion rate for the Hamilton flow of the principal symbol of $P_{0}$, and $\sigma$ the Mellin dual variable of $x$ after an identification of a collar neighborhood of $X$ in $M$ with $\left[0, \epsilon^{\prime}\right)_{x} \times X$; note that $\sigma$ is elliptic on $\Gamma$. Let $r_{\text {th }}$ be the threshold weight for the first part of Theorem 4.5 , i.e. $r_{\mathrm{th}}=-\sup \mathrm{e}_{1} / c_{\partial}$ with $c_{\partial}$ as defined in (4.15).

Then Corollary 5.4 holds in the current, more general setting, provided we assume $r<r_{\text {th }}$ and $s^{\prime}>1+\sup _{L_{ \pm}}(r \widetilde{\beta}-\widehat{\beta})$. Likewise, we obtain the high energy estimates of Theorem 4.7 under the assumption $s>1 / 2+\sup _{L_{ \pm}}(\gamma \widetilde{\beta}-\widehat{\beta})$.

In order to generalize Theorem 5.6, we first choose $0<r_{+}<1$ such that

$$
\left.\left(\mathrm{e}_{1}+r_{+} c_{\partial}\right)\right|_{\Gamma}<\nu_{\min } / 2,
$$

which holds for sufficiently small $r_{+}$in view of (5.26) by the compactness of $\Gamma$ in ${ }^{\mathrm{b}} S^{*} M$. We moreover assume that there are no (nonzero) resonances in $\operatorname{Im} \sigma>-r_{+}$ in the case of Theorem 3 (Theorem 4), and we assume further that $0<\alpha<r_{+}$. Then in the proof of Theorem 5.6, ignoring the issue of threshold regularities at radial sets momentarily, we can use the contour shifting argument without loss of derivatives up to, but excluding, the weight $r_{\text {th }}$, corresponding to the contour of integration $\operatorname{Im} \sigma=-r_{\mathrm{th}}$. Shifting the contour further down, we cannot use the non-smooth real principal type estimate at $\Gamma$ anymore and thus lose 2 derivatives at each step; the total number of additional steps needed to shift the contour down to $\operatorname{Im} \sigma=-\alpha$ is easily seen to be at most

$$
N=\max \left(0,\left\lceil\frac{\alpha-r_{\mathrm{th}}}{\alpha}\right\rceil+1\right)
$$

hence in order to have the final conclusion that $u$ has an expansion with remainder in $H_{\mathrm{b}}^{s, \alpha}$, we need to assume that $u$ initially is known to have regularity $H_{\mathrm{b}}^{s+2 N, r_{0}}$ for any $r_{0} \in \mathbb{R}$, which in turn requires $\widetilde{s} \geq s+2 N$ and $f \in H_{\mathrm{b}}^{s+2 N-1, r_{0}}$ for the first, lossless, part of the argument to work. Taking the regularity requirements at the radial sets into account, we further need to assume $s \geq s_{0}>\max (n+1 / 2,1+$ $\sup (r \widetilde{\beta}-\widehat{\beta}))$. Under these assumptions, the proof of Theorem 5.6 applies, mutatis mutandis, to our current situation, and we obtain a tame solution operator as in Theorem 5.7, which now loses $2 N-1$ derivatives.

Thus, we can prove Theorems 3 and 4 using the same arguments which we used in the proof of Theorem 5.10; the 'loss of derivatives' parameter $d$ now needs to satisfy the conditions

$$
d \geq 2 N+3, d>n / 2+6, d>1+\sup (r \widetilde{\beta}-\widehat{\beta}),
$$


with the first condition being the actual loss of derivatives, the second one coming from $s>n / 2+6$ certainly being a high enough regularity for $\widetilde{s}=s+2 N$ to be $>n / 2+6$, which is required for the application of the non-smooth microlocal regularity results, and the last condition being the threshold regularity (for the non-smooth estimates) at the radial sets.

Remark 5.18. We again point out that these theorems hold under general hypotheses, as explained in $\S 2$; one merely needs to use the tame estimates on manifolds, which hold by the discussion in Remarks 4.4 and 4.6.

\section{REFERENCES}

[1] Lars Andersson and Pieter Blue. Uniform energy bound and asymptotics for the Maxwell field on a slowly rotating Kerr black hole exterior, Preprint, arXiv:1310.2664, 2013.

[2] Alain Bachelot. Gravitational scattering of electromagnetic field by Schwarzschild black-hole. Ann. Inst. H. Poincaré Phys. Théor., 54(3):261-320, 1991.

[3] Alain Bachelot. Scattering of electromagnetic field by de Sitter-Schwarzschild black hole. In Nonlinear hyperbolic equations and field theory (Lake Como, 1990), Pitman Res. Notes Math. Ser., Longman Sci. Tech., Harlow, 253:23-35, 1992.

[4] Michael Beals and Michael Reed. Microlocal regularity theorems for nonsmooth pseudodifferential operators and applications to nonlinear problems. Trans. Amer. Math. Soc., 285(1):159-184, 1984.

[5] Pieter Blue and Avy Soffer. Phase space analysis on some black hole manifolds. J. Funct. Anal., 256(1):1-90, 2009.

[6] Jean-François Bony and Dietrich Häfner. Decay and non-decay of the local energy for the wave equation on the de Sitter-Schwarzschild metric. Comm. Math. Phys., 282(3):697-719, 2008.

[7] Brandon Carter. Global structure of the Kerr family of gravitational fields. Phys. Rev., 174:1559-1571, 1968.

[8] Brandon Carter. Hamilton-Jacobi and Schrödinger separable solutions of Einstein's equations. Comm. Math. Phys., 10:280-310, 1968.

[9] Mihalis Dafermos, Gustav Holzegel and Igor Rodnianski. A scattering theory construction of dynamical vacuum black holes. Preprint, arxiv:1306.5364, 2013.

[10] Mihalis Dafermos and Igor Rodnianski. The black hole stability problem for linear scalar perturbations. In T. Damour et al, editor, Proceedings of the Twelfth Marcel Grossmann Meeting on General Relativity, pages 132-189. World Scientific, Singapore, 2011. arXiv:1010.5137.

[11] Mihalis Dafermos and Igor Rodnianski. Decay of solutions of the wave equation on Kerr exterior space-times I-II: The cases of $|a| \ll m$ or axisymmetry. Preprint, arXiv:1010.5132, 2010.

[12] Mihalis Dafermos and Igor Rodnianski. A proof of Price's law for the collapse of a selfgravitating scalar field. Invent. Math., 162(2):381-457, 2005.

[13] Mihalis Dafermos and Igor Rodnianski. The red-shift effect and radiation decay on black hole spacetimes. Comm. Pure Appl. Math, 62:859-919, 2009.

[14] Mihalis Dafermos and Igor Rodnianski. The wave equation on Schwarzschild-de Sitter space times. Preprint, arXiv:07092766, 2007.

[15] Mihalis Dafermos and Igor Rodnianski. Lectures on black holes and linear waves. CMI/AMS Publications, 2013.

[16] Mihalis Dafermos, Igor Rodnianski, and Yakov Shlapentokh-Rothman. Decay for solutions of the wave equation on Kerr exterior spacetimes III: The full subextremal case $|a|<m$. Preprint, arXiv:1402.7034, 2014.

[17] Kiril Datchev and András Vasy. Gluing semiclassical resolvent estimates via propagation of singularities. International Mathematics Research Notices, 2012(23):5409-5443, 2012.

[18] Roland Donninger, Wilhelm Schlag, and Avy Soffer. A proof of Price's law on Schwarzschild black hole manifolds for all angular momenta. Adv. Math., 226(1):484-540, 2011.

[19] Semyon Dyatlov. Asymptotics of linear waves and resonances with applications to black holes. Preprint, arXiv:1305.1723, 2013.

[20] Semyon Dyatlov. Exponential energy decay for Kerr-de Sitter black holes beyond event horizons. Math. Res. Lett., 18(5):1023-1035, 2011. 
[21] Semyon Dyatlov. Quasi-normal modes and exponential energy decay for the Kerr-de Sitter black hole. Comm. Math. Phys., 306(1):119-163, 2011.

[22] Semyon Dyatlov. Resonance projectors and asymptotics for r-normally hyperbolic trapped sets. Preprint, arXiv:1301.5633, 2013.

[23] Semyon Dyatlov. Spectral gaps for normally hyperbolic trapping. Preprint, arXiv:1403.6401, 2014.

[24] Semyon Dyatlov and Maciej Zworski. Trapping of waves and null geodesics for rotating black holes. Phys. Rev. D, 88, 084037, 2013.

[25] Felix Finster, Niky Kamran, Joel Smoller, and Shing-Tung Yau. Decay of solutions of the wave equation in the Kerr geometry. Comm. Math. Phys., 264(2):465-503, 2006.

[26] Felix Finster, Niky Kamran, Joel Smoller, and Shing-Tung Yau. Linear waves in the Kerr geometry: a mathematical voyage to black hole physics. Bull. Amer. Math. Soc. (N.S.), 46(4):635-659, 2009.

[27] Christian Gérard and Johannes Sjöstrand. Resonances en limite semiclassique et exposants de Lyapunov. Comm. Math. Phys., 116(2):193-213, 1988.

[28] Richard S. Hamilton. The inverse function theorem of Nash and Moser. Bull. Amer. Math. Soc. (N.S.), 7(1):65-222, 1982.

[29] Peter Hintz. Resonance expansions for tensor-valued waves on Kerr-de Sitter space. Preprint, arXiv:1502.03183, 2015

[30] Peter Hintz. Global well-posedness of quasilinear wave equations on asymptotically de Sitter spaces. Preprint, arXiv:1311.6859, 2013.

[31] Peter Hintz and András Vasy. Asymptotics for the wave equation on differential forms on Kerr-de Sitter space. Preprint, arXiv:1502.03179, 2015.

[32] Peter Hintz and András Vasy. Non-trapping estimates near normally hyperbolic trapping. Math. Res. Lett., 21(6):1277-1304, 2014.

[33] Peter Hintz and András Vasy. Semilinear wave equations on asymptotically de Sitter, Kerr-de Sitter and Minkowski spacetimes. Preprint, arXiv:1306.4705, 2013.

[34] Lars Hörmander. The analysis of linear partial differential operators. I-IV. Classics in Mathematics. Springer, Berlin, 2007.

[35] Sergiu Klainerman. Global existence for nonlinear wave equations. Comm. Pure Appl. Math., 33(1):43-101, 1980.

[36] Sergiu Klainerman and Gustavo Ponce. Global, small amplitude solutions to nonlinear evolution equations. Comm. Pure Appl. Math., 36(1):133-141, 1983.

[37] Bernard S. Kay and Robert M. Wald. Linear stability of Schwarzschild under perturbations which are nonvanishing on the bifurcation 2-sphere. Classical Quantum Gravity, 4(4):893898, 1987.

[38] Jonathan Luk. The null condition and global existence for nonlinear wave equations on slowly rotating Kerr spacetimes. Journal Eur. Math. Soc., 15(5):1629-1700, 2013.

[39] Jeremy Marzuola, Jason Metcalfe, Daniel Tataru, and Mihai Tohaneanu. Strichartz estimates on Schwarzschild black hole backgrounds. Comm. Math. Phys., 293(1):37-83, 2010.

[40] Richard B. Melrose. The Atiyah-Patodi-Singer Index Theorem. Research Notes in Mathematics, Vol 4. Peters, 1993.

[41] Richard B. Melrose. Spectral and scattering theory for the Laplacian on asymptotically Euclidian spaces. Marcel Dekker, 1994.

[42] Richard B. Melrose, Antônio Sá Barreto, and Andras Vasy. Asymptotics of solutions of the wave equation on de Sitter-Schwarzschild space. Comm. in PDE, 39(3):512-529, 2014.

[43] Stéphane Nonnenmacher and Maciej Zworski. Decay of correlations for normally hyperbolic trapping. Preprint, arXiv:1302.4483, 2013.

[44] Stéphane Nonnenmacher and Maciej Zworski. Quantum decay rates in chaotic scattering. Acta Math., 203(2):149-233, 2009.

[45] Antônio Sá Barreto and Maciej Zworski. Distribution of resonances for spherical black holes. Math. Res. Lett., 4(1):103-121, 1997.

[46] Xavier Saint Raymond. A simple Nash-Moser implicit function theorem. Enseign. Math. (2), 35(3-4):217-226, 1989

[47] Daniel Tataru. Local decay of waves on asymptotically flat stationary space-times. Amer. J. Math., 135(2):361-401, 2013.

[48] Daniel Tataru and Mihai Tohaneanu. A local energy estimate on Kerr black hole backgrounds. Int. Math. Res. Not. IMRN, (2):248-292, 2011. 
[49] Michael E. Taylor. Partial Differential Equations I-III. Springer-Verlag, 1996.

[50] Mihai Tohaneanu. Strichartz estimates on Kerr black hole backgrounds. Trans. Amer. Math. Soc., 364(2):689-702, 2012.

[51] András Vasy. Microlocal analysis of asymptotically hyperbolic and Kerr-de Sitter spaces (with an appendix by Semyon Dyatlov). Inventiones mathematicae, pages 1-133, 2013.

[52] Robert M. Wald. Note on the stability of the Schwarzschild metric. J. Math. Phys., 20(6):1056-1058, 1979.

[53] Jared Wunsch and Maciej Zworski. Resolvent estimates for normally hyperbolic trapped sets. Annales Henri Poincaré, 12:1349-1385, 2011.

[54] Shijun Yoshida and Nami Uchikata and Toshifumi Futamase. Quasinormal modes of Kerr-de Sitter black holes. Phys. Rev. D, 81(4):044005, 2010.

Department of Mathematics, Stanford University, CA 94305-2125, USA

E-mail address: phintz@math.stanford.edu

E-mail address: andras@math.stanford.edu 